\title{
Cross-Pathway Control of the Pathogenic Fungus Aspergillus fumigatus: a Manifold Stress Response System
}

\author{
Dissertation \\ zur Erlangung des Doktorgrades \\ der Mathematisch-Naturwissenschaftlichen Fakultäten \\ der Georg-August-Universität zu Göttingen \\ vorgelegt von \\ Christoph Sasse \\ aus Hess. Lichtenau
}

Göttingen 2008 
Die vorliegende Arbeit wurde in der Arbeitsgruppe von Prof. Dr. Gerhard H. Braus in der Abteilung Molekulare Mikrobiologie des Institutes für Mikrobiologie und Genetik der GeorgAugust-Universität Göttingen angefertigt.

Teile dieser Arbeit wurden veröffentlicht in:

Sasse, C., Bignell, E.M., Hasenberg, M., Haynes, K., Gunzer, M., Braus, G.H., and

Krappmann, S. (2008) Basal expression of the Aspergillus fumigatus transcriptional activator CpcA is sufficient to support pulmonary aspergillosis. Fungal Genet Biol

D7

Referent: PD Dr. Sven Krappmann

Korreferent: Prof. Dr. G.H. Braus

Tag der mündlichen Prüfung: 29.04.08 
Für Anna und meine Eltern 


\section{Danksagung}

Zunächst möchte ich PD Dr. Sven Krappmann danken, der mich während meiner Promotion hervorragend betreut hat und immer Zeit für Gespräche und Diskussionen hatte. Außerdem möchte ich ganz herzlich Herrn Prof. Dr. G.H. Braus danken, der es mir ermöglichte meine Doktorarbeit in seiner Abteilung anzufertigen.

Dank gilt auch Prof. Dr. A.A. Brakhage und Dr. O. Kniemeyer, Dr. K. Haynes und Dr. E. Bignell, Dr. W. Nierman und Dr. S. Kim als auch Prof. Dr. M. Gunzer und M. Hasenberg für die gute Zusammenarbeit ohne die diese Doktorarbeit nicht entstanden wäre.

Ebenfalls möchte ich mich ganz herzlich bei Karen Laubinger für die guten und aufmunternen Gespräche bedanken ebenso wie für die schöne gemeinsame Zeit im Labor 102. Auf diese Weise möchte ich auch Verena Große danken, die mir während meiner Doktorandenzeit viele Methoden gezeigt hat und das nötige Verständnis für Pilze beigebracht hat. Marc Dumkow, der immer Zeit für fachliche Gespräche hatte, möchte ich hiermit ebenfalls danken.

Besonderer Dank gilt Daniela Justa-Schuch, Britta Herzog und Dr. Lars Fichtner, die sich Zeit genommen haben diese Arbeit gegenzulesen. Dank gilt auch allen Mitarbeitern der AG Braus für die schöne und angenehme Zeit.

Zum Schluß möchte ich mich bei meiner Freundin Anna Bergamnn und bei meinen Eltern bedanken ohne deren Unterstützung diese Arbeit nicht möglich gewesen wäre.

Diese Arbeit wurde finanziell unterstützt von der Deutschen Forschungsgemeinschaft (DFG). 


\section{Contents}

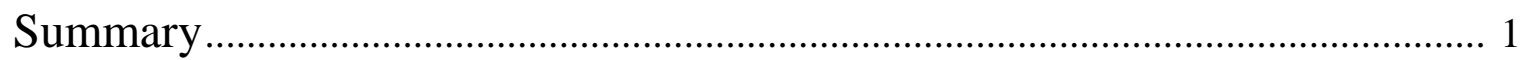

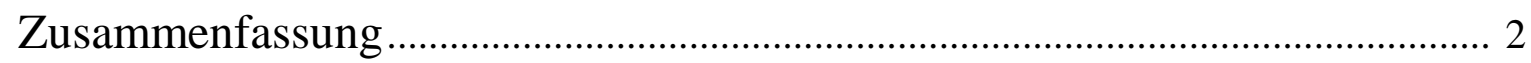

Chapter 1: Introduction ................................................................................. 4

1.1 Life and characteristics of Aspergillus fumigatus ................................................. 4

1.2 One fungus - three diseases: Aspergillus fumigatus $\&$ aspergillosis........................... 6

1.2.1 The different types of disease ............................................................. 7

1.2.2 The immune-defence-system and therapies against aspergillosis ................ 8

1.2.3 Virulence determinants of A. fumigatus ................................................. 10

1.3 The lung as environment for A. fumigatus with nutrient limitation ........................... 12

1.4 The fungal Cross-Pathway Control System ......................................................... 13

1.4.1 Induction and regulation of the CPC/GC ............................................ 14

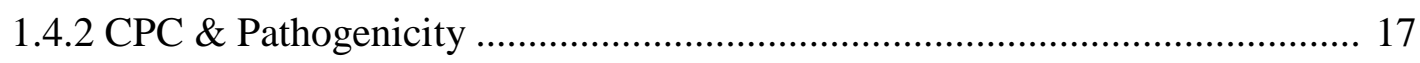

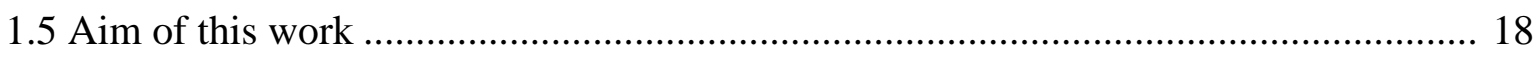

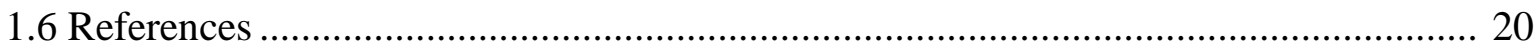

Chapter 2: CpcA, a master regulator of stress response factors ...................... 28

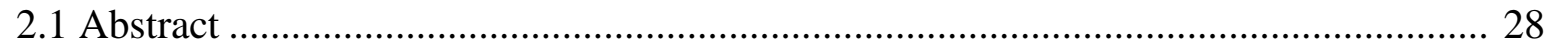

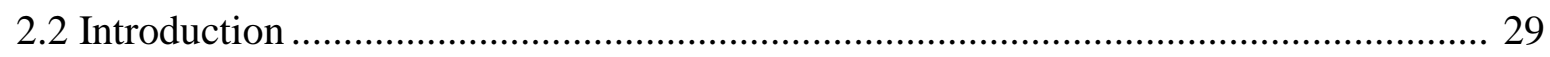

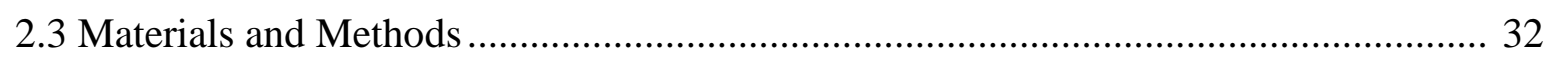

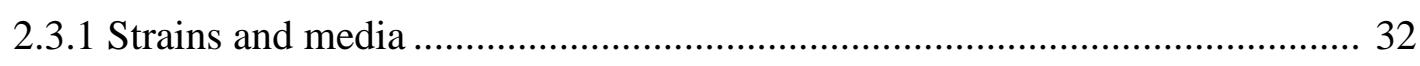

2.3.2 A. fumigatus transformation and plasmid construction .............................. 32

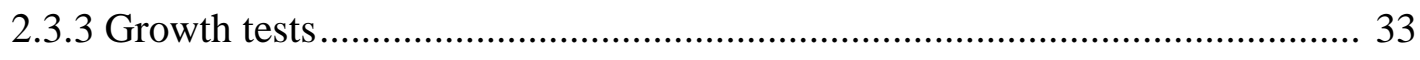

2.3.4 Transcriptome analysis and Northern hybridisation.................................. 33

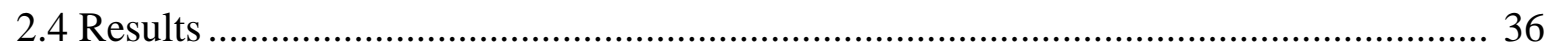

2.4.1 Transcriptional profiling of histidine-starved A. fumigatus reveals the CpcA-dependent transcriptome............................................................. 36

2.4.2 Estimation of the CpcA-dependent transcriptome..................................... 37

2.4.3 The CpcA-directed transcriptome of A. fumigatus in comparison to the transcriptional profile elicited by voriconazole treatment........................... 40 
2.4.4 CpcA-dependent stress genes

2.4.5 $c d a A$ and $c d a B$ appear to be regulated by the transcriptional activator $\mathrm{CpcA}$

2.4.6 Deletion of $c d a A$ and $c d a B$ results in increased sensitivity towards

Calcofluor White

2.4.7 Calcofluor has no inductive effect on the Cross-Pathway Control

2.5 Discussion 51

2.6 References 55

Chapter 3: Basal expression of Aspergillus fumigatus transcriptional activator $\mathrm{CpcA}$ is sufficient to support pulmonary aspergillosis. 59

3.1 Abstract 59

3.2 Introduction 60

3.3 Materials and Methods 62

3.3.1 Strains, media, and growth conditions 62

3.3.2 Transformation procedures 62

3.3.3 Manipulation of nucleic acids and plasmid constructions. 62

3.3.4 Biochemical methods and Western blots 63

3.3.5 Virulence tests in a murine model of pulmonary aspergillosis 63

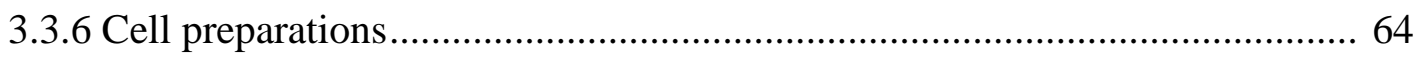

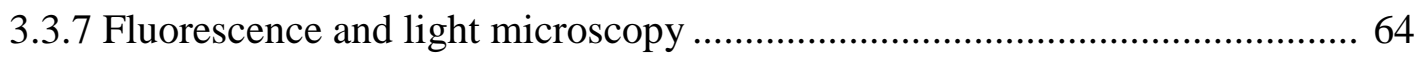

3.4 Results 66

3.4.1 Phagocytosis by macrophages does not trigger CpcA expression in Aspergillus fumigatus. 66

3.4.2 The Aspergillus fumigatus genome encodes a Gcn2p-like eIF2 $\alpha$ kinase ...... 68

3.4.3 Deletion of $c p c C$ in A. fumigatus impairs the Cross-Pathway Control response 69

3.4.4 A $c p c C$ null mutant of $A$. fumigatus is still able to phosphorylate eIF $2 \alpha \ldots \ldots .72$

3.5 Discussion 75

3.6 References 78 
Chapter 4: The basal proteome directed by the Aspergillus fumigatus

transcriptional regulator $\mathrm{CpcA}$............................................................................ 81

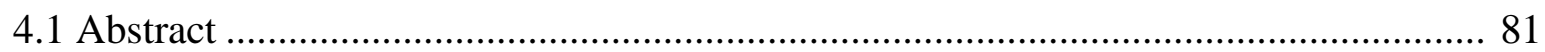

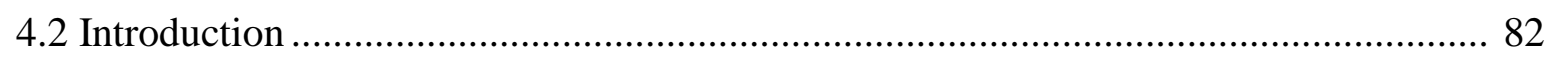

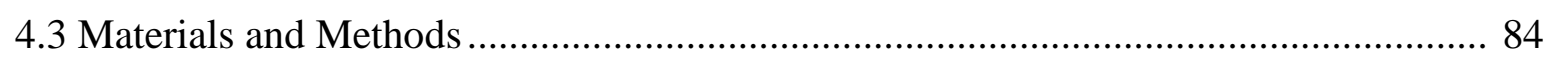

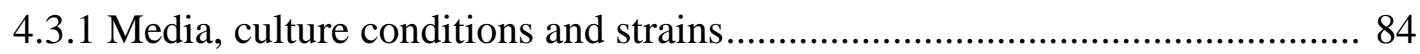

4.3.2 Protein extraction and determination of concentration .............................. 84

4.3.3 2-D gel electrophoresis................................................................................ 84

4.3.4 Visualisation of proteins by Coomassie Brilliant Blue staining (CBB)........ 85

4.3.5 DIGE experiments, analysis and identification of spots ........................... 85

4.4 Results and Discussion........................................................................................ 87

4.4.1 Differentially expressed proteins in dependency of basal CpcA expression . 87

4.4.2 Groups of function of the basal proteome directed by CpcA........................ 89

4.4.3 Presence of putative CpcA binding sites ...................................................... 92

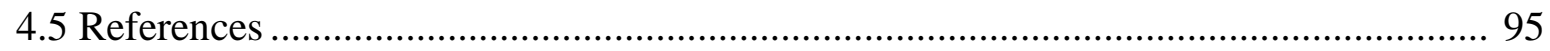

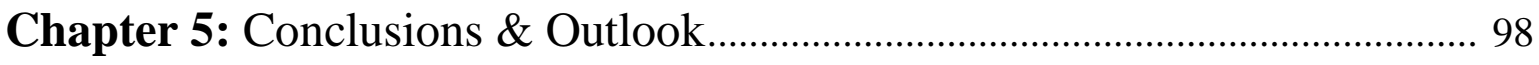

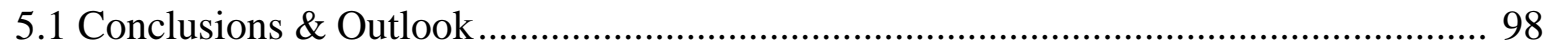

5.1.1 The CPC is a global network required for many different stress responses... 98

5.1.2 The CPC is not triggered during the beginning of infection ....................... 100

5.1.3 The basal level of $\mathrm{CpcA}$ regulates genes involved in pathogenicity.............. 101

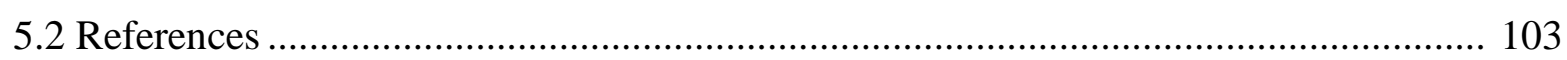




\section{Summary}

In this study the Cross-Pathway Control (CPC) of the opportunistic pathogenic fungus Aspergillus fumigatus was analysed in view of putative stress response genes, which enables the fungus to adapt within the host. Previous works displayed that the transcriptional regulator $\mathrm{CpcA}$ of the $\mathrm{CPC}$ is required for full virulence of $A$. fumigatus.

In the first part of this work an A. fumigatus wild-type and its $\Delta c p c A$ derivative were compared under conditions of amino acid starvation via transcriptome profiling. 377 genes were detected that are induced under starvation, and 146 displayed a reduced transcriptional level in comparison to the $\triangle c p c A$ deletion strain. Classification pointed out that most of the genes are involved in primary and secondary metabolism. In addition, expression of numerous genes encoding transporters and permeases was increased as well as that of some genes coding for transcriptional regulators. Further, twelve regulated genes involved in any kind of stress response like detoxification of oxidative molecules and drug resistance were found. Within these genes, two encoding so-called CpcA-dependent AAA-ATPases ( $c d a A$ and $c d a B$ ) could be identified. Deletion of these genes led to an increased sensitivity towards Calcofluor White, which is a cell wall stress-inducing drug. These results clarify the flexibility of the fungus to react on different kind of stress signals via activation of the transcriptional regulator CpcA.

The second chapter describes the CPC sensor kinase $\mathrm{CpcC}$ and its influence on pathogenicity of A. fumigatus. A $\triangle c p c C$ deletion mutant is unable to induce the expression of CpcA under amino acid starvation conditions. Moreover, the deletion strain displayed no decreased pathogenicity in a murine model of pulmonary aspergillosis. Therefore, it is suggested that the basal level of CpcA seems to be sufficient for full virulence. This finding is supported by the fact that during the early phase of infection no induction of $\mathrm{CpcA}$ expression occurs as validated by a GFP-CpcA reporter strain.

Resulting from these data a deeper insight into the proteome depending on the basal expression level of $\mathrm{CpcA}$ was sought. Therefore, DIGE experiments were performed to compare the wild-type with the $\triangle c p c A$ strain under non-starvation conditions, which resulted in identification of 22 different proteins. 14 of them displayed an increased level in the wildtype in comparison to the deletion strain. Functional classification pointed out that three of the up-regulated proteins might play a role in pathogenicity; in addition, one protein was identified that appears to be required for detoxification of reactive oxidative species.

Accordingly, this study characterises the Cross-Pathway Control system of A. fumigatus as a wide-domain regulatory system of this human pathogen. 


\section{Zusammenfassung}

In dieser Arbeit wurde die Cross-Pathway Control (CPC) des opportunistisch pathogenen Pilzes A. fumigatus im Hinblick auf mögliche Faktoren untersucht, die eine Anpassung des Organismus innerhalb des Wirtes ermöglichen. Aus vorherigen Studien war bereits bekannt, dass der transkriptionelle Regulator CpcA dieses Systems Einfluss auf die Virulenz hat. Im ersten Teil dieser Arbeit wurden mittels Transkriptomanalyse der Wildtyp und eine $c p c A$ Deletionsmutante unter Aminosäuremangelbedingungen miteinander verglichen. Dabei wurden 377 Gene identifiziert, deren Expression unter diesen Bedingungen induziert wurde, während die Expression von 146 Genen reprimiert wurde. Die Klassifizierung dieser Gene zeigte, dass die Mehrheit in Wege des primären und sekundären Metabolismus involviert ist. Darüber hinaus wurden eine Vielzahl an Transportern und Permeasen sowie Transkriptionsfaktoren gefunden, als auch ein Dutzend relevanter Gene bzgl. Stressantworten wie z. B. der Neutralisierung oxidativer Moleküle oder Resistenzmechanismen. Unter anderem wurden dabei zwei sog. AAA-ATPasen entdeckt, die aufgrund der Tatsache, dass sie Teil des CpcA-abhängigen Transkriptoms sind, als $c d a A$ und $c d a B$ bezeichnet wurden. Entsprechende Mutantenstämme zeigten als Phänotyp eine ausgeprägte Sensitivität gegenüber dem Zellwandstress induzierenden Wirkstoff Calcofluor White. Diese Ergebnisse zeigen schließlich die Anpassungsfähigkeit des Pilzes auf unterschiedliche Stresseinflüsse mittels des transkriptionellen Regulators CpcA.

Das zweite Projekt beinhaltete die Untersuchung der Sensor Kinase CpcC und deren Einfluss auf die Pathogenität von A. fumigatus. Die hierfür konstruierte Mutante zeigte unter Mangelbedingungen nur eine begrenzte Induktion von CpcA im Vergleich zum Wildtyp, was zu einem verminderten Wachstum unter Aminosäuremangelbedingungen führte. Trotz dieser geringeren Menge an CpcA zeigte der $\mathrm{CpcC}$ Deletionsstamm keine verringerte Virulenz im Mausmodel. Daraus wurde die Schlussfolgerung gezogen, daß die basale Expressionsrate von CpcA ausreichend für die Pathogenität von A. fumigatus ist. Dies wird durch die Tatsache unterstützt, dass während der frühen Infektionsphase keine Induktion der CpcA-Expression stattfindet, wie mit Hilfe eines gfp::cpcA Reporterstammes festgestellt werden konnte.

Entsprechend dieser Ergebnisse war es das Ziel, das vom basalen Expressionslevel abhängige Proteome zu charakterisieren. Dieses wurde mittels DIGE-Experimenten untersucht, was zur Identifizierung von 22 Proteinen führte, von denen 14 in einer $\Delta c p c A$-Mutante eine verminderte Expression aufwiesen. Die weitere Klassifizierung in Funktionsgruppen zeigte, dass drei dieser 14 Proteine gegebenenfalls eine Rolle für die Pathogenität von A. fumigatus 
spielen. Des Weiteren wurde ein Kandidatenprotein gefunden, dessen Funktion in der Neutralisierung von oxidativen Substanzen liegt.

Diese Arbeiten charakterisieren dementsprechend das Netzwerk der Cross-Pathway Control des Humanpathogens A. fumigatus als umfassendes regulatorisches System. 


\section{Chapter 1}

\section{Introduction}

\subsection{Life and characteristics of Aspergillus fumigatus}

The filamentous fungus Aspergillus fumigatus belongs to the phylogenetic group of the Deuteromycota, a subgroup of the Euascomycota. As a saprophytic microorganism it is able to colonise and grow on decomposing substrates, making the soil and places like hay or compost its natural habitat where it plays an important role in recycling nitrogen and carbon (Debeaupuis et al., 1997; Mullins et al., 1976). To grow on these materials, A. fumigatus secretes degrading enzymes to digest them into oligomeric or monomeric units, which then can be taken up by the fungal cell.

As it is typical for a Deuteromycete, a sexual cycle has not been described yet for A. fumigatus. During its asexual lifecycle, A. fumigatus produces small spores as it is typical for aspergilli, which are grey-green in colour and have a size of 2 to $3 \mu \mathrm{m}$. These conidia contain each one haploid nucleus and are developed from specialised cells called phialides, which are part of the spore-producing structures, the conidiophores. These extend from socalled foot cells at right angles to the vegetative mycelium to culminate in a vesicle with a size of 20-30 $\mu \mathrm{m}$ in diameter. In general, the upper parts of aspergilli conidiophores are built up by layers of specialised cells such as metulae, phialides, and conidia (fig. 1.1), with $A$. fumigatus lacking metula cells. To spread out its hydrophobic spores into the environment, the fungus depends on air currents as it lacks active mechanisms of spore dispersal (Brakhage and Langfelder, 2002; Latgé, 1999). When a spore reaches a place rich in nutrients, germination is triggered to initiate the vegetative growth state. In this state, A. fumigatus produces a white-grey mycelium consisting of branching hyphae. These are septated and contain multiple nuclei per cell. Growth and mycelial extension is executed at the hyphal tip to form a dense netting of intertwined hyphae. After a certain time, conidiophores can be formed from this mycelium to produce new spores. These conidia are then spread into the environment and a new cycle begins (fig. 1.2). 


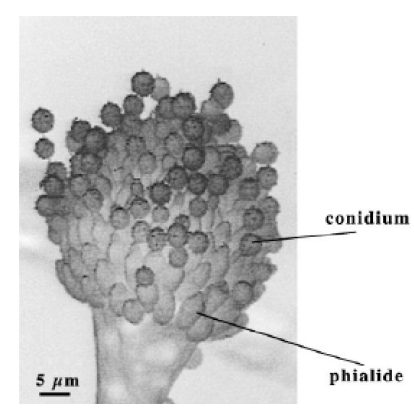

Figure 1.1. Electroscope microscopy image of a conidophore of A. fumigatus Shown is the asexual fruit body, the conidiophore, which is built up by vesicles and phialides, from which conidia are formed in chains by repeated budding (from Brakhage and Langfelder, 2002).

A. fumigatus is a fast growing fungus able to reach a colony size of $4 \mathrm{~cm}$ in a week (Raper and Fennel, 1965). Its optimal growth temperature is $37^{\circ} \mathrm{C}$, but it is able to grow at higher or lower temperatures. In general, fungi can be classified into thermophilic and thermotolerant species based on their minimum and maximum growth temperatures. In this regard, A. fumigatus is a thermotolerant fungus able to grow from under $20^{\circ} \mathrm{C}$ up to $55^{\circ} \mathrm{C}$, which is in contrast to thermophilic ones with a growth temperature minimum at or above $20^{\circ} \mathrm{C}$ and a maximum at or above $50^{\circ} \mathrm{C}$. This is an important characteristic that distinguishes A. fumigatus from other aspergilli like A. flavus, A. niger, or A. terreus (Chang et al., 2004; Cooney and Emerson, 1964; Maheshwari et al., 2000).

As mentioned above, A. fumigatus lacks an obvious sexual cycle. However, in earlier studies it was shown that two different mating-types exist for this Deuteromycete, which leads to the suggestion that a sexual cycle is possible (Dyer and Paoletti, 2005). It is known that parasexuality, which is typical for asexual fungi and enables genetic recombination to a certain degree, is present in A. fumigatus. The first step of the parasexual cycle is that different A. fumigatus strains grow next to each other, followed by hyphal fusion events and plasmogamy to form a stabilised heterokaryon containing nuclei of both parental strains. Within this, infrequent diploidisation and haploidisation events result in chromosome exchanges and therefore recombination of the genetic contents (fig. 1.2). As a result, a mycelium is formed that enables the fungus to develop a selective advantage in its natural environment (Berg and Garber, 1962; Stroemnaes and Garber, 1962).

With the completed sequencing of A. fumigatus in 2005 the research on this organism became easier resulting in a better understanding of this filamentous fungus. On eight chromosomes 9,926 putative protein encoding genes were found with a total size of $29.4 \mathrm{Mb}$. Nearly a third of the predicted genes are of unknown function (Nierman et al., 2005; Ronning et al., 2005). Thus more time will be required for a final annotation of the A. fumigatus genome. 


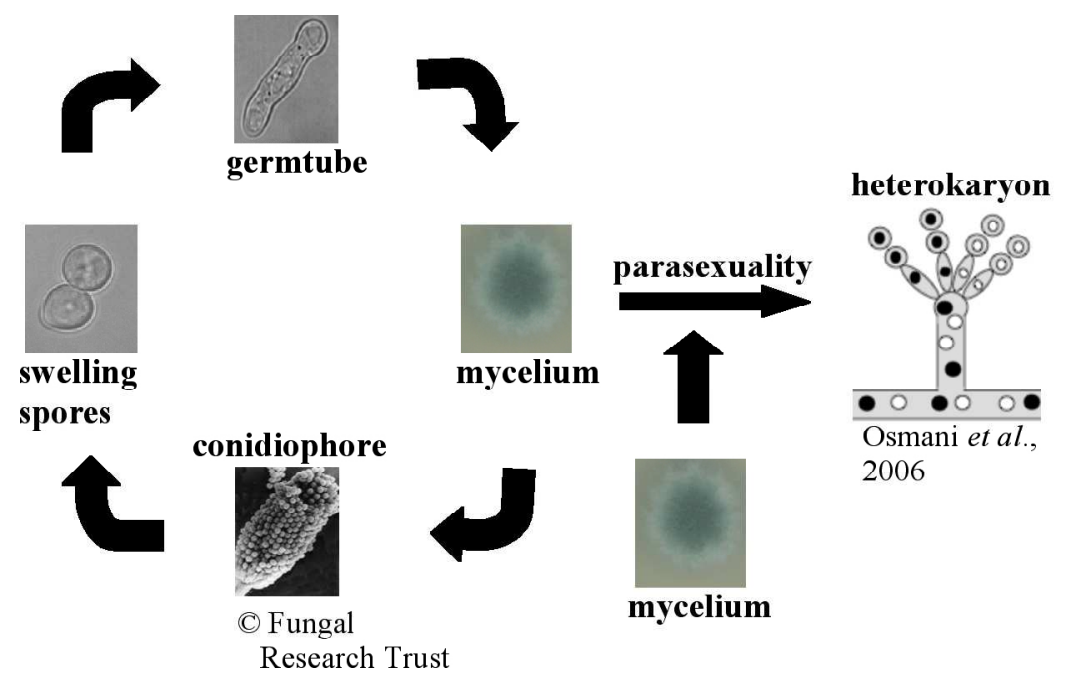

Figure 1.2. Lifecycle of the filamentous fungus $A$. fumigatus

The scheme displays the asexual cycle of $A$. fumigatus with its possibility for parasexuality with a different strain resulting in a stabilised heterokaryon.

\subsection{One fungus - three diseases: Aspergillus fumigatus \& aspergillosis}

As outlined above, A. fumigatus belongs to the group of saprophytic, filamentous fungi dwelling the soil as a natural habitat. Moreover, this fungus can also be an opportunistic pathogen for humans. In the last two decades, A. fumigatus has become one of the most dangerous fungal pathogens for immunocompromised individuals, with the risk of acquiring a disease termed aspergillosis that is usually localised in the respiratory tract. The primary site of entry is the host's lung, although infection by A. fumigatus is not limited to this location as it can colonise the skin or disseminate to other organs like kidneys and liver (Denning, 1998; Dixon and Walsh, 1992; Kwon-Chung and Bennett, 1992; Latgé, 1999; Lortholary et al., 1995; Prescott et al., 1992). Primarily, infection results from inhalation of the airborne spores, which are small enough to reach the aveoli of the respiratory system. Most patients suffering from aspergillosis have an impaired immune system that is often evoked by leukaemia, neutropenia or after prolonged treatment with steroids, such as solid organ transplantation patients. The mortality rate of aspergillosis among these patients lies between 30 to $90 \%$ (Ellis, 1999; Latgé, 1999). To a limited extent, immunocompetent persons can acquire aspergillosis, but the number of incidences is by far not as high as compared to the one among immunocompromised patients (Ellis, 1999). 


\subsubsection{The different types of disease}

Aspergillosis can be divided in three different forms of disease: allergic bronchopulmonary aspergillosis (ABPA), the so-called aspergilloma, and invasive forms of aspergillosis (IA) (fig. 1.3).

$\mathrm{ABPA}$ is one of the most severe allergic pulmonary disorders that is induced by aspergilli. It resembles classic asthma with an immune reaction of T-cells against the fungus (Latgé, 1999; Murali et al., 1997; Patterson et al., 1986). This kind of aspergillosis is predominantly found in patients suffering from asthma or cystic fibrosis and may result in a fatal destruction of the lung (Patterson et al., 1982; Rosenberg et al., 1977). In weaker forms it appears as an asthmatic illness without severe damage of the respiratory tract. One major problem is still to diagnose this kind of disease and to distinguish between usual forms of asthma where no fungus is involved and the Aspergillus-depended form. The standard diagnostic techniques differ from patient to patient as well as from the status of the disease. Means of ABPA diagnosis are still limited, which often results in undetectable disease leading to the concept of "silent" ABPA (Schönheyder et al., 1988). In most cases, patients having an ABPA have to be treated with antifungal drugs; in the worst case scenario, untreated patients may die by the failure of the respiratory system (Latgé, 1999).

The second form of aspergillosis is the so called aspergilloma, often referred to as "fungus ball" (Shibuya et al., 2006). Typical patients are persons having a cystic disease of the lung or a tuberculosis infection. The aspergilloma is usually a limited ball of mycelium without invasive growth. In some cases the non-invasive proliferation of hyphae turns into an invasive form of aspergillosis, e.g. when the immune system is impaired by suppressive therapies or by some kind of illness (Shibuya et al., 2006). In immunocompetent persons the fungus has not the ability to grow invasively, a dissemination of invasive aspergillosis is therefore unlikely. The third type of aspergillosis is the so-called invasive type (IA), which is the most severe form of disease evoked by this fungus. Mortality rates in this case range from 30 to $90 \%$, and this disease is characterised by invasive fungal growth within the host. IA can be sub-divided in four types which are (Denning, 1998; Hope et al., 2005; Verweij and Denning, 1997): 1.) acute or chronic pulmonary aspergillosis (Latgé, 1999); 2.) tracheobronchitis and obstructive bronchial disease with various degrees of invasion of the mucosa and cartilage as well as pseudomembrane formation, as it is predominantly found in AIDS patients (Denning et al., 1991; Kemper et al., 1993; Nash et al., 1997); 3.) acute invasive rhinosinusitis (Drakos et al., 1993; Morgan et al., 1984; Savage et al., 1997; Viollier et al., 1986; Washburn and Bennett et al., 1988); 4.) disseminated disease founding in the brain and other organs of the host (Bodey 
et al., 1992; Pagano et al., 1996; Ribaud et al., 1999; Wingard et al., 1987). In the early state of IA it is very difficult to diagnose an infection by common techniques like CT scan or microscopy. Symptoms giving a hint for an invasive aspergillosis are often too unspecific. Therefore, an improved diagnostic regime is needed in order to detect the different forms of IAs (Latgé, 1999).

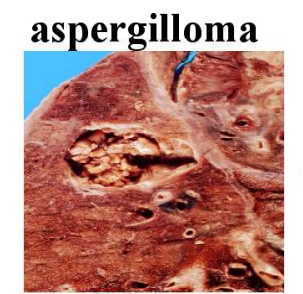

pathhsw5m54.ucsf.edu case16/aspergilloma.html

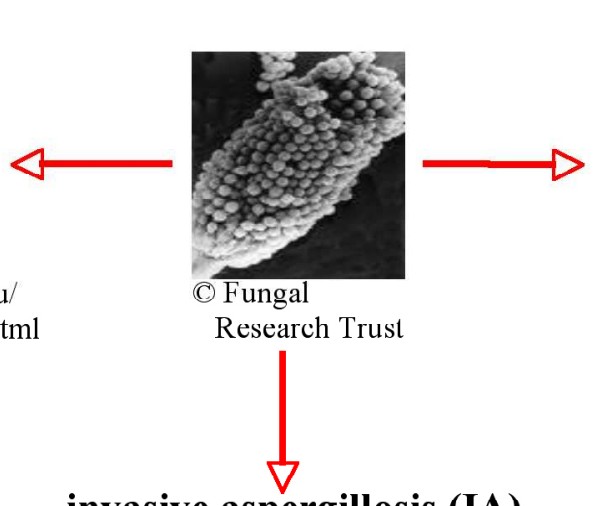

invasive aspergillosis (IA)

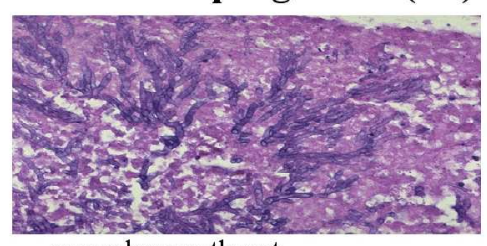

www.lungpath.net

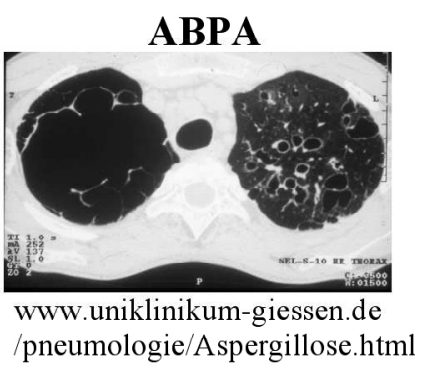

/pneumologie/Aspergillose.html

Figure 1.3. The three forms of aspergillosis

The figure displays the three typical types of aspergillosis evoked by different aspergilli like A. fumigatus. The aspergilloma and the ABPA (allergic bronchopulmonary aspergillosis) are in general no invasive forms, in contrast to the IA, the worst form of aspergillosis.

\subsubsection{The immune-defence-system and therapies against aspergillosis}

Invasive forms of aspergillosis normally appear in persons, whose immune system is suppressed by some kind of illness such as leukaemia or by treatment with pharmaceuticals after organ transplantations. In a functional immune system there are different defence mechanisms to react on invasive growth of parasitical organisms. One important group are cells that have the ability for phagocytosis (Latgé, 1999). On one hand there are macrophages, which are the first defence barrier of the host against the fungus. These cells have the ability to bind via lectin-like interactions to a special receptor, which can be found on the cell wall of the conidia (Kan and Bennett, 1988, 1991; Serrano-Gomez et al., 2004). After absorption, the spore resides in the phagosome where lysis starts. The dying process of the spores in macrophages is slow, so that after 24 hours still $10 \%$ of the ingested spores survive (Schaffner, 1985; Schaffner, 1992). The second phagocytosing barrier is constituted by neutrophils. These are specialised on germinating spores and single hyphae emerging from them. Mechanisms for killing spores are based on destroying the fungal cell wall. For 
recognition of the germinating conidia neutrophils detect the same cell wall protein as macrophages do (Serrano-Gomez et al., 2004). Killing by neutrophils happens fast, so that after 2 hours $50 \%$ of the phagocytised spores are eliminated (Roilides et al., 1991). Additionally, T-cells are activated and seem to be an important defence against invasion (Hohl and Feldmesser, 2007). In an immune-suppressed individual with impaired function or absence of macrophages and/or neutrophils, fungal spores will not be attacked and can germinate without any restriction by the host. As also the mycelium cannot be attacked, the fungus gets the ability to grow invasively and may reach the blood stream from which other parts and organs of the host can be infected.

To date, there are four drugs that are used generally against aspergillosis (Espinel-Ingroff et $a l ., 2005)$. One of them is amphotericin B (amB), still the "gold standard" against this disease evoked by A. fumigatus. This drug binds to membrane sterols resulting in the formation of membrane channels, which increase the cell's permeability. Moreover, it inhibits the activity of proton ATPase pumps. There are two main disadvantages of amphotericin B: one is its insolubility in water and therefore difficulties in applying the drug to the host at sufficient doses; the other point is its toxicity, based on the fact that amphotericin B, although being a reagent that targets ergosterol, displays a pronounced affinity to the cholesterol of mammalian cells, resulting in their destruction. Nevertheless, to date amphotericin B is the most effective antifungal drug against A. fumigatus (Bolard, 1986; Brajtburg and Bollard, 1996; Clements and Peacock, 1990; Meletiadis et al., 2007; Pathak et al., 1998; Patterson, 1998). Other common anti-fungal drugs are itraconazole, voriconazole and posaconazole, which belong to the triazole family. The effect of the triazoles in general lies in inhibition of ergosterol biosynthesis. Itraconazole, for example, inhibits the enzyme cytochrome P-450 14 $\alpha$ demethylase, which is important for ergosterol production. Reduced levels of ergosterol increase membrane fluidities and thus result in increased permeability. One of the advantages when using triazoles as antifungal drug is that these are very specific for fungi and have less effect on mammalian cholesterol biosynthesis. Thus, there are less adverse effects in IA patients and the compatibility is higher as it is with amphotericin B. However, triazolecontaining drugs bear some disadvantages: It was shown that some A.fumigatus strains acquires resistance especially against itraconazole; also an i.v. preparation for itraconazole is not available (Chryssanthou, 1997; Denning et al., 1997; Denning et al., 1997; Latgé, 1999). This problem does not exist for other triazoles like voriconazole, which have also other advantages in comparison to itraconazole (Pfaller et al., 2002). A common drawback is the high amount of required drug, but the concentration shows no strong adverse effects in 
patients with invasive aspergillosis. Nevertheless, all of these used triazoles show less effect against $A$. fumigatus than amphotericin B and are mostly fungistatic in contrast to amB, which is fungicidal. The effects of the described antifungal drugs are typical for A. fumigatus and may differ for different species of aspergilli such as A. flavus or A. terreus (Meletiadis et al., 2007). In general it has to be stated that the palette of different antifungal drugs is still limited and new appendages of therapies and pharmaceuticals have urgently to be found.

\subsubsection{Virulence determinants of $A$. fumigatus}

To invade the host and overcome any residual immune system, A. fumigatus needs special abilities (Latgé et al., 1997; Latgé et al., 1994). It must be able to survive in a nutrient-limited area for which specific biosynthetic pathways might be necessary. One important point to initiate growth inside the host is the production of adhesins, which enable A. fumigatus to adhere at the respiratory epithelia. Thereby the conidia are able to bind with their cell surface on membrane-associated proteins. These interactions between the spores' cell wall and host proteins can happen both in a specific or unspecific manner. Typical binding targets for the fungus are fibrinogen, laminin, fibronectin, and type IV collagen (Bromley and Donaldson, 1996; Gil et al., 1996; Penalver et al., 1996; Tronchin et al., 1993). The interacting compounds and molecules for adhesion to proteins of the host epithelium, which can be e.g. carbohydrates, protein molecules and glycoproteins, are localised in the inner and outer cell wall layer. To bind on hydrophobic proteins, A. fumigatus contains so-called hydrophobins that are located in the surface layer of dry conidia and can also been found in other fungal spores. Although all these adhesins appear important for fungal adhesion in the host, their role in or contribution to pathogenicity is still unclear (Latgé, 1999; Thau et al., 1994).

Conidial pigmentation, resulting in the typical grey-green colour, seems to be important for fungal resistance. White spores have a more permeable cell wall and are more susceptible to antifungal drugs compared to wild-type conidia. An important compound in pigmentation is melanin, which likely enables the fungus to counteract the immune defence system and increases spore resistance (Hogan et al., 1996; Latgé, 1999; Tsai et al., 1997; Verweij et al., 1998). Deletion of the $p k s P$ laccase results in white-coloured spores, which are reduced pathogenicity compared with the wild-type (Jahn et al., 1997; Jahn et al., 2000; Langfelder et al., 1998; Langfelder et al., 2001; Tsai et al., 1998). In contrast to this leads the deletion of the abr2 gene, which encods also a laccase in A. fumigatus, not to a reduction of pathogenicity (Sugareva et al., 2006). To summarize it can be said that proteins involved in pigmentation are not necessarily required for virulence. 
Other putative virulence factors of A. fumigatus are toxic molecules, which are often products of secondary metabolism. Gliotoxin is one of the best-studied toxins of A. fumigatus. It inhibits the phagocytosis by macrophages and can induce their apoptosis. This effect could also be seen for polymorphonuclear leucocytes (PMN) but in a reduced form (Eichner et al., 1986; Kamei and Watanabe, 2005; Orciuolo et al., 2007; Waring et al., 1988). Other molecules with a toxic effect are the 18-kDA RNase and a hemolysin (Asp-HS). The RNase has the ability to digest the $28 \mathrm{~S}$ rRNA of eukaryotes. The protein is secreted into the environment and can kill cells that are in the vicinity of the spore. In IA patients this secrete can be found in the urine (Arruda et al., 1992a; Arruda et al., 1992b; Kurup et al., 1994; Lamy and Davies, 1991; Latgé, 1999). The hemolysin, which enables the fungus to disrupt blood cells, contains negatively charged domains and can also be detected in infected patients. However, despite of the facts that the hemolysin has toxic effects it seems not to be a main virulence factor but a compound that increases the effects of other toxic factors involved in pathogenicity (Ebina et al., 1983; Fukuchi et al., 1996; Malicev et al., 2007; Yokota et al., 1985). An additional large group of putative virulence factors are enzymes displaying different kinds of function. Some proteases for example seem important for full virulence of A. fumigatus. They are needed to obtain nutrients by degradation of collagen and elastin, which constitute the main compounds of the lung (Monod et al., 1995). In further works it could be shown that clinical isolates have a higher production of proteases than the environmental pendants, and some of these enzymes lead to a detachment of epithelium cells in the respiratory tract. The major protease under neutral conditions is the serine alkaline protease $A L P$ with the ability to proteolyse elastin. Nevertheless, strains without $A L P$ cause the same mortality rate in a mouse model compared to those with $A L P$. Other enzymes without proteolytic activity are also important for A. fumigatus in causing disease (Grant, 2001; Smith et al., 1994; Tang et al., 1992; Tang et al., 1993; Tekaia and Latgé, 2005): catalases and peroxidases are needed by the organism to counterattack reactive oxidative species like $\mathrm{H}_{2} \mathrm{O}_{2}$ (Shibuya et al., 2006). Deletion of four enzymes which are involved in peroxide breakdown lead to strains which are more sensitive to this reactive oxidative molecule but not to phagocytosis (Paris et al., 2003). Thus, the role of catalases and peroxidases for A. fumigatus pathogenicity has still to be proven.

In general it has to be stated that pathogenicity of $A$. fumigatus appears to be multifactorial and cannot be assigned to one single trait (d'Enfert et al., 1996; Latgé, 1999; Tekaia and Latgé, 2005). 


\subsection{The lung as an environment for $A$. fumigatus with nutrient limitation}

As described before, A. fumigatus is a typical saprophyte that can degrade decaying material in order to gain nutrients from the environment (Debeaupuis et al., 1997; Mullins et al., 1976). When spores of this fungus colonise the lung of the host, nutrients are likely to be limited. Nitrogen and carbon sources may not be as easy available as it is the case in the natural environment. Therefore, the fungus depends on special metabolic pathways that support its growth under depleted conditions. For other pathogenic organisms it was shown that these kinds of pathways are often involved in pathogenicity. Earlier studies pointed out that the lipid metabolism of pathogenic organisms like Candida albicans and Mycobacterium tuberculosis is essential for virulence (Bishai, 2000; Lorenz and Fink, 2001, 2002; McKinney et al., 2000; Munoz-Elias and McKinney, 2005). These organisms are able to extract carbon sources from the host via the glyoxylate cycle. The key enzyme of this pathway is an isocitrate lyase, whose deletion leads to a reduced virulence of $M$. tuberculosis and $C$. albicans (Lorenz and Fink, 2001; Munoz-Elias and McKinney, 2005). Investigations of the isocitrate lyase of A. fumigatus revealed that in this organism the glyoxylate cycle is not important for virulence as an isocitrate lyase mutant has the same virulence in murine model as the wild-type, and this lead to the suggestion that the lipid metabolism is not sufficient to exploit C-sources in the lung (Ibrahim-Granet et al., 2008; Schöbel et al., 2007). Therefore, A. fumigatus uses other pathways and enzymes to metabolise nutrients from the environment. The lung of mammals, consisting mostly of proteins like collagen, is a habitat where nutrients are unbalanced. For degradation of these tissues the fungus needs special enzymes like proteases which results in mobilisation of amino acids, which can be used as sources of nitrogen and carbon (Kogan et al., 2004). Free amino acids are absorbed and metabolised via different pathways to support fungal growth. An important route in carbon metabolism is the methylcitrate cycle: the amino acids isoleucine, valine and methionine are converted to propionyl CoA, which then can be channelled into this patway. Deletion of the methylcitrate synthase, a key enzyme of this metabolic route, results in reduced fungal virulence. One possible explanation for this is that propionyl CoA cannot be metabolised and accumulates in the fungus where it has a toxic effect (Brock and Buckel, 2004; Ibrahim-Granet et al., 2008; Maerker et al., 2005; Zhang and Keller, 2004). As a result growth in the host lung is reduced. Other metabolic pathways appear to be also important for A. fumigatus during invasive growth. Primary metabolism pathways involved in producing substances like paraaminobenzoic acid, pyrimidine, or lysine are also required for the growth in the host and therefore for full virulence. In addition to carbon and nitrogen, the fungus also needs trace 
elements such as iron. In contrast to many other pathogens, A. fumigatus does not contain a specific mechanism for utilization of host iron sources (Ramanan and Wang, 2000; Ratledge and Dover, 2000; Schrettl et al., 2004). The fungus uses two systems for iron uptake: the reductive iron assimilation system and the siderophore-assisted iron mobilization system (Haas, 2003; Leong and Winkelmann, 1998; Van Ho et al., 2002). In earlier works it was demonstated that the sidA gene, which is involved in biosynthesis of an A.fumigatus siderophore, is important for growth in the host. In contrast to this, the ftrA gene encoding an iron permease that is involved in the iron assimilation system seems not to be important for survival in the host (Schrettl et al., 2004).

To react on limited and changing conditions of the environment, the fungus needs special sensors and regulatory proteins by which uptake systems and metabolic pathways are induced. Changing conditions of glucose for example are detected by the cAMP/protein kinase (Ruijter and Visser 1997). The nitrogen assimilation under poor conditions is regulated by different kind of proteins like the areA gene product, the Ras-related protein RhbA, or CpcA, the regulator of the Cross Pathway Control (CPC) system of amino acid biosynthesis (Hensel et al., 1998; Krappmann et al., 2004; Panepinto et al., 2002; Panepinto et al., 2003). Deletion of the areA gene leads to delayed invasive growth in the host resulting from the disability to take up certain nitrogen sources. Strains without the $r h b A$ gene are also attenuated in virulence like a $c p c A$ deletion strains. Integral to the CPC is an eIF2 kinase, which is the sensory element of this system. It can react on different kinds of limitations like amino acid starvation and stress conditions perceived from the environment (Natarajan et al., 2001) of this sensor kinase resulting in increased levels of the transcriptional activator CpcA that in turn activates genes important for different kind of metabolic pathways, among them cellular amino acid biosynthesis.

\subsection{The Fungal Cross-Pathway Control System}

Microorganisms have to be able to react to changing environmental conditions. In order to have a higher chance of survival under rapidly evolving environmental situations, they must have a system that is sensitive and that enables them to react on these ascendancies. Such kinds of regulatory systems were detected in various fungi like the yeast Saccharomyces cerevisisae (Hinnebusch, 1984) or in filamentous moulds like Neurospora crassa and Aspergillus nidulans (Carsiotis et al., 1974; Davis, 2000; Piotrowska, 1980; Sachs, 1996) where they were named "General Control of amino acid biosynthesis" (GC) or "CrossPathway Control" (CPC), respectively. They are activated under amino acid starvation 
conditions and result in a regulation of different sets of genes involved in amino acid biosynthesis, purine biosynthesis, nitrogen and sulfur metabolism and vitamin biosynthesis (Hinnebusch, 1997; Mirande, 1988; Mösch et al., 1991; Natarajan et al., 2001; Tian et al., 2007).

In its core, this signal transduction system consists of a sensor kinase, which is able to phosphorylate a subunit of the eukaryotic translation initiation factor eIF2, and a transcriptional activator that serves as downstream effector to generate a cellular read-out.

\subsubsection{Induction and regulation of the $\mathrm{CPC} / \mathrm{GC}$}

The regulatory gene of the yeast General Control was named GCN4 (general control nonderepressible), and its mRNA contains four small coding sequences, which are part of an unusually long leader region preceding the actual coding sequence. The Aspergillus gene homologous to GCN4 is $c p c A$. The $c p c A$ gene of $A$. fumigatus has a size about $2.2 \mathrm{~kb}$, from which the coding sequence spans $810 \mathrm{bp}$. Two small ORFs exist preceding the $c p c A$ coding region, which are similar to the four regulatory uORFS of GCN4 (fig. 1.4).

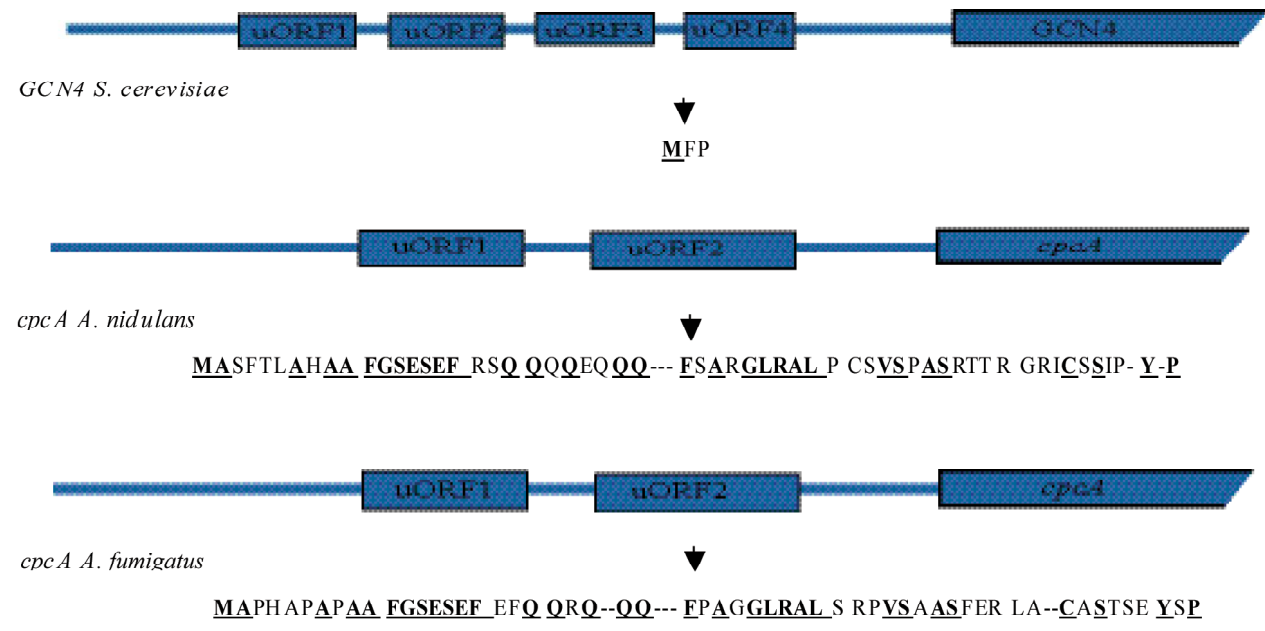

Figure 1.4. Comparison of the cpcA in A. fumigatus with the GCN4 of S. cerevisiae and the cpcA of A. nidulans. The figure shows the single uORFs of $c P c A$ and GCN4 respectively. The amino acid sequence of the $4^{\text {th }} \mathrm{uORF}$ of $S$. cerevisiae and the $2^{\text {nd }}$ uORFs of A. nidulans and A. fumigatus is written below the corresponding gene. Homologous parts of the sequences are marked bold and underlined.

The relationship between structure and function of the GCN4 gene product has been investigated in detail. The C-terminal part contains a leucine-zipper motif that is important for the dimerisation of the protein. Furthermore, a conserved DNA binding region can be found in the C-terminal domain, which is important for transcriptional regulation of Gcn $4 p$ target genes (Ellenberger et al., 1992). The N-terminal region is divided in two parts that represent 
different transcriptional activation domains (Drysdale et al., 1998). All these elements are conserved in the A. fumigatus cpcA gene product as estimated from the deduced amino acid sequence. The CpcA protein consists of 252 amino acids and has a calculated molecular weight of about $27 \mathrm{kDa}$. The activation of other genes by $\mathrm{CpcA}$ is affected by the binding at the promoter regions of these genes resulting in increased levels of transcription.

Regulation of $c p c A$ expression in the related fungus $A$. nidulans is mediated on the transcriptional as well as the translational level (Hoffmann et al., 2001), and it is likely that regulation of $A$. fumigatus $c p c A$ resembles this pattern. In contrast to this, expression of GCN4 in S. cerevisiae is mainly regulated on translational level, whereas in C. albicans transcription of GCN4 is more important than translation (Tournu et al., 2005). In detail, translational regulation of gene expression via the upstream open reading frames of GCN4/cpcA transcripts acts like follows: under sated conditions, expression levels are low at a basal level due to the fact that scanning ribosomes initiate translation on the $G C N 4 / c p c A$ transcript at the first uORF. Important for this are the amounts of so-called tertiary complexes, which consist of the translation initiator eIF2, GTP and a tRNA ${ }^{\mathrm{Met}}$, and these three components are crucial factors for initiation of translation. The tertiary complex enables the ribosomes to bind at the first uORF within the leader sequence of $G C N 4 / c p c A$. After reaching the stop codon, ribosomes disassemble into the two ribosomal sub-domains. To initiate another round of translation, a new tertiary complex has to be assembled together with the small ribosomal subunit. In case concentrations of tertiary complexes are high, one of the downstream uORFs will be translated. As the distance between the stop codon of the distal uORF and the start codon of the actual coding sequence is too close translational re-initiation is hampered and almost no gene product is expressed. Under starvation conditions expression is drastically de-repressed. In case of amino acid starvation, uncharged tRNA molecules accumulate and bind to a sensor kinase called $\mathrm{Gcn} 2 \mathrm{p}$ or $\mathrm{CpcC}$, respectively. The activated kinase phosphorylates a subunit of the initiation factor eIF2, resulting in diminished levels of tertiary complexes and therefore lower rates of translation initiation. In consequence, overall cellular translation is down-regulated but translation initiation at the $c p c A / G C N 4$ coding region increases, as competent ribosomes that have failed to translated the distal uORF are now able to bind at the start codon of the coding sequence to initiate the translation (fig. 1.5) (Braus et al., 2004). 
non-starvation conditions

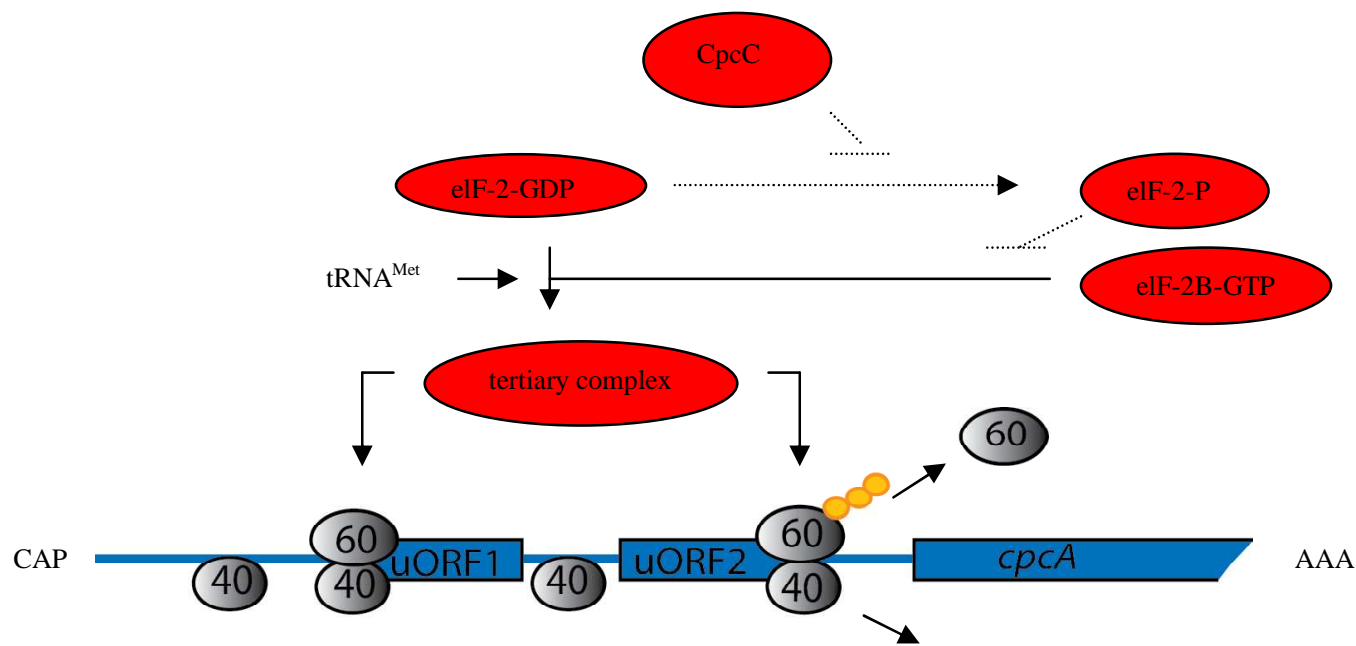

(40)

starvation conditions

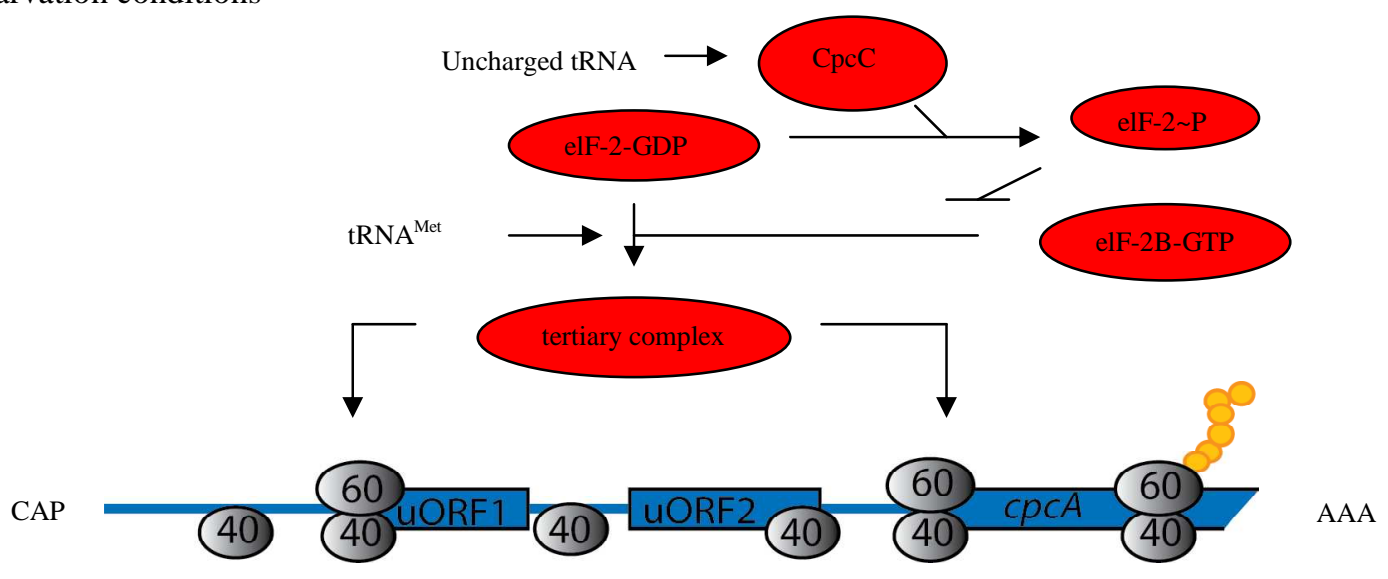

Figure 1.5. Regulation of translation of $c p c A$ in A. fumigatus

The picture shows a hypothetical model of the regulation of $c p c A$ corresponding to the yeast model. Under nonstarvation conditions sufficient amounts of charged tRNA molecules are in the cell resulting in high levels of tertiary complexes. This leads to a translation of the $1^{\text {st }}$ and $2^{\text {nd }}$ uORF but not of the coding sequence of the gene. Under starvation conditions the level of uncharged tRNAs increases, which is recognised by the sensor kinase CpcC. Activated CpcC phosphorylates eIF-2-GDP to eIF-2 P, which leads to a lower amount of tertiary complexes. Thus, the coding sequence of the $2^{\text {nd }}$ uORF cannot be translated and the scanning ribosomes bind to the start codon of the $c p c A$ coding region to initiate translation.

Regulation of Aspergillus CpcA expression on the transcriptional level has been investigated in detail for A. nidulans (Hoffmann et al., 2001). There, synthesized CpcA binds to so-called CPREs (Cross-Pathway Control Recognition Elements) that locate within the promoter region of target genes. It could be shown that two of these CPREs are part of the promoter region of cpcA suggesting a functional auto-regulatory loop (Hoffmann et al., 2001). A regulation like this has not been proven for CpcA expression in A. fumigatus; however, it was shown that two 
highly conserved CPREs are also present within the cpcA promoter region (Krappmann et al., 2004). The proximal one (5'-ATGACTCAC-3', pos. -1062 till -1053) is identical to its A. nidulans counterpart, the distal one shows a difference in one site of the sequence in comparison to the A. nidulans CPRE (5'-ATGACTCgAC-3', -1273 till -1264). This leads to the suggestion that binding of $\mathrm{CpcA}$ to this recognition site might be weaker (Arndt and Fink, 1986; Hinnebusch, 1984; Thireos et al., 1984).

In addition, a variety of other factors that influence activity of the Cross-Pathway Control/General Control activators $\mathrm{CpcA} / \mathrm{Gcn} 4 \mathrm{p}$ were identified, among them: glucose, nitrogen and purine starvation (Braus et al., 2004; Grundmann et al., 2001; Hinnebusch and Natarajan, 2002) (fig. 1.6).

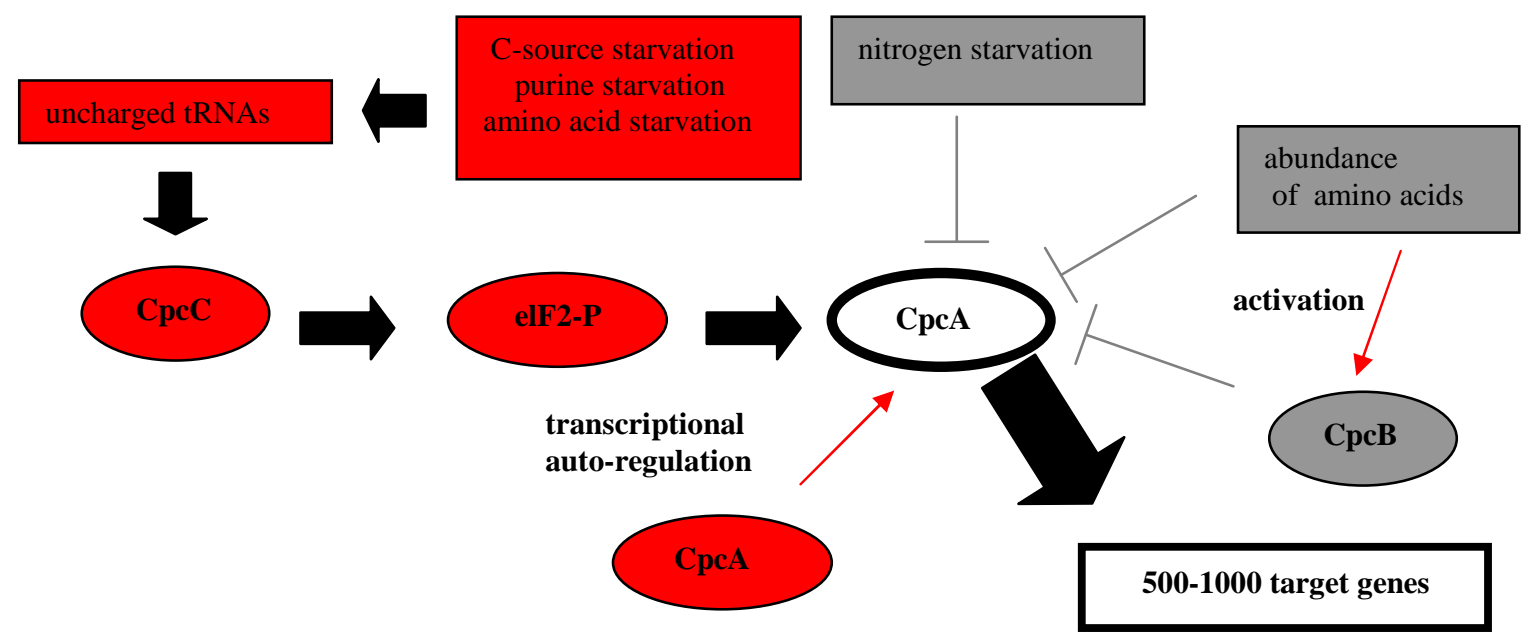

Figure 1.6. Factors with an effect on $\mathrm{CpcA}$

The scheme displays the different kinds of factors that have an effect on CpcA. The arrows show activating influences on CpcA.

\subsubsection{CPC \& Pathogenicity of A. fumigatus}

As outlined above, pathogenicity of A. fumigatus depends on several attributes, and conclusively, a plethora of genes and their products influence its virulence (Latgé, 2001). In earlier works it was demonstrated that $c p c A$ has an influence on pathogenicity of $A$. fumigatus (Krappmann et al., 2004). In this particular study, cpcA deletion mutants had been generated lacking either the coding region or the complete gene locus. Both strains showed, in comparison to the wild-type, reduced growth on minimal medium containing 5-methyl-DLtryptophan (5MT), which is a tryptophan analogue and leads to a feedback inhibition of the tryptophan biosynthesis resulting in amino acid starvation conditions. The reconstitution of both strains with $c p c A$ led to normal growth on 5MT containing medium. In histidine- 
depleted cultures, both deletion strains showed no induction of the Cross-Pathway Control, as it was shown in Northern experiments using qualified reporter genes. The influence of $c p c A$ with respect to pathogenicity was tested in a murine model of pulmonary aspergillosis using leukopenic mice. Infection of the mice with the wild-type strain resulted in a dying rate of $85 \%$ after five days. In contrast to this, dying rates among the group of mice infected with the cpcA deletion strains were at $44 \%$ and $31 \%$ after 5-6 days. Homologous reconstitution of the сpcA locus in the deletion strains restored pathogenicity to wild-type levels. Furthermore it could be shown by competitive infection in immunosuppressed mice that the wildtype had a growth advantage in comparison to either $c p c A$ deletion strain. These results demonstrate that the absence of the $c p c A$ gene product results in attenuated virulence. One possible explanation for this phenotype is nutritional limitation within the host's lung, making the Cross-Pathway Control system necessary for fungal growth and therefore virulence.

\subsection{Aim of this work}

In recent decades, A. fumigatus has become one of the most menacing pathogenic fungi for immunocompromised individuals. Factors and characteristics that enable the fungus to change from a saprophyte to a pathogen are still unclear. It was shown that special mechanisms and pathways contribute to virulence and that pathogenicity of Aspergillus is a multifactorial phenomenom (Latgé, 1999). Among these factors is the Cross-Pathway Control system effector CpcA (Krappmann et al., 2004). In this work the Cross-Pathway Control should be analysed with respect to stress resistance and pathogenicity of A. fumigatus.

The first project describes the analysis gene expression under conditions of amino acid starvation by means of microarray hybridisations with the aim to get a comprehensive overview on the CpcA-directed transcriptome of A. fumigatus. Data from other fungi had revealed that the CPC system is not restricted to conditions of amino acid starvation but may act as a general response system to act upon conditions of nutritional or environmental stress. Therefore, transcriptional profiling should evaluate the A. fumigatus CPC system with respect to stress resistance of this pathogen.

The second part of this $\mathrm{PhD}$ work deals with the eIF2 kinase $\mathrm{CpcC}$, the orthologue of yeast Gcn2p, and the role of the Cross-Pathway Control during phagocytosis by macrophages. For that purpose a suitable reporter strain had to be constructed, and the $c p c C$ gene had to be identified and comprehensively characterised to result in deletion mutant strains. Accordingly, the impact of $\mathrm{CpcC}$ in virulence was assessed. 
The basal expression level of the Cross-Pathway Control activator CpcA is the main focus of the last chapter. Based on the finding that $c p c C$ deletion strains show no difference in virulence to their wild-type progenitor suggests that the basal expression level of CpcA is necessary but also sufficient to support pulmonary aspergillosis. Conclusively, gene products depending in their expression on basal expression levels of $\mathrm{CpcA}$ should be identified by proteome analyses. 


\subsection{References}

Arndt, K., and Fink, G.R. (1986) GCN4 protein, a positive transcription factor in yeast, binds general control promoters at all 5' TGACTC 3' sequences. Proc Natl Acad Sci U S A 83: 8516-8520.

Arruda, L.K., Mann, B.J., and Chapman, M.D. (1992a) Selective expression of a major allergen and cytotoxin, AspfI, in Aspergillus fumigatus. Implications for the immunopathogenesis of Aspergillus-related diseases. J Immunol 149: 3354-3359.

Arruda, L.K., Platts-Mills, T.A., Longbottom, J.L., el-Dahr, J.M., and Chapman, M.D. (1992b) Aspergillus fumigatus: identification of 16, 18, and $45 \mathrm{kd}$ antigens recognized by human IgG and IgE antibodies and murine monoclonal antibodies. J Allergy Clin Immunol 89: 1166-1176.

Berg, C.M., and Garber, E.D. (1962) A genetic analysis of color mutants of Aspergillus fumigatus. Genetics 47: 1139-1146.

Bishai, W. (2000) Lipid lunch for persistent pathogen. Nature 406: 683-685.

Bodey, G., Bueltmann, B., Duguid, W., Gibbs, D., Hanak, H., Hotchi, M., Mall, G., Martino, P., Meunier, F., Milliken, Naoe, S., Okudaira, M., Scevola, D., and Van't Wout, J. (1992) Fungal infections in cancer patients: an international autopsy survey. Eur $J$ Clin Microbiol Infect Dis 11: 99-109.

Bolard, J. (1986) How do the polyene macrolide antibiotics affect the cellular membrane properties? Biochim Biophys Acta 864: 257-304.

Brajtburg, J., and Bolard, J. (1996) Carrier effects on biological activity of amphotericin B. Clin. Microbiol. Rev. 9: 512-531.

Brakhage, A.A., and Langfelder, K. (2002) Menacing mold: the molecular biology of Aspergillus fumigatus. Annu Rev Microbiol 56: 433-455.

Braus, G.H., Pries, R., Düvel, K., Valerius, O. (2004) Molecular Biology of Fungal Amino Acid Biosynthesis Regulation. In Kück U (ed), The Mycota II, Genetics and Biotechnology, 2nd edn. Springer Press, Berlin Heidelberg New York Tokyo: 239-269.

Brock, M., and Buckel, W. (2004) On the mechanism of action of the antifungal agent propionate. Eur J Biochem 271: 3227-3241.

Bromley, I.M., and Donaldson, K. (1996) Binding of Aspergillus fumigatus spores to lung epithelial cells and basement membrane proteins: relevance to the asthmatic lung. Thorax 51: 1203-1209.

Carsiotis, M., Jones, R.F., and Wesseling, A.C. (1974) Cross-pathway regulation: histidinemediated control of histidine, tryptophan, and arginine biosynthetic enzymes in Neurospora crassa. J Bacteriol 119: 893-898.

Chang, Y.C., Tsai, H.F., Karos, M., and Kwon-Chung, K.J. (2004) THTA, a thermotolerance gene of Aspergillus fumigatus. Fungal Genet Biol 41: 888-896.

Chryssanthou, E. (1997) In vitro susceptibility of respiratory isolates of Aspergillus species to itraconazole and amphotericin B. acquired resistance to itraconazole. Scand J Infect Dis 29: 509-512.

Clements, J.S., Jr., and Peacock, J.E. (1990) Amphotericin B revisited: reassessment of toxicity. Amer J Med 88: 22N-27N.

Cooney, D.G., and Emerson, R., (1964) Thermophilic Fungi. An Account of their Biology, Avtivities and Classification. W.H. Freeman, San Francico, CA.

Stroemnaes I., and Garber E.D. (1962) Heterocariosis and the parasexuel cycle in Aspergillus fumigatus. Genetics 48: 653-662.

D’Enfert, C., Diaquin, M., Delit, A., Wuscher, N., Debeaupuis, J.P., Huerre, M., and Latgé, J.P. (1996) Attenuated virulence of uridine-uracil autotrophs of Aspergillus fumigatus. Infect Immun 64: 4401-4405. 
Davis, R.H., (2000) Neurospora: Contributions of a Model Organism. Oxford, England: Oxford University Press.

Debeaupuis, J.P., Sarfati, J., Chazalet, V., and Latgé, J.P. (1997) Genetic diversity among clinical and environmental isolates of Aspergillus fumigatus. Infect Immun 65: 30803085 .

Denning, D.W. (1998) Invasive aspergillosis. Clin Infect Dis 26: 781-803; quiz 804-785.

Denning, D.W., Venkateswarlu, K., Oakley, K.L., Anderson, M.J., Manning, N.J., Stevens, D.A., Warnock, D.W., and Kelly, S.L. (1997) Itraconazole resistance in Aspergillus fumigatus. Antimicrob. Agents Chemother 41: 1364-1368.

Denning, D.W., Radford, S.A., Oakley, K.L., Hall, L., Johnson, E.M., and, Warnock, D.W. (1997) Correlation between in vitro susceptibility testing to itraconazole and in vivo outcome of Aspergillus fumigatus infection. J Antimicrob Chemother 40: 401-414.

Denning, D.W., Follansbee, S.E., Scolaro, M., Norris, S., Edelstein, H., and Stevens, D.A., (1991) Pulmonary aspergillosis in the acquired immunodeficiency syndrome. $N$ Engl $J$ Med 324: 654-661.

Dixon, D.D., and Walsh, T.J. (1992) Human pathogenesis. Aspergillus, biology and industrial application. Butterworth-Heinemann, Boston, Mass: 249-267.

Drakos, P.E., Nagler, A., Or, R., Naparstek, E., Kapelushnik, J., Engelhard, D., and Rahav, G., Ne'emean, D., and Slavin, S. (1993) Invasive fungal sinusitis in patients undergoing bone marrow transplantation. Bone Marrow Transplant 12: 203-208.

Drysdale, C.M., Jackson, B.M., McVeigh, R., Klebanow, E.R., Bai, Y., Kokubo, T., Swanson, M., Nakatani, Y., Weil, P.A., and Hinnebusch, A.G. (1998) The Gen4p activation domain interacts specifically in vitro with RNA polymerase II holoenzyme, TFIID, and the Adap-Gen5p coactivator complex. Mol Cell Biol 18: 1711-1724.

Dyer, P.S., and Paoletti, M. (2005) Reproduction in Aspergillus fumigatus: sexuality in a supposedly asexual species? Med Mycol 43 Suppl 1: S7-14.

Ebina, K., Yokota, K., and Sakaguchi, O. (1983) Studies on toxin of Aspergillus fumigatus. XVI. Biological properties of Asp-hemolysin as a parasitic factor. Jpn J Med Mycol 24: 247-252.

Eichner, R.D., Al Salami, M., Wood, P.R., and Mullbacher, A. (1986) The effect of gliotoxin upon macrophage function. Int J Immunopharmacol 8: 789-797.

Ellenberger, T.E., Brandl, C.J., Struhl, K., and Harrison, S.C. (1992) The GCN4 basic region leucine zipper binds DNA as a dimer of uninterrupted alpha helices: crystal structure of the protein-DNA complex. Cell 71: 1223-1237.

Ellis, M. (1999) Therapy of Aspergillus fumigatus-related diseases. Contrib Microbiol 2: 105129.

Espinel-Ingroff, A., Fothergill, A., Ghannoum, M., Manavathu, E., Ostrosky-Zeichner, L., Pfaller, M., Rinaldi, M., Schell, W., and Walsh, T. (2005) Quality control and reference guidelines for CLSI broth microdilution susceptibility method (M 38-A document) for amphotericin $\mathrm{B}$, itraconazole, posaconazole, and voriconazole. J Clin Microbiol 43: 5243-5246.

Fukuchi, Y., Kumagai, T., Ebina, K., and Yokota, K. (1996) Apolipoprotein B inhibits the hemolytic activity of asp-hemolysin from Aspergillus fumigatus. Biol Pharm Bull 19: 547-550.

Gil, M.L., Penalver, M.C., Lopez-Ribot, J.L., O'Connor, J.E., and Martinez, J.P. (1996) Binding of extracellular matrix proteins to Aspergillus fumigatus conidia. Infect Immun 64: 5239-5247.

Grant, C.M. (2001) Role of the glutathione/glutaredoxin and thioredoxin systems in yeast growth and response to stress conditions. Mol Microbiol 39: 533-541. 
Grundmann, O., Mösch, H.U., and Braus, G.H. (2001) Repression of GCN4 mRNA translation by nitrogen starvation in Saccharomyces cerevisiae. J Biol Chem 276: 25661-25671.

Haas, H. (2003) Molecular genetics of fungal siderophore biosynthesis and uptake: the role of siderophores in iron uptake and storage. Appl Microbiol Biotechnol 62: 316-330.

Hensel, M., Arst, H.N., Jr., Aufauvre-Brown, A., and Holden, D.W. (1998) The role of the Aspergillus fumigatus areA gene in invasive pulmonary aspergillosis. Mol Gen Genet 258: 553-557.

Hinnebusch, A.G. (1984) Evidence for translational regulation of the activator of general amino acid control in yeast. Proc Natl Acad Sci U S A 81: 6442-6446.

Hinnebusch, A.G. (1997) Translational regulation of yeast GCN4. A window on factors that control initiator-trna binding to the ribosome. J Biol Chem 272: 21661-21664.

Hinnebusch, A.G., and Natarajan, K. (2002) Gcn4p, a master regulator of gene expression, is controlled at multiple levels by diverse signals of starvation and stress. Eukaryot Cell 1: 22-32.

Hoffmann, B., Valerius, O., Andermann, M., and Braus, G.H. (2001) Transcriptional autoregulation and inhibition of mRNA translation of amino acid regulator gene $c p c A$ of filamentous fungus Aspergillus nidulans. Mol Biol Cell 12: 2846-2857.

Hogan, L.H., Klein, B.S., and Levitz, S. M. (1996) Virulence factors of the medically important fungi. Clin. Microbiol. Rev. 9: 469-488.

Hohl, T.M., and Feldmesser, M. (2007) Aspergillus fumigatus: principles of pathogenesis and host defense. Eukaryot Cell 6: 1953-1963.

Hope, W.W., Walsh, T.J., and Denning, D.W. (2005) The invasive and saprophytic syndromes due to Aspergillus spp. Med Mycol 43 Suppl 1: S207-238.

Ibrahim-Granet, O., Dubourdeau, M., Latgé, J.P., Ave, P., Huerre, M., Brakhage, A.A., and Brock, M. (2008) Methylcitrate synthase from Aspergillus fumigatus is essential for manifestation of invasive aspergillosis. Cell Microbiol 10: 134-148.

Jahn, B., Koch, A., Schmidt, A., Wanner, G., Gehringer, H., Bhakdi, S., and Brakhage, A.A. (1997) Isolation and characterization of a pigmentless-conidium mutant of Aspergillus fumigatus with altered conidial surface and reduced virulence. Infect Immun 65: 51105117.

Jahn, B., Boukhallouk, F., Lotz, J., Langfelder, K., Wanner, G., and Brakhage, A.A. (2000) Interaction of human phagocytes with pigmentless Aspergillus conidia. Infect Immun 68: $3736-3739$.

Kamei, K., and Watanabe, A. (2005) Aspergillus mycotoxins and their effect on the host. Med Mycol 43 Suppl 1: S95-99.

Kan, V.L., and Bennett, J.E. (1988) Lectin-like attachment sites on murine pulmonary alveolar macrophages bind Aspergillus fumigatus conidia. J Infect Dis $\mathbf{1 5 8}$ : 407-414.

Kan, V.L., and Bennett, J.E. (1991) beta (1-4) oligoglucosides inhibit the binding of Aspergillus fumigatus conidia to human monocytes. J. Infect. Dis 163: 1154-1156.

Kemper, C.A., Hostetler, J.S., Follansbee, S.E., Ruane, P., Covington, D., Leong, S.S., Deresinski, S.C., and Stevens, D.A. (1993) Ulcerative and plaque-like tracheobronchitis due to infection with Aspergillus in patients with AIDS. Clin Infect Dis 17: 344-352.

Kogan, T.V., Jadoun, J., Mittelman, L., Hirschberg, K., and Osherov, N. (2004) Involvement of secreted Aspergillus fumigatus proteases in disruption of the actin fiber cytoskeleton and loss of focal adhesion sites in infected A549 lung pneumocytes. $J$ Infect Dis 189: 1965-1973. 
Krappmann, S., Bignell, E.M., Reichard, U., Rogers, T., Haynes, K., and Braus, G.H. (2004) The Aspergillus fumigatus transcriptional activator CpcA contributes significantly to the virulence of this fungal pathogen. Mol Microbiol 52: 785-799.

Kurup, V.P., Kumar, A., Kenealy, W. R., and Greenberger, P. A. (1994) Aspergillus ribotoxins react with $\operatorname{IgE}$ and $\operatorname{IgG}$ antibodies of patients with allergic bronchopulmonary aspergillosis. J. Lab. Clin. Med. 123: 749-756.

Kwon-Chung, K.J., and Bennett, J.E. (1992) Medical mycology. Lea \& Febiger, Philadelphia, Pa.

Lamy, B., and Davies, J. (1991) solation and nucleotide sequence of the Aspergillus restrictus gene coding for the ribonucleic toxin restrictocin and its expression in A. nidulans: the leader sequence protects producing strains from suicide. I. Nucleic Acids Res. 19: 1001-1006.

Langfelder, K., Jahn, B., Gehringer, H., Schmidt, A., Wanner, G., and Brakhage, A.A. (1998) Identification of a polyketide synthase gene $(p k s P)$ of Aspergillus fumigatus involved in conidial pigment biosynthesis and virulence. Med Microbiol Immunol 187: 79-89.

Langfelder, K., Philippe, B., Jahn, B., Latgé, J.P., and Brakhage, A.A. (2001) Differential expression of the Aspergillus fumigatus pks $P$ gene detected in vitro and in vivo with green fluorescent protein. Infect Immun 69: 6411-6418.

Latgé, J.P. (1999) Aspergillus fumigatus and aspergillosis. Clin Microbiol Rev 12: 310-350.

Latgé, J.P. (2001) The pathobiology of Aspergillus fumigatus. Trends Microbiol 9: 382-389.

Latgé, J.P., Paris, S., Sarfati, J., Debeaupuis, J.P., Beauvais, A., Jaton-Ogay, K., and Monod M. (1997) Infectivity of Aspergillus fumigatus. In H. Vanden Bossche, D.A. Stevens, and F.C. Odds (ed.), Host-fungusinterplay. National Foundation for Infectious Diseases, Bethesda, Md: 99-110.

Latgé, J.P., Paris, S., Sarfati, J., Debeaupuis, J.P., and Monod, M. (1994) Exoantigens of Aspergillus fumigatus: serodiagnosis and virulence. In K.A. Powell, A. Renwick, and J.F. Peberdy (ed.), The genus Aspergillus: from taxonomy and genetics to industrial application. Plenum Press, London, United Kingdom: 321-340.

Leong, S.A., and Winkelmann, G. (1998) Molecular biology of iron transport in fungi. Met Ions Biol Syst 35: 147-186.

Lorenz, M.C., and Fink, G.R. (2001) The glyoxylate cycle is required for fungal virulence. Nature 412: 83-86.

Lorenz, M.C., and Fink, G.R. (2002) Life and death in a macrophage: role of the glyoxylate cycle in virulence. Eukaryot Cell 1: 657-662.

Lortholary, O., Guillevin, L., and Dupont. B. (1995) Manifestations extrapulmonaires de l'aspergillose invasive. Ann Med Interne 146: 96-101.

Maerker, C., Rohde, M., Brakhage, A.A., and Brock, M. (2005) Methylcitrate synthase from Aspergillus fumigatus. Propionyl-CoA affects polyketide synthesis, growth and morphology of conidia. Febs J 272: 3615-3630.

Maheshwari, R., Bharadwaj, G., and Bhat, M. K. (2000) Thermophilic fungi: their physiology and enzymes. Microbiol Mol Biol Rev 64: 461-488.

Malicev, E., Chowdhury, H.H., Macek, P., and Sepcic, K. (2007) Effect of ostreolysin, an Asp-hemolysin isoform, on human chondrocytes and osteoblasts, and possible role of Asp-hemolysin in pathogenesis. Med Mycol 45: 123-130.

McKinney, J.D., Honer zu Bentrup, K., Munoz-Elias, E.J., Miczak, A., Chen, B., Chan, W.T., Swenson, D., Sacchettini, J.C., Jacobs, W.R., Jr., and Russell, D.G. (2000) Persistence of Mycobacterium tuberculosis in macrophages and mice requires the glyoxylate shunt enzyme isocitrate lyase. Nature 406: 735-738. 
Meletiadis, J., Antachopoulos, C., Stergiopoulou, T., Pournaras, S., Roilides, E., and Walsh, T.J. (2007) Differential fungicidal activities of amphotericin B and voriconazole against Aspergillus species determined by microbroth methodology. Antimicrob Agents Chemother 51: 3329-3337.

Mirande, M. and Waller, J.P. (1988) The yeast lysyl-tRNA synthetase gene. Evidence for general amino acid controlof its expression and domain structure of the encoded protein. J Biol Chem 263: 18443-18451.

Monod, M., Fatih, A., Jaton-Ogay, K., Paris, S., and Latgé, J.P. (1995) The secreted proteases of pathogenic species of Aspergillus and their possiblerole in virulence. Can J Bot 73: 1081-1086.

Morgan, M.A., Wilson, W.R., Neel III, H.B., and Roberts, G.D. (1984) Fungal sinusitis in healthy and immunocompromised individuals. Am J Clin Pathol 82: 597-601.

Mösch, H.U., Scheier, B., Lahti, R., Mantsala, P., and Braus, G. H. (1991) Transcriptional activation of yeast nucleotide biosynthetic gene ADE4 by GCN4. J Biol Chem 266: 20453-20456.

Mullins, J., Harvey, R., and Seaton, A. (1976) Sources and incidence of airborne Aspergillus fumigatus (Fres). Clin Allergy 6: 209-217.

Munoz-Elias, E.J., and McKinney, J.D. (2005) Mycobacterium tuberculosis isocitrate lyases 1 and 2 are jointly required for in vivo growth and virulence. Nat Med 11: 638-644.

Murali, P.S., Kurup, V.P., Guo, J., and Fink, J.N. (1997) Development of bone marrow eosinophilia in mice induced by Aspergillus fumigatus antigens. Clin Immunol Immunopathol 84: 216-220.

Nash, G., Irvine, R., Kerschmann, R.L., and Herndier, B. (1997) Pulmonary aspergillosis in acquired immune deficiency syndrome: autopsy study of an emerging pulmonary complication of human immunodeficiency virus infection. Hum Pathol 28: 12681275 .

Natarajan, K., Meyer, M.R., Jackson, B.M., Slade, D., Roberts, C., Hinnebusch, A.G., and Marton, M.J. (2001) Transcriptional profiling shows that Gcn4p is a master regulator of gene expression during amino acid starvation in yeast. Mol Cell Biol 21: 43474368 .

Nierman, W.C., Pain, A., Anderson, M.J., Wortman, J.R., Kim, H.S., Arroyo, J., Berriman,M., Abe, K., Archer, D.B., Bermejo, C., Bennett, J., Bowyer, P., Chen, D., Collins, M., Coulsen, R., Davies, R., Dyer, P.S., Farman, M., Fedorova, N., Fedorova, N., Feldblyum, T.V., Fischer, R., Fosker, N., Fraser, A., García, J.L., García, M.J., Goble, A., Goldman, G.H., Gomi, K., Griffith-Jones, S., Gwilliam, R., Haas, B.,Haas,H., Harris, D., Horiuchi, H., Huang, J., Humphray, S., Jiménez, J., Keller, N., Khouri, H., Kitamoto, K., Kobayashi, T., Konzack, S., Kulkarni, R., Kumagai, T., Lafton, A., Latgé, J.P., Li, W., Lord, A., Lu, C., Majoros, W.H., May, G.S., Miller, B.L., Mohamoud, Y., Molina, M., Monod, M., Mouyna, I., Mulligan, S., Murphy, L., O’Neil, S., Paulsen, I., Peñalva, M.A., Pertea, M., Price, C., Pritchard, B.L., Quail, M.A., Rabbinowitsch, E., Rawlins, N., Rajandream, M.-A., Reichard, U., Renauld, H., Robson, G.D., Rodriguez de Córdoba, S., Rodríguez-Peña, J.M., Ronning, C.M., Rutter, S., Salzberg, S.L., Sanchez, M., Sánchez-Ferrero, J.C., Saunders, D., Seeger, K., Squares, R., Squares, S., Takeuchi, M., Tekaia, F., Turner, G., Vazquez de Aldana, C.R., Weidman, J., White, O., Woodward, J., Yu, J.-H., Fraser, C., Galagan, J.E., Asai, K., Machida, M., Hall, N., Barrell, B., and Denning, D.W. (2005) Genomic sequence of the pathogenic and allergenic filamentous fungus Aspergillus fumigatus. Nature 438: 1151-1156.

Orciuolo, E., Stanzani, M., Canestraro, M., Galimberti, S., Carulli, G., Lewis, R., Petrini, M., and Komanduri, K.V. (2007) Effects of Aspergillus fumigatus gliotoxin and 
methylprednisolone on human neutrophils: implications for the pathogenesis of invasive aspergillosis. J Leukoc Biol 82: 839-848.

Pagano, L., Ricci, P., Montillo, M., Cenacchi, A., Nosari, A., Tonso, A., Cudillo, L., Chierichini, A., Savignano, C., Buelli, M., Melillo, L., La Barbera, E.O., Sica, S., Hohaus, S., Bonini, A., Bucaneve, G., and Del Favero, A. (1996) Localization of aspergillosis to the central nervous system among patients with acute leukemia: report of 14 cases. Gruppo Italiano Malattie Ematologiche dell'Adulto Infection Program. Clin Infect Dis 23: 628-630.

Panepinto, J.C., Oliver, B.G., Amlung, T.W., Askew, D.S., and Rhodes, J.C. (2002) Expression of the Aspergillus fumigatus rheb homologue, rhbA, is induced by nitrogen starvation. Fungal Genet Biol 36: 207-214.

Panepinto, J.C., Oliver, B.G., Fortwendel, J.R., Smith, D.L., Askew, D.S., and Rhodes, J.C. (2003) Deletion of the Aspergillus fumigatus gene encoding the Ras-related protein $\mathrm{RhbA}$ reduces virulence in a model of Invasive pulmonary aspergillosis. Infect Immun 71: 2819-2826.

Paris, S., Wysong, D., Debeaupuis, J.P., Shibuya, K., Philippe, B., Diamond, R.D., and Latgé, J.P. (2003) Catalases of Aspergillus fumigatus. Infect Immun 71: 3551-3562.

Pathak, A., Pien, F.D. and Carvalho, L. (1998) Amphotericin B use in a community hospital, with special emphasis on side effects. Clin Infect Dis 26: 334338.

Patterson, R., Greenberger, P.A., Radin, R.C., and Roberts, M. (1982) Allergic bronchopulmonary aspergillosis: staging as an aid to management. Ann Intern Med 96: 286-291.

Patterson, R., Greenberger, P.A., Halwig, J.M., Liotta, J.L., and Roberts, M. (1986) Allergic bronchopulmonary aspergillosis. Natural history and classification of early disease by serologic and roentgenographic studies. Arch Intern Med 146: 916-918.

Patterson, T.F. (1998) Editorial response: approaches to therapy for invasive mycoses. The role of amphotericin B. Clin Infect Dis 26: 339-340.

Penalver, M.C., O'Connor, J.E., Martinez, J.P., and Gil, M.L. (1996) Binding of human fibronectin to Aspergillus fumigatus conidia. Infect Immun 64: 1146-1153.

Pfaller, M.A., Messer, S.A., Hollis, R.J., and Jones, R.N. (2002) Antifungal activities of posaconazole, ravuconazole, and voriconazole compared to those of itraconazole and amphotericin B against 239 clinical isolates of Aspergillus spp. and other filamentous fungi: report from SENTRY Antimicrobial Surveillance Program, 2000. Antimicrob Agents Chemother 46: 1032-1037.

Piotrowska, M. (1980) Cross-Pathway regulation of ornithine carbamoyltransferase synthesis in Aspergillus nidulans. J Gen Microbiol 116: 335-339.

Prescott, R.J., Harris, M., and Banerjee, S.S. (1992) Fungal infections of the small and large intestine. J Clin Pathol 45: 806-811.

Ramanan, N., and Wang, Y. (2000) A high-affinity iron permease essential for Candida albicans virulence. Science 288: 1062-1064.

Raper, K.B., and Fennel, D.I. (1965) Aspergillus fumigatus group. In K.B. Raper and D. I. Fennel (ed.), The genus Aspergillus. The William \& Wilkins Co., Baltimore, Md: 238268.

Ratledge, C., and Dover, L.G. (2000) Iron metabolism in pathogenic bacteria. Annu Rev Microbiol 54: 881-941.

Ribaud, P., Chastang, C., Latgé, J.P., Baffroy-Lafitte, L., Parquet, N., Devergie, A., Esperou, H., Selimi, F., Rocha, V., Esperou, H., Selimi, F., Rocha, V., Derouin, F., Socie, G., and Gluckman, E. (1999) Survival and prognostic factors of invasive aspergillosis after allogeneic bone marrow transplantation. Clin Infect Dis 28: 322-330.

Roilides, E., Walsh, T.J., Pizzo, P.A., and Rubin, M. (1991) Granulocyte 
colony-stimulating factor enhances the phagocytic and bactericidal activity of normal and defective human neutrophils. J Infect Dis. 163: 579-583.

Ronning, C.M., Fedorova, N.D., Bowyer, P., Coulson, R., Goldman, G., Kim, H.S., Turner, G., Wortman, J.R., Yu, J., Anderson, M.J., Denning, D.W., and Nierman, W.C. (2005) Genomics of Aspergillus fumigatus. Rev Iberoam Micol 22: 223-228.

Rosenberg, M., Patterson, R., Mintzer, R., Cooper, B.J., Roberts, M., and Harris, K.E. (1977) Clinical and immunologic criteria for the diagnosis of allergic bronchopulmonary aspergillosis. Ann Intern Med 86: 405-414.

Ruijter, G.J., and Visser, J. (1997) Carbon repression in Aspergilli. FEMS Microbiol Lett 151: 103.

Sachs, M.S. (1996) General and cross-pathway controls of amino acid biosynthesis. In: The Mycota: Biochemistry and Molecular Biology, ed. R. Brambl and G.A., Marzluf Heidelberg, Germany: Springer Verlag III: 315-345.

Savage, D.G., Taylor, P., Blackwell, J., Chen, F., Szydlo, R.M., Rule, S.A.J. and A. Spencer, J.F.A., and Goldman, J.M. (1997) Paranasal sinusitis following allogeneic bone marrow transplant. Bone Marrow Transplant 19: 55-59.

Schaffner, A. (1985) Therapeutic concentrations of glucocorticoids suppress the antimicrobial activity of human macrophages without impairing their responsiveness to gamma interferon. J Clin Invest 76: 1755-1764.

Schaffner, A.C.L., Edinburgh, United Kingdom. (1992) Host defense in aspergillosis. In J. E. Bennett, R. J. Hay, and P. K. Peterson (ed.), New strategies in fungal disease. Churchill Livingstone, Edinburgh, United Kingdom: 98-112.

Schöbel, F., Ibrahim-Granet, O., Ave, P., Latgé, J.P., Brakhage, A.A., and Brock, M. (2007) Aspergillus fumigatus does not require fatty acid metabolism via isocitrate lyase for development of invasive aspergillosis. Infect Immun 75: 1237-1244.

Schönheyder, H., Jensen, T., Hoiby, N., and Koch, C. (1988) Clinical and serological survey of pulmonary aspergillosis in patients with cystic fibrosis. Int Arch Allergy Appl Immunol 85: 472-477.

Schrettl, M., Bignell, E., Kragl, C., Joechl, C., Rogers, T., Arst, H.N., Jr., Haynes, K., and Haas, H. (2004) Siderophore biosynthesis but not reductive iron assimilation is essential for Aspergillus fumigatus virulence. J Exp Med 200: 1213-1219.

Serrano-Gomez, D., Dominguez-Soto, A., Ancochea, J., Jimenez-Heffernan, J.A., Leal, J.A., and Corbi, A.L. (2004) Dendritic cell-specific intercellular adhesion molecule 3grabbing nonintegrin mediates binding and internalization of Aspergillus fumigatus conidia by dendritic cells and macrophages. J Immunol 173: 5635-5643.

Shibuya, K., Paris, S., Ando, T., Nakayama, H., Hatori, T., and Latge, J.P. (2006) Catalases of Aspergillus fumigatus and inflammation in aspergillosis. Nippon Ishinkin Gakkai Zasshi 47: 249-255.

Smith, J.M., Tang, C.M., Van Noorden, S., and Holden, D.W. (1994) Virulence of Aspergillus fumigatus double mutants lacking restriction and an alkaline protease in a low-dose model of invasive pulmonary aspergillosis. Infect Immun 62: 5247-5254.

Sugareva, V., Hartl, A., Brock, M., Hubner, K., Rohde, M., Heinekamp, T., and Brakhage, A.A. (2006) Characterisation of the laccase-encoding gene $a b r 2$ of the dihydroxynaphthalene-like melanin gene cluster of Aspergillus fumigatus. Arch Microbiol 186: 345-355.

Tang, C.M., Cohen, J., and Holden, D.W. (1992) An Aspergillus fumigatus alkaline protease mutant constructed by gene disruption is deficient in extracellular elastase activity. Mol Microbiol 6: 1663-1671.

Tang, C.M., Cohen, J., Krausz, T., Van Noorden, S., and Holden, D.W. (1993) The alkaline protease of Aspergillus fumigatus is not a virulence determinant in two murine models of invasive pulmonary aspergillosis. Infect Immun 61: 1650-1656. 
Tekaia, F., and Latgé, J.P. (2005) Aspergillus fumigatus: saprophyte or pathogen? Curr Opin Microbiol 8: 385-392.

Thau, N., Monod, M., Crestani, B., Rolland, C., Tronchin, G., Latgé, J.P., and Paris, S. (1994) rodletless mutants of Aspergillus fumigatus. Infect Immun 62: 4380-4388.

Thireos, G., Penn, M.D., and Greer, H. (1984) 5' untranslated sequences are required for the translational control of a yeast regulatory gene. Proc Natl Acad Sci U S A 81: 50965100 .

Tian, C., Kasuga, T., Sachs, M.S., and Glass, N.L. (2007) Transcriptional Profiling of Cross Pathway Control in Neurospora crassa and Comparative Analysis of the Gen4 and CPC1 Regulons. Eukaryot Cell 6: 1018-1029.

Tournu, H., Tripathi, G., Bertram, G., Macaskill, S., Mavor, A., Walker, L., Odds, F.C., Gow, N.A., and Brown, A.J. (2005) Global role of the protein kinase Gen2 in the human pathogen Candida albicans. Eukaryot Cell 4: 1687-1696.

Tronchin, G., Bouchara, J.P., Larcher, G., Lissitzky, J.C., and Chabasse, D. (1993) Interaction between Aspergillus fumigatus and basement membrane laminin: binding and substrate degradation. Biol Cell 77: 201-208.

Tsai, H.F., Washburn, R.G., Chang, Y.C., and Kwon-Chung, K.J. (1997) Aspergillus fumigatus arpl modulates conidial pigmentation and complement deposition. Mol Microbiol 26: 175-183.

Tsai, H.F., Chang, Y.C., Washburn, R.G., Wheeler, M.H., and Kwon-Chung, K.J. (1998) The developmentally regulated alb1 gene of Aspergillus fumigatus: its role in modulation of conidial morphology and virulence. J Bacteriol 180: 3031-3038.

Van Ho, A., Ward, D.M., and Kaplan, J. (2002) Transition metal transport in yeast. Annu Rev Microbiol 56: 237-261.

Verweij, P.E., Oakley, K.L., Morrissey, J., Morrissey, G., and Denning, D.W. (1998) Efficacy of LY303366 against amphotericin B-susceptible and -resistant Aspergillus fumigatus in a murine model of invasive aspergillosis. Antimicrob Agents Chemother 42: 873878.

Verweij, P.E., and Denning, D.W. (1997) Diagnostic and therapeutic strategies for invasive aspergillosis. Respir Crit Care Med 18: 203-215.

Viollier, A.F., Peterson, D.E., De Jongh, C.A., Newman, K.A., Gray, W.C., Sutherland, J.C., Moody, M.A., and Schimpff, S.C. (1986) Aspergillus sinusitis in cancer patients. Cancer 58: 366-371.

Waring, P., Eichner, R.D., Müllbacher, A., and Sjaarda, A. (1988) Gliotoxin induces apoptosis in macrophages unrelated to its antiphagocytic properties. J Biol Chem 263: 18493-18499.

Washburn, R.G., Kennedy, D.W., Begley, M.G., Henderson, D.K., and, Bennett, J.E. (1988) Chronic fungal sinusitis in apparently normal hosts. Medicine 67: 231-247.

Wingard, J.R., Beals, S.U., Santos, G.W., Merz, W.G., and Saral, R. (1987) Aspergillus infections in bone marrow transplant recipients. Bone Marrow Transplant 2: 175-181.

Yokota, K., Ichinowatari, S., Ebina, K., and Wakabayashi, N. (1985) Studies on toxin of Aspergillus fumigatus. XXI. Site of binding of Asp-hemolysin to erythrocytes and mechanism of inhibition of hemolysis. Jpn J Med. Mycol 26: 70-73.

Zhang, Y.Q., and Keller, N.P. (2004) Blockage of methylcitrate cycle inhibits polyketide production in Aspergillus nidulans. Mol Microbiol 52: 541-550. 


\section{Chapter 2}

\section{CpcA - a Master Regulator of Stress Response Factors}

\subsection{Abstract}

The opportunistic pathogen A. fumigatus requires a variety of metabolic pathways and regulatory circuits to grow and survive in the lungs of infected hosts. One of these is the Cross-Pathway Control (CPC) system, which is homologous to the yeast general control (GC) of amino acid biosynthesis. Based on the observation that the transcriptional activator of the A. fumigatus $\mathrm{CPC}, \mathrm{CpcA}$, is required for full virulence of this fungus, we wanted to find putative CPC-regulated stress genes that are required for infection and survival under stress conditions. In transcriptional profiling studies under amino acid starvation conditions 523 genes were detected that are regulated by $\mathrm{CpcA}$, from which 377 are up-regulated and 146 are repressed. Many of these genes are involved in metabolic pathways like amino acid biosynthesis or sulfur, nitrogen, and carbon metabolism. A large group of permeases and multi-drug transporters as well as transcriptional regulators are also targets of $\mathrm{CpcA}$. Accordingly, these data demonstrate a wide domain regulation executed by the CPC. In addition we detected twelve genes putatively involved in stress response. Two CpcAdependent AAA-ATPases were also present in the data set, which were designated $c d a A$ and $c d a B$. Single and double knock-out mutant strains showed reduced growth on Calcofluor White-containing medium, therefore the corresponding gene products appear to be involved in counteracting cell wall and septa stress. Furthermore, Calcofluor White treatment of A. fumigatus resulted in a weak induction of $c p c A$ transcription but not to an activation of the Cross-Pathway Control system. These studies support the role of the CPC as a global regulatory system evolved in fungi that is active under diverse environmental conditions of stress. 


\subsection{Introduction}

In the last decade the Deuteromycete Aspergillus fumigatus has become one of the most important opportunistic pathogens for immunocompromised hosts such as leukemia patients or recipients of tranplanic organs, which can suffer severely from invasive forms of aspergillosis (Latgé, 1999). The number of infected patients has increased rapidly in the last years with high mortality rates between $30 \%$ to $90 \%$, but until now no effective and reliable therapy against this fungus has been developed (Ellis, 1999). A large number of clinical isolates show resistance to established mycostatical drugs, like the standard triazoles such as itraconazole and voriconazole (Espinel-Ingroff et al., 2005). Other antimycotica, like amphotericine B, that have a higher efficiency and to which less strains show a resistance, may be also toxic for the host itself (Denning et al., 1997; Denning et al., 1997; Meletiadis et $a l$., 2007). Moreover, it is still difficult to diagnose aspergillosis in an early stadium of infection, when a therapy by antifungal pharmaceuticals could lead to a more successful treatment. Therefore, there is still a necessity to find new mycobiotica and therapies that are effective and show no toxicity for the patients. Accordingly, it is important to gain better understanding of A. fumigatus pathogenicity in general and a more detailed picture about factors, pathways, and regulatory systems that are involved in virulence of this saprophytic fungus.

In its natural habitat, which is the soil of decaying organic material like compost, A. fumigatus is able to react on changing growth and stress conditions (Latgé, 1999; Millner et al., 1977; van Heerden et al., 2002). It is also flexible with respect to different temperature ranges, demonstrated by its capacity to grow at levels up to $52-55^{\circ} \mathrm{C}$, whereas other aspergilli like A. flavus, A. niger and A. terreus have not this kind of ability (Chang et al., 2004). Therefore, the body temperature of mammalians does not represent a barrier for this fungus and has no negative influence on the colonisation by the spores of the fungus and the germination within the lung tissue. In order to respond to and to counter naturally occurring environmental stress situations like starvation conditions (Hensel et al., 1998; Krappmann et al., 2004; Panepinto et al., 2002; Panepinto et al., 2003; Ruijter and Visser, 1997), changing temperatures, and escalating $\mathrm{pH}$ values several signalling cascades and distinct cellular pathways are required. The mammalian lung may represent an ecological niche where starvation conditions exist and nutrients are limited; accordingly, the pathways important for survival and adaptation in the natural A. fumigatus habitat may also play a role for germination in the host and therefore as a consequence of invasive aspergillosis. One of these pathways required for adaptation to environmental stress in the natural habitat is the Cross-Pathway Control system (CPC), which 
is homologous to the General Control (GC) of amino acid biosynthesis in S. cerevisisae (Delforge et al., 1975; Hinnebusch, 2005; Krappmann et al., 2004; Schurch et al., 1974; Wolfner et al., 1975). The CPC system consists of two main components, CpcA and CpcC. The encoding $c p c A$ gene is orthologous to yeast GCN4, which codes for a transcriptional regulator that acts on a plethora of genes. $c p c C$ encodes a sensor kinase that is important for translational regulation of $c p c A$ expression in accordance to the yeast mechanism of regulation (Hinnebusch, 2005; Hoffmann et al., 2001). Under sated conditions, two short open reading frames (uORFs) in the upstream region of the $c p c A$ gene repress translation of the $c p c A$ that results in low amounts of $\mathrm{CpcA}$, the basal expression level. Under environmental stress conditions like amino acid starvation, the CPC sensor kinase $\mathrm{CpcC}$ becomes activated to phosphorylate a subunit of the eukaryotic initiation factor of translation eIF2. This results in an increased translation of the transcriptional activator CpcA. Now CpcA can modulate the expression pattern of its target genes enabling the fungus to react to the starvation conditions. In recent studies the yeast general control response under amino acid starvation conditions has been analysed (Hinnebusch, 2005; Natarajan et al., 2001). It was shown that Gcn $4 p$ activates more than 30 genes involved in amino acid biosynthesis in twelve different biosynthetic pathways; around 1000 genes are regulated directly and indirectly by this transcriptional activator under these starvation conditions. Furthermore, the transcriptional response of the Ascomycete Neurospora crassa under amino acid starvation conditions shows de-repressed expression of genes involved in amino acid biosynthesis, which represents the largest group of genes in the according data set (Tian et al., 2007). Genes with functions in carbon metabolism and nitrogen metabolism were also found to be regulated as well as others, which are involved in the oxidative stress response. In total, the CPC-dependent transcriptome of this filamentous fungus shows a regulation of 443 genes. These data sets give a hint on the importance of the fungal CPC network in order to react to different kinds of environmental stress.

In this study the CpcA-dependent transcriptome of A. fumigatus under amino acid starvation conditions was investigated. In earlier works it had been shown that the transcriptional factor $\mathrm{CpcA}$ is involved in pathogenicity of A. fumigatus: a deletion of the encoding gene resulted in attenuated virulence in a murine model of pulmonary aspergillosis (Krappmann et al., 2004). Therefore, the main focus of the data set was set on putative stress factors regulated by $\mathrm{CpcA}$, which maybe support growth of A. fumigatus in the host. Approximately 500 genes were identified to be regulated by $\mathrm{CpcA}$, among them nearly 400 that are influenced in a positive manner. These genes were categorized into different functional groups, such as carbon 
metabolism, amino acid biosynthesis, transporters, which represents the largest group of upregulated genes in the data set, and putative stress genes. Furthermore, the CPC transcriptome was compared with transcriptional profiling data of $A$. fumigatus propagated in the presence of the antifungal drug voriconazole (da Silva Ferreira et al., 2006). 


\subsection{Materials and Methods}

\subsubsection{Strains and media}

For general cloning procedures the Escherichia coli strain DH5a [F-,f80dD(lacZ)M15-1, $\mathrm{D}($ lacZYA-argF)U169, recAl, endA1,hsdR17 (rK-, mK+), supE44, 1-, thil, gyrA96, relA1] (Woodcock et al., 1989) was used. E. coli cultures were grown in LB or LBLS (1\%bactotryptone, $0.5 \%$ yeast extract, $1 \%$ or $0.5 \% \mathrm{NaCl}, \mathrm{pH} 7.5$ ) medium at $37^{\circ} \mathrm{C}$. Fungal strains used in this study are listed in table 2.1 Growth of $A$. fumigatus strains was carried out at $37^{\circ} \mathrm{C}$ on minimal medium prepared and supplemented according to Käfer (1977). Supplemented antibiotics and drugs were ampicillin $(100 \mu \mathrm{g} / \mathrm{ml})$, kanamycin $(50 \mu \mathrm{g} / \mathrm{ml})$, hygromycin B (200 $\mu \mathrm{g} / \mathrm{ml})$, phleomycin $(40 \mu \mathrm{g} / \mathrm{ml})$, pyrithiamine $(100 \mu \mathrm{g} / \mathrm{ml})$.

\subsubsection{A. fumigatus transformation and plasmid construction}

Aspergillus DNA was extracted and isolated according to the protocol of Kolar et al. (1988). For genomic analysing Southern hybridisation method was used (Southern, 1975), the probe labelling was done with the DNA labelling kit of GE Healthcare (Buckinghamshire, UK) was used according to the manufacturer's protocol. For DNA amplification Phusion polymerase of FINNZYMES was used as described previously (Saiki et al., 1985).

E. coli transformation was performed according to the protocol of Higa and Mandel (1970). Aspergillus strains were transformed by using the polyethylene glycol-mediated fusion of protoplasts (Punt and van den Hondel, 1992).

For constructing the $c d a A$ knock out cassette the 5' UTR of the gene was amplified by using the primer pair CS49/CS50 (3.4 kb). The 3' region was amplified by using the primers CS51/CS52 $(2.7 \mathrm{~kb})$. The hygromycin resistance cassette was received by digesting pME3002 with $S f i \mathrm{I}(3.5 \mathrm{~kb})$. All three received fragments were ligated via overlapping SfiI sites using a T4 ligase receiving a 5'-marker-3' fragment and transformed in the $\mathrm{pCR}^{\circledR}$-BluntII-TOPO ${ }^{\circledR}$ vector (INVITROGEN GMBH, Karlsruhe, D) according to the manufacturer's protocol. For construction of the $c d a B$ deletion cassette the flanking regions of $c d a B$ were amplified by using the primers CS45/CS46 for the 5' UTR (2 kb) and CS47/CS48 for the 3' region (2.1 $\mathrm{kb})$. A phleomycin resistance cassette was received by digesting pME2891 with SfiI (2.9 kb). Via ligation of the three fragments the construct 5'-marker-3' was received and finally cloned in pBlueskript II KS via EcoRV (pCS8). Used primers and plasmids are listed in table 2.2 and 2.3 To delete $c d a A$ pCS5 $\left(\right.$ pCR $^{\circledR}$-BluntII-TOPO ${ }^{\circledR}$ vector containing the $c d a A$ deletion cassette) was digested with FspI and StuI to receive an $8.9 \mathrm{~kb}$ fragment 5'-marker-3', which was transformed via homologous integration in the clinical isolate D141 (AfS00). pCS8 
(pBlueskript II KS containing the $c d a B$ deletion cassette) was digested with HpaI and NheI receiving a $6.9 \mathrm{~kb}$ fragment, which was transformed via homologous recombination in the wild-type strain D141(AfS00). For construction of the double knock out strain the cdaB deletion construct was integrated via homologous recombination in the $c d a A$ deletion strain. For all polymerase chain reactions genomic DNA was used as template. For marker recycling the protocol was used according to Krappmann et al. (2005) with the modification to select transformants on minimal medium containing $25 \mu \mathrm{M}$ FUDR.

\subsubsection{Growth tests}

2000 spores in $2 \mu \mathrm{l}$ of each strain were plated on minimal medium (MM) and incubated for two to three days at $37^{\circ} \mathrm{C}$. For amino acid starvation condition $2 \mathrm{mM} \mathrm{L}$-methione sulfoximine (MSX) were used. $1 \mathrm{mg} / \mathrm{ml}$ Calcofluor White (SIGMA) containing medium was used to induce stress conditions to cell wall and septa building.

\subsubsection{Transcriptome analysis and Northern hybridisation}

$2 \times 10^{6}$ spores per millilitre of the wild-type strain D141 and the cpcA deletion strain AfS01 were inoculated in liquid MM containing ammonium as nitrogen source. After $17 \mathrm{~h}$ incubation at $37^{\circ} \mathrm{C}$ the cultures were shifted for additional $4 \mathrm{~h}$ to fresh $\mathrm{MM}$ in the presence or absence of $10 \mathrm{mM}$ 3-amino-1,2,4-triazol (3AT) to evoke amino acid starvation conditions. Mycelia were harvested by filtration with miracloth filter (CALBIOCHEM) and washed thoroughly with sterile water. Total RNA was isolated from liquid cultures by using Trizol (INVITROGEN). $20 \mu \mathrm{g}$ RNA from each sample were fractionated in formaldehyde, 1,4\% agarose gel, stained with ethidium bromide and the visualised with UV-light. The presence of clear ribosomal bands was used as a criterion for good quality. Each RNA sample was digested with DNAse and purified with RNeasy columns from Qiagen and eluted in $70 \mu \mathrm{l}$ RNAse free water. For hybridisation microarray chips for A. fumigatus Af293 from TIGR were used. Hybridisation was accomplished according to the protocol of da Silva Ferreira et al. (2006). For verification of the transcriptome data and induction under Calcofluor White conditions Northern experiments according to the protocols cited by Brown and Mackey (1997) were done. Random primed labelling was performed with the STRATAGENE Prime-It II kit in the presence of $[\alpha-32 \mathrm{P}]-\mathrm{dATP}$. (Feinberg and Vogelstein, 1983). Autoradiographies were produced by exposing the washed membranes to KODAK X-OMAT films. 
Table 2.1. List of used strains in this study

\begin{tabular}{|l|l|l|}
\hline Strain & Description & Reference \\
\hline D141 & wildtype, clinical isolate $^{\prime}$ & Staib et al., 1980 \\
\hline AfS01 & $\Delta c p c A ;$ phleo $^{R}$ & Krappmann et al., 2004 \\
\hline AfC07 & $\Delta c d a A ;$ hygr $^{R}$ & this study \\
\hline AfC08 & $\Delta c p c B ;$ phleo $^{R}$ & this study \\
\hline AfC09 & $\Delta c d a A ;$ hygr $^{R} ; \Delta c p c B ;$ phleo $^{R}$ & this study \\
\hline AfC10 & $c d a A::$ loxP & this study \\
\hline AfC11 & $c d a B:: \operatorname{loxP}$ & this study \\
\hline AfC12 & $\Delta c d a A ;$ hygr $^{R} ; c d a B::$ loxP & this study \\
\hline
\end{tabular}

Table 2.2. List of used plasmids in this study

\begin{tabular}{|c|c|c|}
\hline Plasmid & Description & Reference \\
\hline pBluescript $^{\circledR} \mathrm{II} \mathrm{KS}^{+}$ & cloning vector $\left(a m p^{R} ; \mathrm{MCS}\right)$ & Stratagene (La Jolla, CA, USA) \\
\hline pCR $^{\circledR}$-BluntII-TOPO ${ }^{\circledR}$ & cloning vector $\left(\mathrm{kan}^{R}\right)$ & Invitrogen Gmbh (Karlsruhe, D) \\
\hline pCS5 & $c d a A$ deletion cassette $(c d a A: \because l o x P-h p h / t k)$ & this study \\
\hline pCS8 & $c d a B$ deletion cassette $(c d a B::$ loxP-ble/tk) & this study \\
\hline pME2891 & loxP-pgpdA::ble/HSV1tk::trpCt-loxP & Krappmann et al., 2005 \\
\hline pME2892 & Cre expression module in pPTRII & Krappmann et al., 2005 \\
\hline pME3002 & loxP-pgpdA::hph/HSV1tk::trpCt-loxP & Krappmann et al., 2005 \\
\hline
\end{tabular}

Table 2.3. Primers used in this study

\begin{tabular}{|c|c|c|}
\hline Name & Size & Sequence \\
\hline CS13 & 19-mer & 5'-TGCATCCTGCGCTTCGATG-3' \\
\hline CS14 & 19-mer & 5'-CAGAATACGCCACGCGAAG-3' \\
\hline CS17 & 20-mer & 5'-TTCATCCTCTTCCCCACTGG-3' \\
\hline CS18 & 23-mer & 5'-ACTACGGAAGAAGCGGTCCA-3' \\
\hline CS21 & 20-mer & 5'-ATACGACCAAATCGATGTCC-3' \\
\hline CS22 & 20-mer & 5'-AAGTTCAGACTGATCACGTC-3' \\
\hline CS25 & 20-mer & 5'-AAG GTTCCTTTCGTCTGTGG-3' \\
\hline CS26 & 20-mer & 5'-GGTCCTTGTAGTGAGTCACG-3' \\
\hline CS45 & 20-mer & 5'-AGCTGCTTTGTCACTTCCAG-3' \\
\hline CS46 & 35-mer & 5'-TAGGCCTGAGTGGCCGAAGGAGGATTCCTTGGTGG-3' \\
\hline CS47 & 38-mer & 5'-TAGGCCATCTAGGCCTACGTCACACGCCAATCCAAATC-3' \\
\hline CS48 & 19-mer & 5'-CGGAAACGGAGCGAATTAG-3' \\
\hline CS49 & 18-mer & 5'-CGTGCCTGAGGATCCTCC-3' \\
\hline CS50 & 35-mer & 5'-TAGGCCTGAGTGGCCAGGGGAAACCTAAGGTAGAG-3' \\
\hline CS51 & 34-mer & 5'-TAGGCCATCTAGGCCCGAGGAAATCATTCAAAGAC-3' \\
\hline CS52 & 20-mer & 5'-GGAAGCAAGACAATCTCTGA-3' \\
\hline
\end{tabular}


Chapter 2

\begin{tabular}{|l|l|l|}
\hline CS76 & 19-mer & 5'-GAGCTACGGGATACGATTG-3' \\
\hline
\end{tabular}

Table 2.3 continued.

\begin{tabular}{|l|l|l|}
\hline Name & Size & Sequence \\
\hline CS77 & 19 -mer & 5'-ACAAGTCCGTTGAGTTGCG-3' \\
\hline CS31 & 20 -mer & 5'-ATCCATCGTCGCATCAACCC-3' \\
\hline CS75 & 18 -mer & 5'-TGGCGGCCGTTCAGATTG-3' \\
\hline VG20 & 18 -mer & 5'-CTGTTCACGCTGCTGAGC-3' \\
\hline Sv27 & 20 -mer & 5'-TCAATTCACTCAAAAACAGC-3' \\
\hline Sv48 & 23 -mer & 5'-TCTGGAGGATACAATCACTTTCC-3' \\
\hline Sv49 & 23 -mer & 5'-AATCCCATTGAGTGCCTTTCAGC-3' \\
\hline Sv64 & 23 -mer & 5'-TAATCTATTCAAAAGATCTGAGG-3' \\
\hline Sv65 & 21 -mer & 5'-TCTACACACAGATCTAGTTGG-3' \\
\hline
\end{tabular}




\subsection{Results}

In the yeast S. cerevisiae it was shown that the general control of amino acid biosynthesis with its transcriptional regulator Gcn4p regulates a diverse set of genes and cellular pathways with a certain bias to those involved in amino acid biosynthesis (Delforge et al., 1975; Hinnebusch, 2005; Schurch et al., 1974; Wolfner et al., 1975). Earlier works on transcriptional profiling in dependency of Gen $4 p$ revealed that more than 600 genes are regulated directly or indirectly by this transcriptional activator (Natarajan et al., 2001). The homologous systems to the general control can also be identified in other fungi like $N$. crassa and A. nidulans, where it is called Cross-Pathway Control (CPC) (Carsiotis et al., 1974; Davis, 2000; Piotrowska, 1980; Sachs, 1996). In A. fumigatus, this system was investigated under the aspect for its role in virulence; thus, previous works showed that the transcriptional regulator $\mathrm{CpcA}$, the orthologue to $\mathrm{Gcn} 4 \mathrm{p}$, is required for full pathogenicity of A. fumigatus (Krappmann et al., 2004). Therefore, it was of interest to get an overview of the CpcAdependent transcriptome with the focus set on stress-related genes. In this study a comparison between a wild-type isolate of A. fumigatus and its $\triangle c p c A$ derivative under conditions of histidine starvation was done.

\subsubsection{Transcriptional profiling of histidine-starved A. fumigatus reveals the CpcA- dependent transcriptome}

To analyse the CpcA-dependent transcriptome the wild-type clinical isolate D141 and the $\triangle c p c A$ mutant AfS01 were propagated in liquid minimal medium and shifted to conditions of histidine starvation for four hours induced by the antimetabolite 3AT. Competitive hybridisations on whole genome microarrays were carried out (see Material and Methods) to compare the transcriptional profiles of each strain in the absence or presence of amino acid starvation. Analysis of both transcriptome data sets revealed 1563 genes to be regulated to a certain extent (see supplemental table 1). For further analyses, only genes above a threshold value of 1.5 fold regulation were considered. Accordingly, 1048 entries from the initial gene list showed at least an expression level of 1.5 fold or higher (suppl. table 2). In the wild-type 842 genes were found, from which 494 were induced and 348 were repressed in the presence of 3AT; in the $\triangle c p c A$ strain 493 genes were regulated, from which 195 were up-regulated and 298 down-regulated. Only 290 genes showed in both strains the same regulation. 555 genes are higher expressed in the wild-type in comparison to the $\Delta c p c A$ mutant and 206 are only present in the $\triangle c p c A$ mutant data set (suppl. tables 3 and 4). 


\subsubsection{Estimation of the CpcA-dependent transcriptome}

In order to identify direct and indirect targets of $\mathrm{CpcA}$, all genes with a regulation level of 1.5 fold or higher (1048) were screened for being regulated in the wild-type under starvation condition but not in the $\triangle c p c A$ strain. 523 gene loci could be assigned to this analysis, resulting in the suggestion that this group contains genes that are regulated by the CPC effector CpcA (suppl. table 5). For validation purposes, the transcriptional steady-state level of some genes induced under starvation was checked in Northern hybridisations. As internal standard a probe binding to the CpcA-independent aroC transcript was used. All of the analysed genes were highly expressed in the wild-type under starvation but not under normal conditions. Altered expression levels of these genes in the $\triangle c p c A$ strain were neither seen under starvation nor under non-starvation conditions (fig. 2.1).

The set of 523 genes was classified in functional categories. The majority (377 genes) showed an up regulation in the presence of amino acid starvation (table 2.4 and suppl. table 6), whereas only 146 were repressed under these conditions (suppl. table 7). 49 of the 377 upregulated genes are involved in amino acid biosynthesis to form the largest functional group of the data set. $48 \mathrm{CpcA}$-regulated genes encoding putative transporters or permeases could be identified. Also a high number (25) of transcriptional and translational factors were positively regulated by $\mathrm{CpcA}$, corroborating the assumption that $\mathrm{CpcA}$ is a master regulator of the fungus. Additionally, genes involved in carbon, nitrogen, and sulfur metabolism could be assigned; in total, 19 genes encoding proteins for these metabolic pathways were detected in the data set. Moreover, factors involved in vitamin and cofactor biosynthesis, fatty acid and purin/pyrimidin metabolism, and secondary metabolism were de-repressed in a CpcAdependent manner under amino acid starvation conditions as well as genes encoding enzymes related to protein degradation and modification. Furthermore, many genes required for other functionalities were identified, and additional hypothetical genes or genes with unknown function could be found in the data set. 


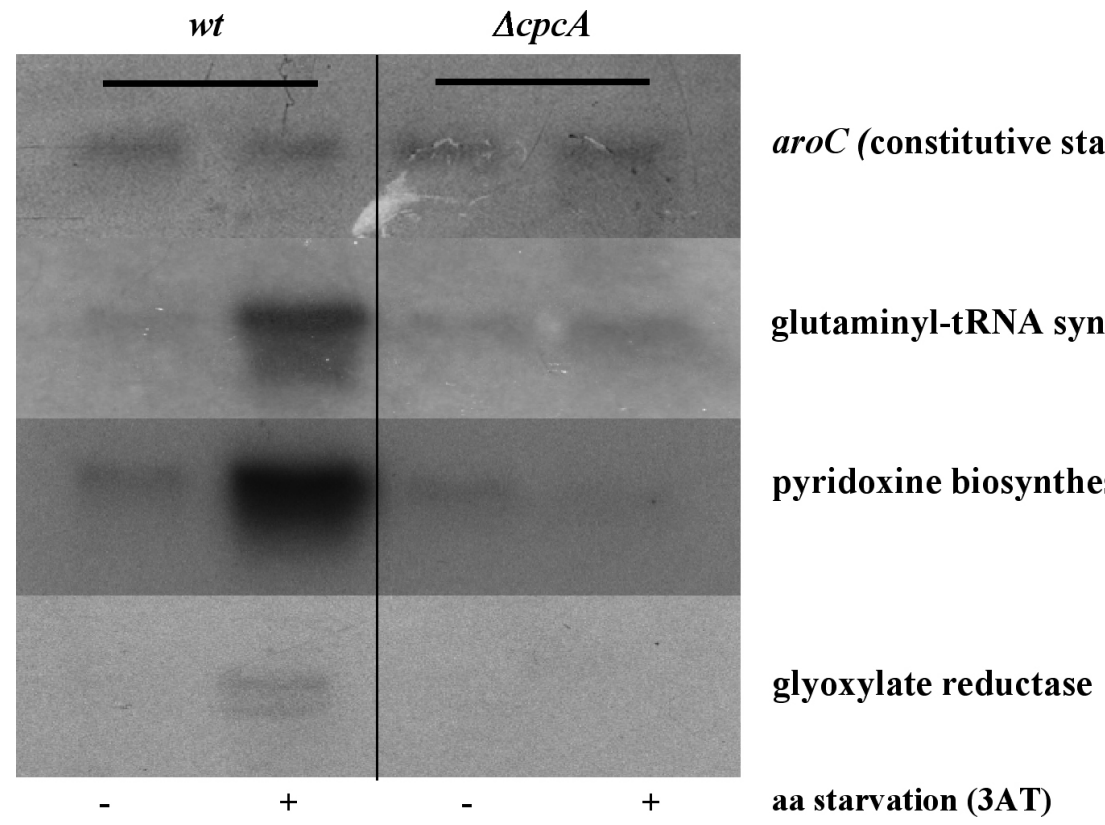

Figure 2.1. Validation of the transcriptome profiling data set

Northern hybridisation patterns of several genes that showed an up-regulation in the transcriptome profile using RNA samples from wild-type (wt) and the $\Delta c p c A$ deletion strain in the presence or absence of amino acid starvation conditions. In accordance with the microarray hybridisation data, the selected genes display increased transcription under starvation in the wild-type but not in the deletant.

In further analyses, the focus was set on putative CpcA targets that are likely to be involved in any kind of stress response that would support survival of $A$. fumigatus under conditions of environmental stress. In the profiling data, twelve targets could be identified fulfilling this condition (table 2.5).

Table 2.4. Numbers of CpcA-induced genes assigned to functional categories.

\begin{tabular}{|l|c|c|}
\hline Category & Number & \% of all 377 induced genes \\
\hline hypothetical proteins & 76 & $20.2 \%$ \\
\hline aa biosynthesis & 49 & $13.0 \%$ \\
\hline transporters/permeases & 48 & $10.3 \%$ \\
\hline other genes & 39 & $6.6 \%$ \\
\hline transcription-/translation factors & 25 & $6.6 \%$ \\
\hline genes with unknown function & 25 & $3.7 \%$ \\
\hline acetyl-/methyltransferases & 14 & $3.4 \%$ \\
\hline carbon meatbolism & 13 & $3.2 \%$ \\
\hline stress response factors & 12 & $2.7 \%$ \\
\hline purine/pyrimidine-modification/DNA/RNA & 10 & $2.4 \%$ \\
\hline protein degradation & 9 & $2.1 \%$ \\
\hline oxidoreductases involved in metabolism & 8 & $2.1 \%$ \\
\hline tRNA modification/synthesis & 8 & \\
\hline
\end{tabular}


Table 2.4 continued.

\begin{tabular}{|l|c|c|}
\hline Category & Number & \% of all 377 induced genes \\
\hline replication and repair systems DNA/RNA & 7 & $1.9 \%$ \\
\hline coenzyme sythesis & 7 & $1.9 \%$ \\
\hline nitrogen and sulfur metabolism & 6 & $1.1 \%$ \\
\hline secondary metabolism & 4 & $1.1 \%$ \\
\hline heavy metal binding $(\mathrm{Cu}, \mathrm{Fe}, \mathrm{Zn})$ & 3 & $8.0 \%$ \\
\hline cell growth & 3 & $8.0 \%$ \\
\hline ribosomal proteins & 3 & $8.0 \%$ \\
\hline respiration & 3 & $8.0 \%$ \\
\hline fatty acid metabolism & 3 & $8.0 \%$ \\
\hline vitamin biosythesis & 1 & $0.3 \%$ \\
\hline cell wall & & \\
\hline
\end{tabular}

Table 2.5. CpcA-dependent A. fumigatus genes putatively involved in stress response.

\begin{tabular}{|l|l|}
\hline Locus tag & Gene \\
\hline Afu7g05750 & Afu7g05750 AAA family ATPase, putative \\
\hline Afu3g10770 & Afu3g10770 RTA1 domain protein, putative \\
\hline Afu5g07960 & Afu5g07960 C2H2 finger and ankyrin domain protein, putative \\
\hline Afu7g06570 & Afu7g06570 zinc/cadmium resistance protein \\
\hline Afu1g16560 & Afu1g16560 MIF domain protein \\
\hline Afu1g05040 & Afu1g05040 protein mitochondrial targeting protein (Mas1), putative \\
\hline Afu7g06680 & Afu7g06680 AAA family ATPase, putative \\
\hline Afu2g00590 & Afu2g00590 glutathione-S-transferase, putative \\
\hline Afu6g08460 & Afu6g08460 cytochrome P450 alkane hydroxylase, putative \\
\hline Afu3g10220 & Afu3g10220 acid phosphatase, putative \\
\hline Afu5g13000 & Afu5g13000 CRAL/TRIO domain protein \\
\hline Afu2g11180 & Afu2g11180 developmental regulator FlbA \\
\hline
\end{tabular}

In the data set CpcA repressed the expression of 146 genes in the presence of 3AT (table 2.6); 17 of these genes encode transporters or are involved in transport mechanisms. In addition, eleven genes related to carbon metabolism, which was nearly the same number of genes that were up-regulated under starvation were found. In contrast to that, no gene involved in amino acid biosynthesis was found to be repressed and only four transcriptional factors showed repression in a $\mathrm{CpcA}$-dependent manner. Also the identification of annotated genes in the repression data set having a function in nitrogen or sulfur metabolism failed. The comparison of genes involved in protein metabolism and degradation revealed to no obvious difference between the wild-type and the deletion strain, since in both data sets targets of these functions 
were found. Interestingly, genes classified to be involved in stress-response were also downregulated in dependency of $\mathrm{CpcA}$. However, these were mainly proteins assigned to chaperone function in order to react on stress induced by heat, whereas in the other data set a strong diversity predominated.

Table 2.6. Numbers of $\mathrm{CpcA}$-dependent repressed genes within different functional categories.

\begin{tabular}{|l|c|c|}
\hline Category & Number & \% of all 146 repressed genes \\
\hline hypothetical proteins & 45 & $30.8 \%$ \\
\hline transporters/permeases & 17 & $11.6 \%$ \\
\hline other genes & 14 & $9.6 \%$ \\
\hline genes with unknown function & 13 & $8.9 \%$ \\
\hline stress/heat shock & 12 & $8.2 \%$ \\
\hline carbon meatbolism & 11 & $7.5 \%$ \\
\hline protein degradation & 6 & $4.1 \%$ \\
\hline transcriptionsfactors & 4 & $2.7 \%$ \\
\hline DNA mod./RNA mod./ & 4 & $2.7 \%$ \\
\hline ribosomal proteins & 3 & $2.1 \%$ \\
\hline oxidoreductases involved in metabolism & 3 & $2.1 \%$ \\
\hline cell wall & 3 & $2.1 \%$ \\
\hline fatty acid metabolism and ureat cycle & 3 & $2.1 \%$ \\
\hline Acetyl-/Methyltransferasen & 3 & $2.1 \%$ \\
\hline respiration & 2 & $1.4 \%$ \\
\hline heavy metal binding (Cu, Fe, Zn) & 2 & $1.4 \%$ \\
\hline nitrogen and sulfur metabolism & 1 & $0.7 \%$ \\
\hline pH regulation & 1 & $0.7 \%$ \\
\hline
\end{tabular}

\subsubsection{The CpcA-directed transcriptome of A.fumigatus in comparison to the} transcriptional profile elicited by voriconazole treatment

Amino acid starvation represents a stress condition to A. fumigatus that triggers a cellular response by de-repression of $\mathrm{CpcA}$ expression to result in regulation of various kinds of genes. This wide domain response enables the fungus to react on the encountered stress signal. In order to assess some overlap of the CpcA-directed transcriptome and the one elicited by the antifungal drug voriconazole, the data were compared to the transcriptional profile, in which A. fumigatus was incubated for $4 \mathrm{~h}$ in the presence of this antifungal drug (da Silva Ferreira et al., 2006). Voriconazole belongs to the azole family of antifungal drugs, which target the fungal ergosterol biosynthesis (Diaz-Guerra et al., 2003). Unknown and 
hypothetical genes from the previous data set were not taken in consideration for the comparison; among the remaining genes that were induced in the CpcA-directed transcriptome, 46 showed also an up-regulation of 1.5 fold or higher in the presence of voriconazole whereas 37 were repressed. In the presence of 3AT, the transcriptional level of 48 amino acid biosynthesis proteins was increased in the wild-type, with only three of them being also induced by voriconazole treatment. However, the remaining 45 amino acid biosynthesis genes were repressed or displayed no induction of 1.5 fold or higher by voriconazole. The second largest group in the profile of induced genes were putative transporters and permeases. Seven of them were down-regulated, and only five displayed an induction of expression during incubation with voriconazole. Interestingly, five of the twelve putative CpcA-regulated stress genes were induced in the voriconazole profile as well, while seven showed a diminished expression level below the threshold of 1.5 fold regulation or they were not detectable in the voriconazole transcriptome profile (table 2.7). A further interesting fact was the pronounced up-regulation of the $c p c A$ gene itself during voriconazole treatment, leading to the suggestion that this drug acts on $c p c A$ expression on the level of transcription. However, expression of the $\arg B$-encoded ornithine carbamoylase activity was not detectable in the presence of the antifungal drug, which indicates that voriconazole increases the transcription of the CPC regulator but does not trigger the CPC response itself.

Table 2.7. Stress genes induced under amino acid starvation and voriconazole-induced stress

\begin{tabular}{|l|l|c|c|}
\hline Locus tag & Gene & 3AT $^{*}$ & Voriconazole $^{*}$ \\
\hline Afu7g05750 & AAA family ATPase, putative & 5.40 & 2.69 \\
\hline Afu3g10770 & RTA1 domain protein, putative & 3.98 & 3.84 \\
\hline Afu5g07960 & C2H2 finger and ankyrin domain protein, putative & 3.49 & 1.76 \\
\hline Afu7g06570 & zinc/cadmium resistance protein & 5.00 & 4.58 \\
\hline Afu7g06680 & AAA family ATPase, putative & 3.90 & 49.03 \\
\hline
\end{tabular}

*induction factor of the gene ater treatment with $3 \mathrm{AT} /$ voriconazole

\subsubsection{CpcA-dependent stress genes}

A dozen genes were identified that are likely to be involved in the fungal stress response (see table 2.5) among them a putative glutathione-S-transferase. In A. fumigatus three functional glutathione transferases were characterised by Burns et al. (2005) to be involved in detoxification of reactive oxidative species (ROS) like $\mathrm{H}_{2} \mathrm{O}_{2}$ and of xenobiotics. This detoxification function is similar to the function of cytochrome P450 alkane hydroxylase, another putative stress related gene found in our transcriptional profile. Additional functional stress response genes were up-regulated in our data set, for example a protein containing an 
RTA1 domain. It is known from yeast that specific proteins containing such an RTA1 domain enable the organism to react on triazole-containing fungal drugs like itraconazole, resulting in increased resistance. From the pathogenic yeast C. glabrata it is known that RTAl is a target of Pdr1, a transcriptional regulator for pleiotropic drug resistance (Vermitsky et al., 2006). In addition to these stress response proteins, a zinc/cadmium resistance protein encoded by the gene with the locus tag Afu7g06570 was identified. Previous studies had indicated that although these kinds of gene products are not unconditional essential for the organism they are often required for normal growth under stress conditions. Deletion of these genes may lead to accumulation of zinc, which results in a higher sensitivity towards other growthinhibiting drugs. This was shown, for instance, for a zinc transporter in S. cerevisiae encoded by the YKE4 gene, whose deletion results in zinc accumulation accompanied by a higher sensitivity to Calcofluor White in medium that also contains increased concentrations of zinc (Kumanovics et al., 2006). A further interesting gene found in the data encodes a regulator involved in development $(f l b A)$. This gene, which belongs to the family of regulators of Gprotein signalling (RGS) was characterised in A. nidulans as a negative regulator for vegetative growth and to be involved in signalling in order to react on changing environmental conditions (Lee and Adams, 1994; Yu et al., 1996). The other RGSs rgsA, $\operatorname{rgs} B$ and $\operatorname{rgs} C$, which are also known from A. nidulans (Han et al., 2004) were however not detected in the data set.

Among the twelve different stress genes, two genes encoding ATPases belonging to the AAA-ATPases family are present. Members of the AAA-ATPase family are associated to various cellular activities like chaperone activity, proteolysis and movement of microtubule motors in eukaryotes. Most of these proteins undergo a conformation change and/or bind ATP for hydrolysation, resulting in physical works; however, the exact mechanism of conformational changing is still not completely clarified. In general, proteins of the AAAATPases family consists of six different domains, and they have the ability to form oligomers, in general hexamers. Most eukaryotic genomes encode 50 to 80 members of this protein family (Hanson and Whiteheart, 2005). Accordingly, BLAST analysis using the typical conserved AAA-motif, as input query was carried out against the A. fumigatus genome to retrieve eleven annotated gene loci encoding putative members of this family (table 2.8). Interestingly, two of these ATPases were induced in the transcriptional profiling studies, accordingly they were designated $c d a A$ (Afu7g06680) and $c d a B$ (Afu7g05750) for $C \mathrm{pcA}$ dependent AAA+ ATPases. For further investigations and characterisation the up-regulation of these both genes upon 3AT treatment in a CpcA-dependent manner had to be validated. 
Table 2.8. AAA ATPases of A. fumigatus

\begin{tabular}{|l|l|l|l|}
\hline Afu1g02060 & Afu2g12920 & Afu4g11180 & Afu7g06680 \\
\hline Afu1g02410 & Afu4g04800 & Afu6g12560 & Afu8g04270 \\
\hline Afu1g09210 & Afu4g10730 & Afu7g05750 & \\
\cline { 1 - 3 } & &
\end{tabular}

The genes induced under amino acid starvation conditions and by voriconazole-treatment are written in bold.

\subsection{5 $c d a A$ and $c d a B$ appear to be regulated by the transcriptional activator CpcA}

To check the induction of both AAA-ATPases in dependency of CpcA under starvation conditions, Northern hybridisation experiments were carried out. Therefore, the wild-type isolated D141 and in parallel the $c p c A$ deletion strain AfS01 were cultivated in liquid culture in the absence or presence of 3AT. As internal standard, transcript levels of the CpcAindependent gene aroC were used (fig. 2.2A). The wild-type displayed increased signal intensities for both AAA-ATPases in comparison to the sample without 3AT, and no signal could be detected for the $\triangle c p c A$ strain in the presence of this antimetabolite. To exclude that this was a phenomenon depending specifically on 3AT, further controls were performed, but in contrast to the previous ones MSX (L-Methione sulfoximine) was used as inducer of the CPC. This drug inhibits the glutamine synthetase to result in glutamine starvation. With respect to $\mathrm{CpcA}$-dependent regulation of transcription, no difference to the 3AT-treated samples were observed for $c d a A$. The $c d a B$ gene, however, showed under this condition not only a prominent signal in the wild-type but also in the mutant (fig. 2.2B). These data indicate that expression of both genes is affected by amino acid starvation conditions in a positive manner; however, only for the $c d a A$-encoded AAA+ ATPase a CpcA-dependency gene regulation could be validated.

For both gene products the AAA motif was found at the C-terminus, and each encoding gene consists of two exons separated by one intron. In addition the promoter regions of these genes within 1000 basepairs (bp) were analysed for typical cpcA binding sites (5'-TGACTCA-3'; 5'-TGACTgA-3'; 5'-TGACTgA-3'; 5'-TGAgTgA-3'; Tian et al., 2007) to support whether they are putative targets of $\mathrm{CpcA}$. For both genes, one conserved binding site could be detected. However, only in the $c d a A$ promoter region a recognition site of the canonical 5'TGACTCA-3' sequence was identified, whereas in the $c d a B$ upstream region a site of the less typical CpcA binding region 5'-TGAgTgA-3' was found (fig. 2.3). This result hints towards a direct regulation of the $c d a A$ gene by the transcriptional activator $\mathrm{CpcA}$ and a less stringent one for the $c d a B$ gene. Furthermore, we were interested in the cellular function of both ATPases in A. fumigatus; thus, both genes were deleted in additional experiments. 

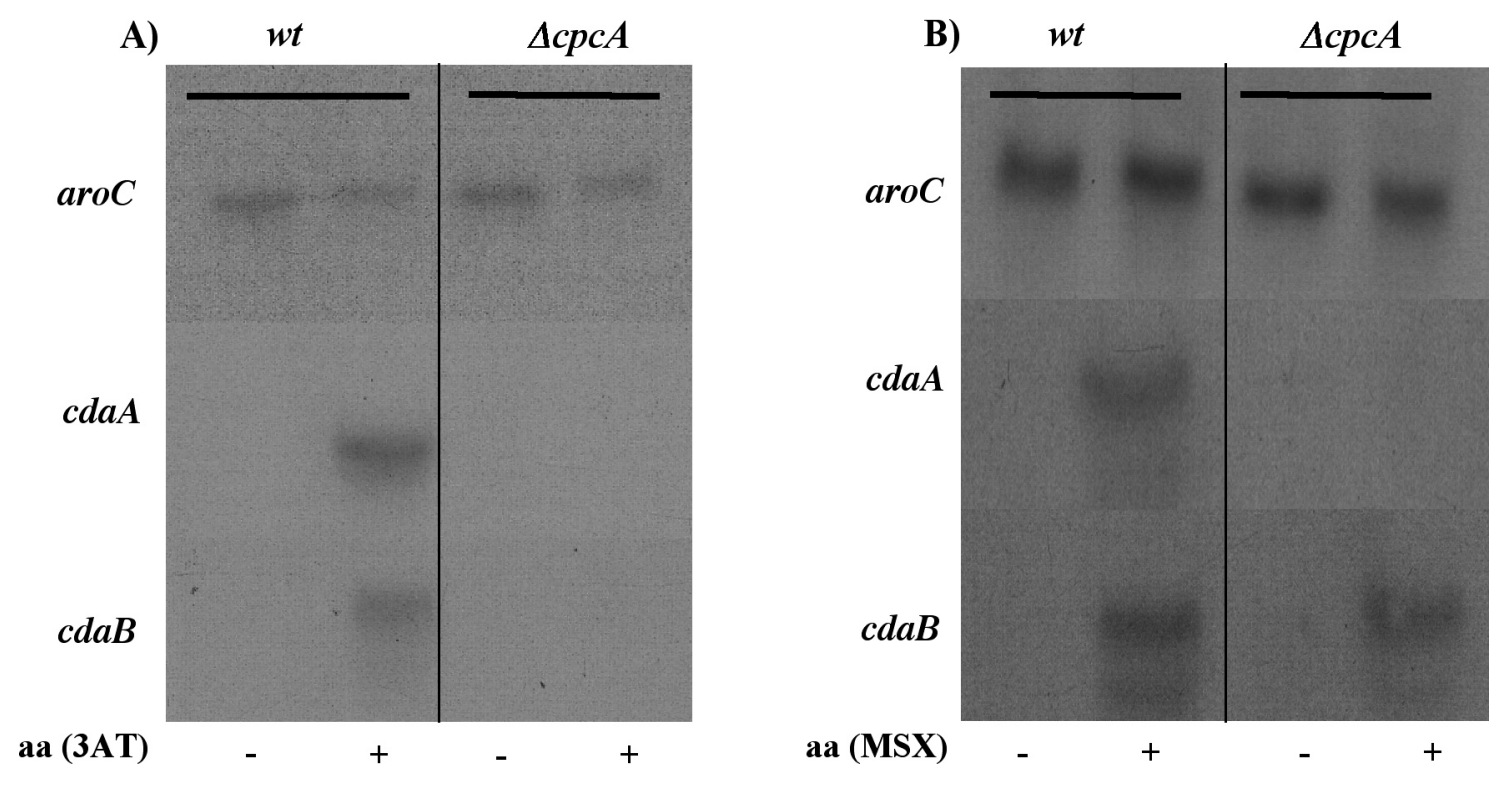

Figure 2.2. Northern hybridisations of the two AAA-ATPases $c d a A$ and $c d a B$ under amino acid starvation A) Induction of both $c d a$ genes under starvation induced by 3AT in the wild-type strain D141. No signal was detected in $c p c A$ deletion strain with and without starvation as well as in the wild-type under non-starvation. B) Transcription of $c d a A$ and $c d a B$ was also increased under amino acid starvation induced by MSX but not under sated conditions; in contrast to $c d a A, c d a B$ appeared also up-regulated in the $\Delta c p c A$ mutant starved for the amino acid glutamine. Accordingly, only for the $c d a A$-encoded gene a CpcA-dependent regulation of transcription was evident. Steady-state levels of the $\operatorname{aroC}$ gene were used as internal standard in the Northern hybridisations.

$\operatorname{cda} A$

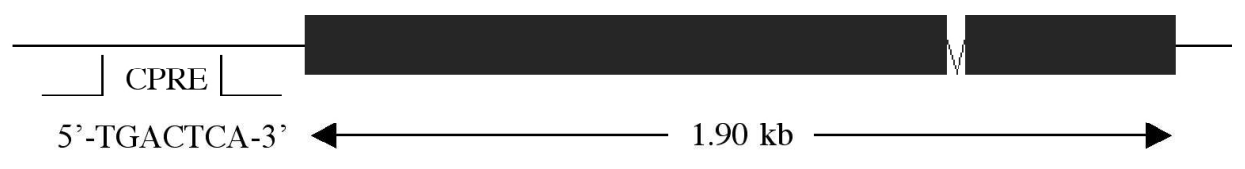

$c d a B$

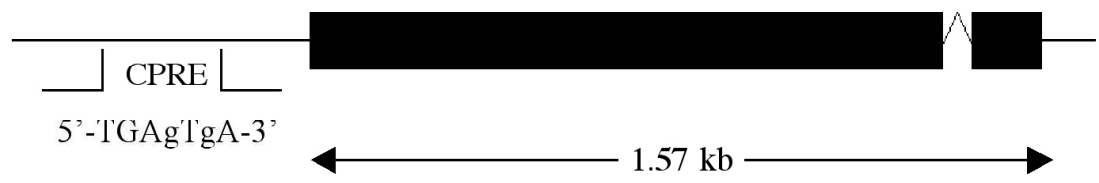

Figure 2.3. $c d a A$ and $c d a B$ with their putative $\mathrm{CpcA}$ binding sites

The figure displays an overview of the two genes $c d a A$ and $c d a B$ with their promoter region. $c d a A$ has a size of $1.90 \mathrm{~kb}$ and contains within $1 \mathrm{~kb}$ of its promoter region the cantoning sequence 5'-TGACTCA-3' as putative CpcA binding region. $c d a B$ is with $1.57 \mathrm{~kb}$ a bit smaller and contains not the high conserved binding region in its promoter. The 5'-TGAgTgA-3' sequence is also a putative recognition site for the $c p c A$ gene product but has a less affinity to this protein than 5'-TGACTCA-3'. 


\subsubsection{Deletion of $c d a A$ and $c d a B$ results in increased sensitivity towards Calcofluor}

\section{White}

To generate a $\Delta c d a A$ deletion strain, a recyclable knock out cassette described by Krappmann et al. (2005) conferring hygromycin resistance was used. To delete the $c d a B$ gene, a comparable construct was used containing a phleomycin resistance (fig. 2.4). Both deletion cassettes were homologously integrated in the D141 wild-type isolate. Moreover, a double knock-out of both genes was made in the D141 background (see Materials and Methods).

$c d a A$ k.o. construct

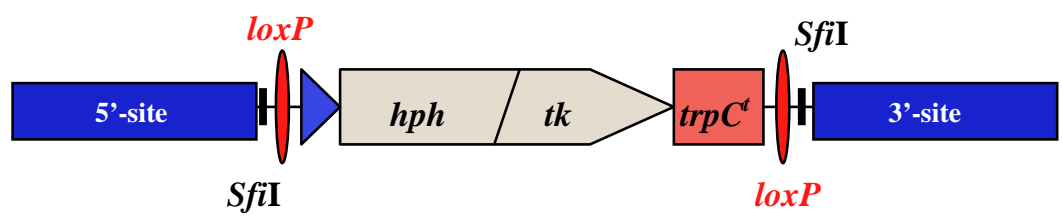

$c d c B$ k.o. construct

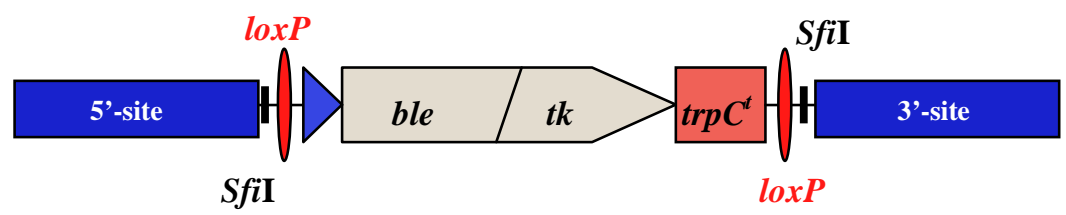

homologous integration in a wt-strain

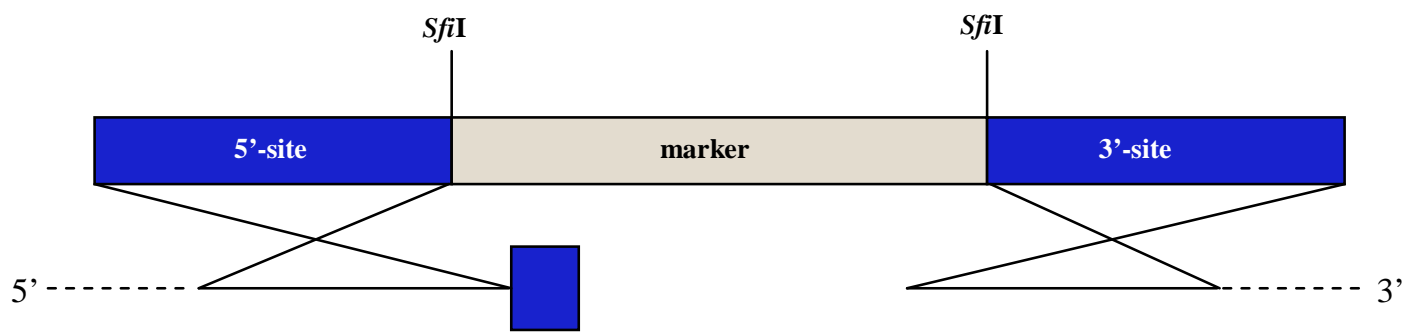

Figure 2.4. Construction of $c d a A, c d a B$ and the $c d a A / B$ deletion strains

For constructing the deletion cassettes we used a hygromycin resistance for the $c d a A$ and a phleomycin resistance for the $c d a B$ gene. Via SfiI restriction sites the 5' and the 3' flanking regions of these genes were connected to the marker cassettes. Via homologous integration the genes were deleted. For counter selection on FUDR the construct contained a thymidin kinase and to make the whole system recyclable there were two loxP sites at the flanking regions of the deletion construct. To create the double deletion $(c d a A / B)$ the $c d a A$ knock-out strain was used.

For the identification of correct deletants, candidates from the transformants' pool were tested by Southern hybridisation. To check for the $c d a A$ deletion, a probe binding to the 5 ' region of the gene was used. Genomic DNA isolated from clonal transformants was digested with the 
enzymes NcoI and PvuII, for comparison wild-type DNA was used. One transformant displaying the expected signals at $4.4 \mathrm{~kb}(\mathrm{NcoI})$ and $5.7 \mathrm{~kb}(P v u \mathrm{II})$ was used for further experiments (fig. 2.5A). To check for deletion of the other $c d a$ gene, a probe binding to the 5', region of $c d a B$ was constructed and used in the Southern hybridisations. Here, the enzymes $S p h \mathrm{I}$ and $S s p \mathrm{I}$ were used for restriction. Signals indicating the correct deletion situation should be at $2.5 \mathrm{~kb}(S p h \mathrm{I})$ and $6.1 \mathrm{~kb}(S s p \mathrm{I})$ (fig. 2.5B). For the double deletion, the $\Delta c d a A$ isolate was used and transformed with the $\Delta c d a B$ replacement cassette. For final validation Southern hybridisation was made with the same probes and enzymes as for the two single knock-outs, and isolates resulting in the correct bands were used for further investigation (fig. 2.5C).

A) $c d a$ A k.o.

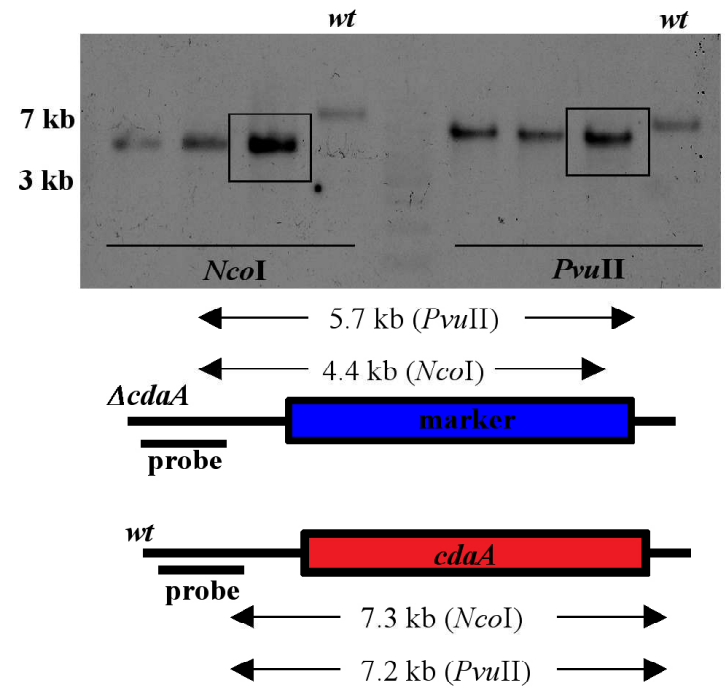

B) $c d a$ B k.o.

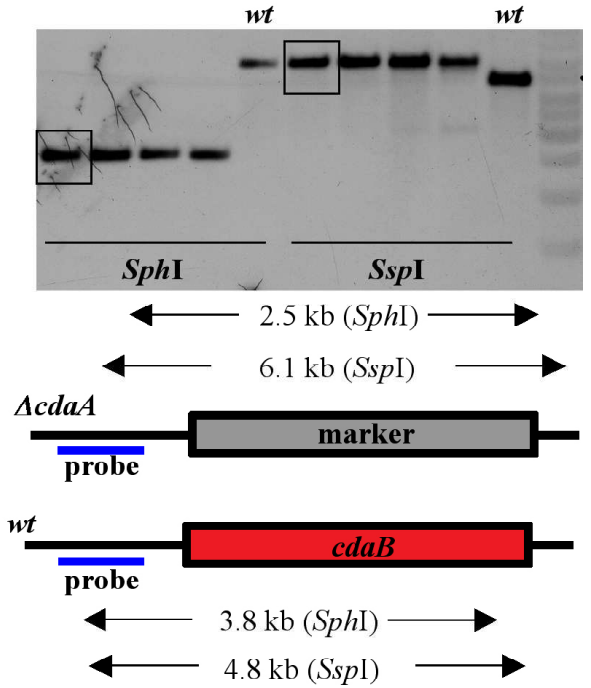

C) $c d a \mathrm{~A} / \mathrm{B}$ k.o.
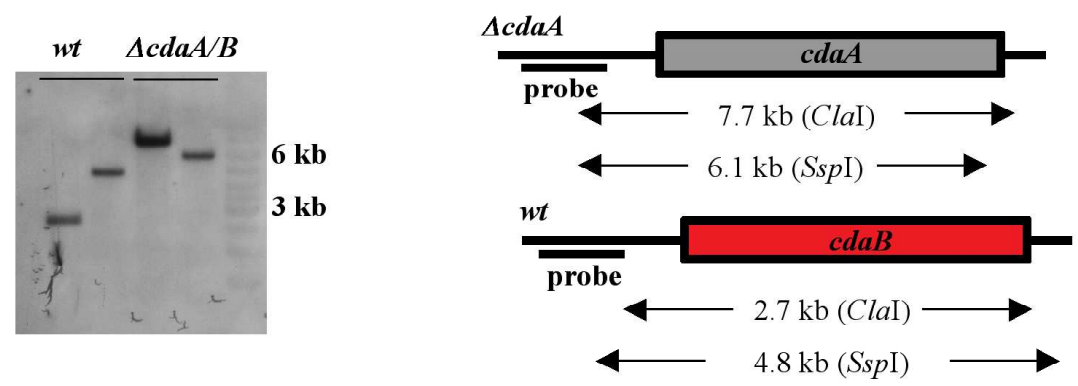

Figure 2.5. Validation of $c d a$ deletions strains by Southern hybridisation

A) Genomic DNA of putative $c d a A$ knock out clone was digested with $N c o$ I and $P v u I I$ resulting in the expected signals at 7.3 and $7.2 \mathrm{~kb}$ by using a probe binding at the $5^{\prime}$ region of $c d a A$. As control the wildtype was used. B) DNA from putative $\Delta c d a B$ strains were digested with $S p h$ I and $S s p$ I resulting in bands with a size of 2.5 and 6.1 $\mathrm{kb}$ for right clones. The prepared probe binds at the $5^{\prime}$ region of $c d a B$. C) Double knock out was made in a $c d a A$ deletion background. Clones were tested by digestion of genomic DNA with ClaI and SspI. Correct deletions lead to signals at 7.7 and $6.1 \mathrm{~kb}$. 
The integrated resistance cassettes in the deletions strains were recycled by transforming a plasmid expressing the Cre recombinase (Krappmann et al., 2005) into the deletion strains. For counterselection purposes, putative clones were plated on FUDR-containing medium (see Materials and Methods), and Southern experiments were executed for validation. To check for recycling of the marker from the $\triangle c d a A$ deletion strain genomic DNA was digested with $N c o I$, and correct clones should show a signal at $5.2 \mathrm{~kb}$. For recycling $\Delta c d a B$ in the single and double deletion strains genomic DNA was digested with $S s p$ I to get a signal at $3.3 \mathrm{~kb}$ for correct clones. Moreover, isolates carrying the deletions of $c d a A$ and $c d a B$ without the corresponding resistance cassette for additional experiments were attempted, but only the phleomycin resistance cassette could be recycled (fig. 2.6A-C).

In additional experiments a set of deletants was tested for growth on different kinds of media. As control and for comparison the wild-type progenitor as well as the cpcA deletion strain were used. On minimal medium no obvious growth phenotype was evident (fig. 2.7A). Under amino acid-limiting conditions using MSX-containing media both $\Delta c d a$ deletion strains grew as well as the wild-type, whereas the $\triangle c p c A$ displayed reduced growth (fig. 2.7B). In contrast to this, all knock out strains displayed sensitivity to Calcofluor White (CW), an inducer of cell wall and septa stress: on medium containing $0.1 \mathrm{mg} / \mathrm{ml} \mathrm{CW}, c d a$ deletion strains and the $\triangle c p c A$ mutant show reduced growth in comparison to the wild-type isolate (fig. 2.7C). Interestingly, the $\Delta c d a A$ mutant carrying the hygromycin resistance cassette displayed a less stringent phenotype in comparison to the $c d a A: \because \operatorname{lox} P$ strain. Accordingly, it can be suggested that the presence of this particular resistance cassette contributes to a certain extent to resistance towards cell wall stress induced by Calcofluor White. This phenomenon was not detectable for the phleomycin resistance in the $\Delta c d a B$ deletion strain or in the $\Delta c d a A$; $c d a B::$ loxP mutant. Based on these results the two AAA-ATPases seem to be involved in cell wall stress resistance. 
A)

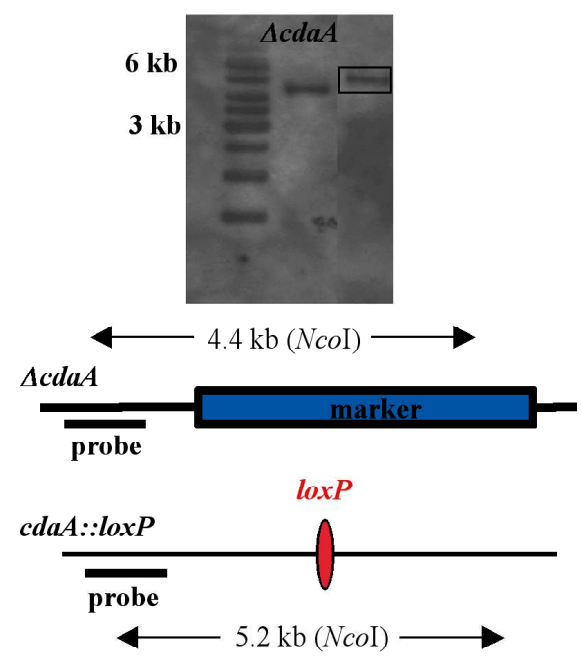

B)

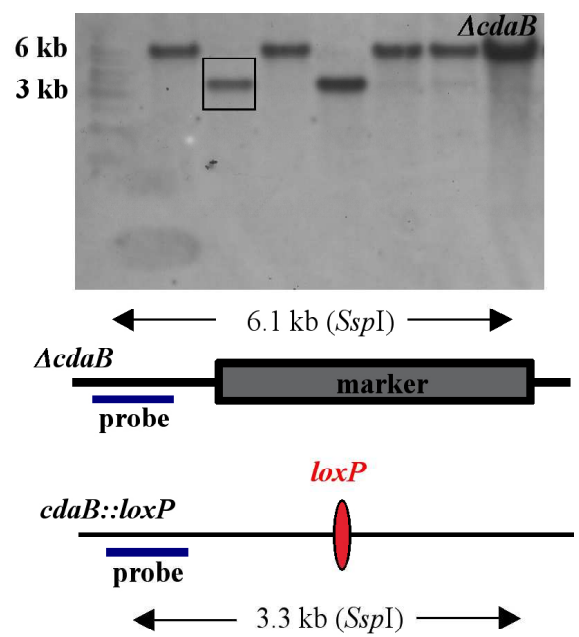

C)

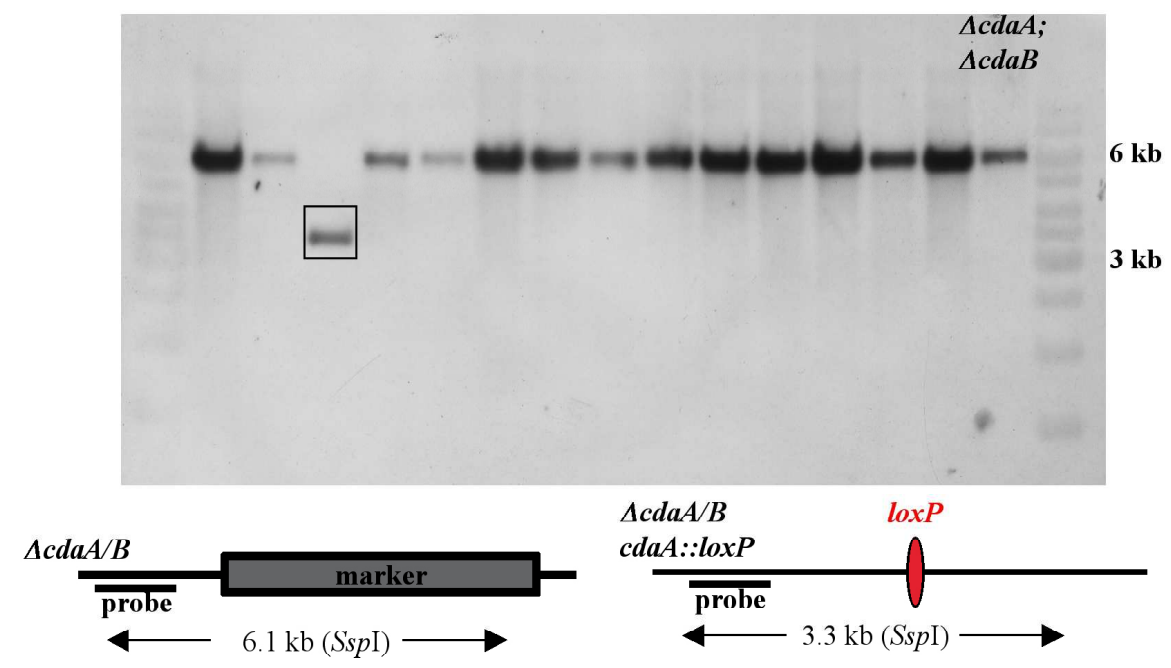

Figure 2.6. Southern hybridisation of the recycled marker systems in $c d a A, c d a B$ and $c d a A ; c d a B$

A) The Southern hybridisation displays a $c d a A$ deletion clone loosing its resistence cassette after transformation with a plasmid containing a Cre recombinase. Digestion was done with $N c o$ I resulting in signals at $5.2 \mathrm{~kb}$ for the right clones. As control we used the $c d a A$ deletion strain with the resistance cassette. B) Digestion with $S s p$ I of different $\Delta c d a B$ clones after recycling the resistance cassette. Correct clones should have the signal at $3.3 \mathrm{~kb}$. As control the $\Delta c d a B$ strain containing the dominant marker was used. C) Southern hybridisation of $c d a A / B$ deletion strains with and without the $c d a B$ marker cassette. Digestion with $S s p$ I leading to a $3.3 \mathrm{~kb}$ signal for the clone without marker. 
A)

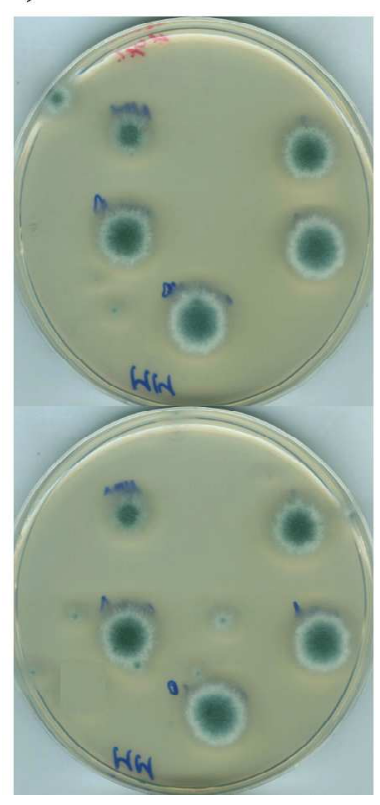

B) MSX 2mM

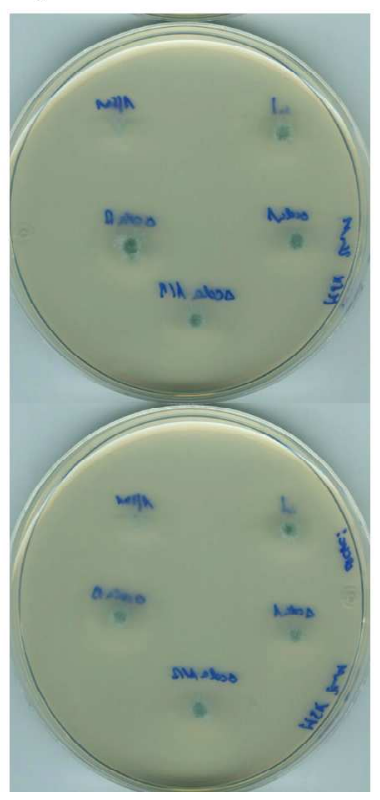

\section{C) $\mathrm{CW} 0.1 \mathrm{mg} / \mathrm{ml}$}

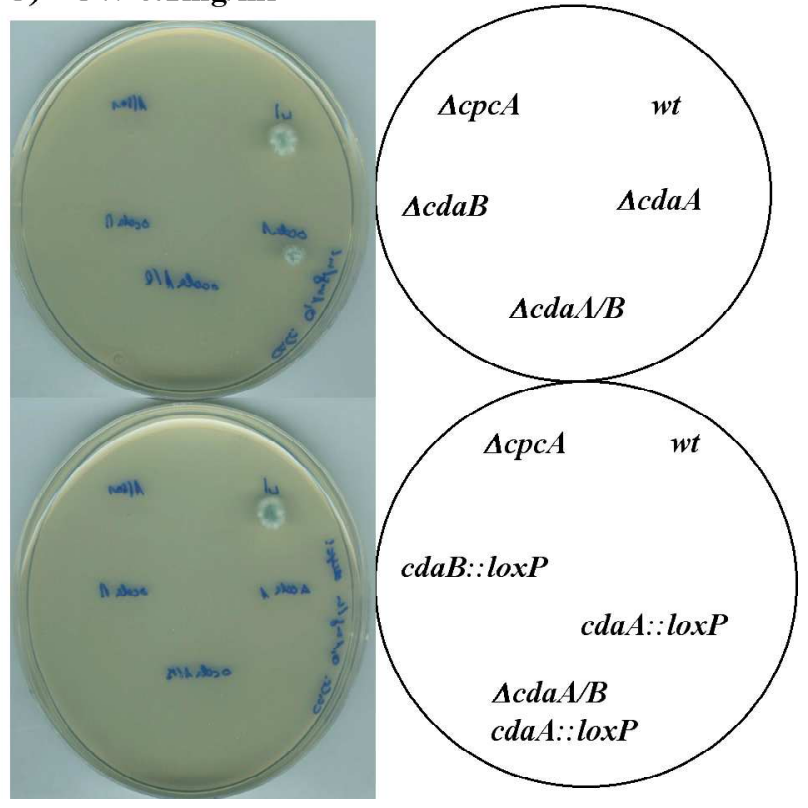

Figure 2.7. Growth test of $c d a$ deletion strains on different media

A) $c d a$ deletions strains with and without marker, the wt and the $\Delta c p c A$ plated on MM. All strains showed a normal growth on this medium. B) Under amino acid starvation by using MSX a phenotype was only visible for the $\Delta c p c A$ strain. The $\Delta c d a$ mutants grew as well as the wild-type. C) On $\mathrm{CW}$ containing medium all mutants showed a growth defect in contrast to the wildtype. Strains having still the hygromycine resistance cassette showed an attenuated phenotype.

\subsubsection{Calcofluor White has no inductive effect on the Cross-Pathway Control}

According to the fact that $\mathrm{CpcA}$ regulates two AAA-ATPases under amino acid starvation and that deletions of these genes lead to an increased sensitivity to Calcofluor White, the question of $\mathrm{CW}$ as CPC-inducing agent was followed. Therefore, the wild-type and the $\Delta c p c A$ deletion strain were inoculated in minimal medium, after $18 \mathrm{~h}$. Afterwards the mycelia were shifted to medium with $0,002 \mathrm{mg} / \mathrm{ml}$ Calcofluor White and incubated for additional $4 \mathrm{~h}$. From these cultures samples for RNA extraction were taken. Northern experiments revealed that transcription of the $c p c A$ gene is slightly induced in the wild-type under stress conditions compared to the untreated sample (fig. 2.8A). RNA samples extracted from cultures induced with a higher concentration of $\mathrm{CW}(0,004 \mathrm{mg} / \mathrm{ml})$ were hybridised with a probe binding to the ornithine carbamoyle transferase-encoding gene $\arg B$, which is regulated by $\mathrm{CpcA}$ and therefore an established indicator for the CPC status. Up-regulation of $\operatorname{argB}$ gene transcription was not evident (fig. 2.8B), indicating that the Cross-Pathway Control is not induced by cell wall stress conditions. 

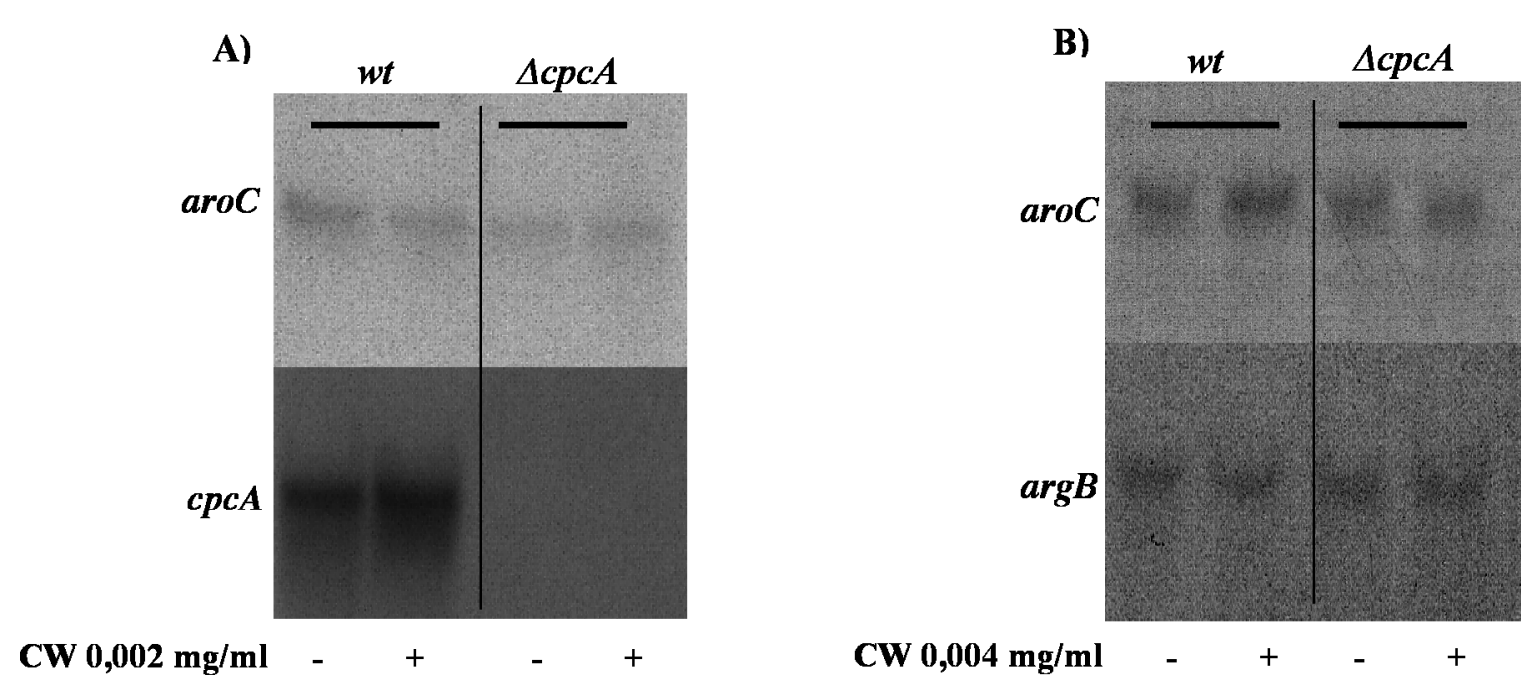

Figure 2.8. Northern hybridisations of the $c p c A$ and the $\arg B$ under Calcofluor White conditions

A) Under cell wall stress by using $0.002 \mathrm{mg} / \mathrm{ml}$ Calcofluor White $(\mathrm{CW})$ is the transcriptional level of $c p c A$ slightly induced in comparison to non-stress conditions. No signal was visible in the $\Delta c p c A$ strain with and without stress. B) Induction with Calcofluor White $(\mathrm{CW})(0.004 \mathrm{mg} / \mathrm{ml}$ does not induce the $\arg B$ gene, an indicator for the induction of the CPC, in the wild-type. No induction was also visible in the $c p c A$ deletion strain. In both Northern experiments the $\mathrm{CpcA}$ independent $\operatorname{aro} C$ gene was used for internal standard. 


\subsection{Discussion}

The Cross-Pathway Control system of filamentous fungi is an adaptive system that enables the organism to react on different kinds of environmental stress. Interestingly, this system is required for full virulence of A. fumigatus (Krappmann et al., 2004). Therefore, the main focus in these studies was to analyse the CPC transcriptome executed by CpcA in the absence and presence of amino acid starvation conditions. These two profiles were compared with a data set generated from the $\triangle c p c A$ deletion strain under starvation and non-starvation conditions with respect to amino acids. Resulting from both transcriptional experiments 523 genes were assigned that appeared to be regulated by the transcriptional regulator CpcA. The majority of them (377) were up-regulated in their transcription and only 146 displayed repression of expression. As expected, many amino acid biosynthesis genes were strongly induced in this study. Especially genes involved in the arginine biosynthesis pathway like the $\arg J$ and the ornithine carbamoylase-encoding $\arg B$ gene were highly induced under amino acid starvation. These results are comparable to the data of $N$. crassa and S. cerevisiae, in which also a high proportion of genes involved in amino acid biosynthetic pathways were derepressed under amino acid starvation conditions (Natarajan et al., 2001; Tian et al., 2007). These genes demonstrate the direct response of the CPC/GC to the internal stress signal. The ribosomal machinery is required to enable a high translation of the amino acid biosynthesis genes. Therefore it is not surprising that three genes which are required for the ribosoms were also up-regulated in our data. The induction of genes encoding ribosomal components of $S$. cerevisisae was also shown by Natarajan et al. (2001). Investigation of the whole data set reveals that a lot of different metabolic pathways are induced under these starvation conditions. From yeast and A. nidulans it is known that other starvation conditions such as glucose deprivation or purine limitation also have an effect on the CPC/GC regulation (Rolfes and Hinnebusch, 1993; Yang et al., 2000). Deduced from the strong diversity of metabolic pathways that are under control of the transcriptional activator $\mathrm{CpcA}$ it becomes clear that the CPC is a global regulator system in fungi like A. fumigatus. We found genes involved in sulfur and carbon metabolism as well as in purine biosynthesis. Also genes of the main catabolic pathways like glycolysis or fatty acid utilisation were detected that show an induction under amino acid starvation. These results and observations leading to the conviction that this stress response system reacts in a non-specific manner thus a lot of genes are activated which are not directly required to engage the stress conditions. Nevertheless, this system enables the fungus to react on many different kinds of stress signals that until now cannot be estimated. The high number of CpcA targets make studies on the CPC a 
challenging task. More than $5 \%$ of all existing genes in A. fumigatus are targets of this regulatory system and its transcriptional regulator. An explanation for this high number of induced genes can be that $\mathrm{CpcA}$ has also the ability to activate other transcriptional regulators (25) leading to a signalling cascade of induced genes. Thus the CPC consists not only of direct targets of $\mathrm{CpcA}$ but comprises also a considerable proportion of indirectly regulated factors.

As mentioned above many different genes are induced under amino acid starvation. In this study it was possible to find not only genes involved in typical metabolism pathways but also in other cellular functions. For instance, a large group of genes encoding transporters and permeases are also increased in their expression under starvation conditions. Some of them are involved in nutrient uptake from the environment. Permeases for amino acids, purines and uracil as well as an oligopeptide transporter were found. It is known from C. albicans and $S$. cerevisisae that oligopeptide transporters are required for taking up small peptides from the environment (Hauser et al., 2001; Reuss and Morschhäuser, 2006). Interestingly, also many transporters that are not involved in nutritional supply were activated under starvation. Most of these transporters belong to the ABC, MSF and MFS multi drug transporter families. Although the function of all these transporters and pumps are not clear, it can be suggested that many of them are involved in a sort of stress or drug response (Andrade et al., 2000a; Andrade et al., 2000b; Del Sorbo et al., 2000; Nakaune et al., 2002; Tobin et al., 1997). Accordingly, the fungus gains the ability to pump toxic molecules coming from the environment out of the cell, by which the organism achieves a higher possibility to survive. Consequently, the CPC is not only a system that enables the fungus to live and grow under limited nutrient conditions but also to react and neutralise toxic molecules.

Furthermore, in the data set genes encoding enzymes with protein degradation function were detected, which may be involved in intracellular turnover of proteins. One of these is an ubiquitin-conjugating enzyme. For this kind of proteins it is known that they are required to tag proteins for subsequent degradation (Muratani and Tansey, 2003). Additionally, some proteins of protease activity were detected, which degrade structures and macromolecules outside the fungus in the near environment to result in freely available resources which then can be assimilated by special transport systems (see above) (Schaal et al., 2007).

The number of CpcA-dependent repressed genes is significant lower than the one of induced ones. This is not surprising since $\mathrm{CpcA}$ is a transcriptional activator. Accordingly, the major effect of repression comes from other transcription factors regulated by $\mathrm{CpcA}$. Most of the repressed targets in the data set are not involved in metabolism required to grow under limited 
conditions: no amino acid biosynthesis genes and only one gene involved in nitrogen metabolism could be found. Interestingly, three transporters required for the iron uptake system were found to be repressed. This implies that the Cross-Pathway Control represses iron uptake and that the involvement of the transcriptional activator $\mathrm{CpcA}$ does not result in positive regulation of the iron transport system, which is essential for virulence of $A$. fumigatus (Schrettl et al., 2004). It also seems to be true for heat tolerance: genes were found to be repressed under amino acid starvation that play a role in a kind of heat stress response. Additional repressed stress factors appeared to a low extent in the transcriptome profiles.

The main focus in this study was set on the detection of positively regulated stress genes, which may be required for virulence of the fungal pathogen. Twelve putative stress genes could be deduced from the data sets. Their possible functions differ from detoxification of reactive oxidative species (ROS) to drug resistance. In addition, two AAA-ATPases with yet unknown function were found. These proteins can affect diverse pathways and mechanisms and are often required in cellular stress response pathways (Hanson and Whiteheart, 2005). By further investigation it was corroborated that both genes are targets of CpcA under amino acid starvation conditions induced by $3 \mathrm{AT}$ and that both genes contain a putative CpcA binding site in their promoter region. This leads to the suggestion that they are direct targets of the transcriptional activator. Further on, both genes were deleted and also a double knockout mutant could be generated with the aim to assign any cellular function to the corresponding gene products. Growth tests of the $c d a$ deletion strains displayed a reduced growth on Calcofluor White-containing medium, which induces cell wall stress for the fungus. In order to confirm the observed phenotypes, further experiments including reconstituted strains have to be carried out. In contrast to this observation, no phenotype could be detected with respect to amino acid starvation for these deletion strains. Also induction of the CPC system under cell wall stress induced by CW treatment failed. Interestingly, slight induction of the $\mathrm{CpcA}$ enconding gene was detected under this kind of stress condition. From these preliminary data it may be deduced that the $c p c A$ gene can be induced independently from the Cross-Pathway Control. This is supported by the transcriptional profile of $A$. fumigatus under antifungal drug conditions using voriconazole as published by da Silva Ferreira et al. (2006), where cpcA expression was also induced but typical genes for amino acid biosynthesis were not regulated.

In summary it can be stated that the CPC is a global regulatory system that affect more than 500 genes in order to give the fungus the ability to react on many different kinds of stress and starvation. Moreover, expression of the transcriptional activator $\mathrm{CpcA}$, an integral part of this 
regulatory system can be induced independently from amino acid starvation conditions, which are the prime stress conditions to trigger the Cross-Pathway Control in fungi. 


\subsection{References}

Andrade, A.C., Del Sorbo, G., Van Nistelrooy, J.G., and Waard, M.A. (2000a) The ABC transporter AtrB from Aspergillus nidulans mediates resistance to all major classes of fungicides and some natural toxic compounds. Microbiology 146 (Pt 8): 1987-1997.

Andrade, A.C., Van Nistelrooy, J.G., Peery, R.B., Skatrud, P.L., and De Waard, M.A. (2000b) The role of $\mathrm{ABC}$ transporters from Aspergillus nidulans in protection against cytotoxic agents and in antibiotic production. Mol Gen Genet 263: 966-977.

Brown, T., Mackey, K. (1997) Analysis of RNA by Northern and slot blot hybridization. . In: Current Protocols in Molecular Biology. John Wiley \& Sons Inc.: 4.9.1-4.9.16.

Burns, C., Geraghty, R., Neville, C., Murphy, A., Kavanagh, K., and Doyle, S. (2005) Identification, cloning, and functional expression of three glutathione transferase genes from Aspergillus fumigatus. Fungal Genet Biol 42: 319-327.

Carsiotis, M., Jones, R.F., and Wesseling, A.C. (1974) Cross-pathway regulation: histidinemediated control of histidine, tryptophan, and arginine biosynthetic enzymes in Neurospora crassa. J Bacteriol 119: 893-898.

Chang, Y.C., Tsai, H.F., Karos, M., and Kwon-Chung, K.J. (2004) THTA, a thermotolerance gene of Aspergillus fumigatus. Fungal Genet Biol 41: 888-896.

da Silva Ferreira, M.E., Malavazi, I., Savoldi, M., Brakhage, A.A., Goldman, M.H., Kim, H.S., Nierman, W.C., and Goldman, G.H. (2006) Transcriptome analysis of Aspergillus fumigatus exposed to voriconazole. Curr Genet 50: 32-44.

Davis, R.H., (2000) Neurospora: Contributions of a Model Organism. Oxford, England: Oxford University Press.

Del Sorbo G., Schoonbeek, H., De Waard M.A. (2000) Fungal transporters involved in efflux of natural toxic compounds and fungicides. Fungal Genet Biol 30: 1-15.

Delforge, J., Messenguy, F., and Wiame, J.M. (1975) The regulation of arginine biosynthesis in Saccharomyces cerevisiae. The specificity of argR-mutations and the general control of amino-acid biosynthesis. Eur J Biochem 57: 231-239.

Denning, D.W., Venkateswarlu, K., Oakley, K.L., Anderson, M.J., Manning, N.J., Stevens, D.A., Warnock, D.W., and Kelly, S.L. (1997) Itraconazole resistance in Aspergillus fumigatus. Antimicrob. Agents Chemother 41: 1364-1368.

Denning, D.W., Radford, S.A., Oakley, K.L., Hall, L., Johnson, E.M., and, Warnock, D.W. (1997) Correlation between in vitro susceptibility testing to itraconazole and in vivo outcome of Aspergillus fumigatus infection. J Antimicrob Chemother 40: 401-414.

Diaz-Guerra, T.M., Mellado, E., Cuenca-Estrella, M., and Rodriguez-Tudela, J.L. (2003) A point mutation in the $14 \alpha$-sterol demethylase gene cyp51A contributes to itraconazole resistance in Aspergillus fumigatus. Antimicrob Agents Chemother 47: 1120-1124.

Ellis, M. (1999) Therapy of Aspergillus fumigatus-related diseases. Contrib Microbiol 2: 105129.

Espinel-Ingroff, A., Fothergill, A., Ghannoum, M., Manavathu, E., Ostrosky-Zeichner, L., Pfaller, M., Rinaldi, M., Schell, W., and Walsh, T. (2005) Quality control and reference guidelines for CLSI broth microdilution susceptibility method (M 38-A document) for amphotericin $\mathrm{B}$, itraconazole, posaconazole, and voriconazole. J Clin Microbiol 43: 5243-5246.

Feinberg, A.P., Vogelstein, B. (1983) A technique for radiolabeling DNA restriction endonuclease fragments to high specific activity. Anal Biochem 132: 6-13.

Han, K.H., Seo, J.A., and Yu, J.H. (2004) Regulators of G-protein signalling in Aspergillus nidulans: RgsA downregulates stress response and stimulates asexual sporulation through attenuation of GanB (Galpha) signalling. Mol Microbiol 53: 529-540.

Hanson, P.I., and Whiteheart, S.W. (2005) AAA+ proteins: have engine, will work. Nat Rev Mol Cell Biol 6: 519-529. 
Hauser, M., Narita, V., Donhardt, A.M., Naider, F., and Becker, J.M. (2001) Multiplicity and regulation of genes encoding peptide transporters in Saccharomyces cerevisiae. Mol Membr Biol 18: 105-112.

Hensel, M., Arst, H.N., Jr., Aufauvre-Brown, A., and Holden, D.W. (1998) The role of the Aspergillus fumigatus areA gene in invasive pulmonary aspergillosis. Mol Gen Genet 258: 553-557.

Higa, A., and Mandel, M. (1970) Actinomycin sensitive mutants of Escherichia coli K-12. Mol Gen Genet 108: 41-46.

Hinnebusch, A.G. (2005) Translational regulation of GCN4 and the general amino acid control of yeast. Annu Rev Microbiol 59: 407-450.

Hoffmann, B., Valerius, O., Andermann, M., and Braus, G.H. (2001) Transcriptional autoregulation and inhibition of mRNA translation of amino acid regulator gene $c p c A$ of filamentous fungus Aspergillus nidulans. Mol Biol Cell 12: 2846-2857.

Käfer, E. (1977) Meiotic and mitotic recombination in Aspergillus nidulans and its chromosomal aberrations. Adv Genet 19: 131-133.

Kolar, M., Punt, P.J., van den Hondel, C.A., and Schwab, H. (1988) Transformation of Penicillium chrysogenum using dominant selection markers and expression of an Escherichia coli lacZ fusion gene. Gene 62: 127-134.

Krappmann, S., Bignell, E.M., Reichard, U., Rogers, T., Haynes, K., and Braus, G.H. (2004) The Aspergillus fumigatus transcriptional activator $\mathrm{CpcA}$ contributes significantly to the virulence of this fungal pathogen. Mol Microbiol 52: 785-799.

Krappmann, S., Bayram, O., and Braus, G.H. (2005) Deletion and allelic exchange of the Aspergillus fumigatus veA locus via a novel recyclable marker module. Eukaryot Cell 4: $1298-1307$.

Kumanovics, A., Poruk, K.E., Osborn, K.A., Ward, D.M., and Kaplan, J. (2006) YKE4 (YIL023C) encodes a bidirectional zinc transporter in the endoplasmic reticulum of Saccharomyces cerevisiae. J Biol Chem 281: 22566-22574.

Latgé, J.P. (1999) Aspergillus fumigatus and aspergillosis. Clin Microbiol Rev 12: 310-350.

Lee, B.N., and Adams, T.H. (1994) Overexpression of flbA, an early regulator of Aspergillus asexual sporulation, leads to activation of $\operatorname{brl} A$ and premature initiation of development. Mol Microbiol 14: 323-334.

Meletiadis, J., Antachopoulos, C., Stergiopoulou, T., Pournaras, S., Roilides, E., and Walsh, T.J. (2007) Differential fungicidal activities of amphotericin B and voriconazole against Aspergillus species determined by microbroth methodology. Antimicrob Agents Chemother 51: 3329-3337.

Millner, P.D., Marsh, P.B., Snowden, R.B., and Parr, J.F. (1977) Occurrence of Aspergillus fumigatus during composting of sewage sludge. Appl Environ Microbiol 34: 765-772.

Muratani, M., and Tansey, W.P. (2003) How the ubiquitinproteasome system controls transcription. Nature Rev Mol Cell Biol 4: 1-10.

Nakaune, R., Hamamoto, H., Imada, J., Akutsu, K., and Hibi, T. (2002) A novel ABC transporter gene, PMR5, is involved in multidrug resistance in the phytopathogenic fungus Penicillium digitatum. Mol Genet Genomics 267: 179-185.

Natarajan, K., Meyer, M.R., Jackson, B.M., Slade, D., Roberts, C., Hinnebusch, A.G., and Marton, M.J. (2001) Transcriptional profiling shows that Gen4p is a master regulator of gene expression during amino acid starvation in yeast. Mol Cell Biol 21: 43474368.

Panepinto, J.C., Oliver, B.G., Amlung, T.W., Askew, D.S., and Rhodes, J.C. (2002) Expression of the Aspergillus fumigatus rheb homologue, rhbA, is induced by nitrogen starvation. Fungal Genet Biol 36: 207-214. 
Panepinto, J.C., Oliver, B.G., Fortwendel, J.R., Smith, D.L., Askew, D.S., and Rhodes, J.C. (2003) Deletion of the Aspergillus fumigatus gene encoding the Ras-related protein RhbA reduces virulence in a model of Invasive pulmonary aspergillosis. Infect Immun 71: 2819-2826.

Piotrowska, M. (1980) Cross-Pathway regulation of ornithine carbamoyltransferase synthesis in Aspergillus nidulans. J Gen Microbiol 116: 335-339.

Punt, P.J., and van den Hondel, C.A. (1992) Transformation of filamentous fungi based on hygromycin B and phleomycin resistance markers. Methods Enzymol 216: 447-457.

Reuss, O., and Morschhäuser, J. (2006) A family of oligopeptide transporters is required for growth of Candida albicans on proteins. Mol Microbiol 60: 795-812.

Rolfes, R.J., and Hinnebusch, A.G. (1993) Translation of the yeast transcriptional activator GCN4 is stimulated by purine limitation: implications for activation of the protein kinase GCN2. Mol Cell Biol 13: 5099-5111.

Ruijter, G.J., and Visser, J. (1997) Carbon repression in Aspergilli. FEMS Microbiol Lett 151: 103.

Sachs, M.S. (1996) General and cross-pathway controls of amino acid biosynthesis. In: The Mycota: Biochemistry and Molecular Biology, ed. R. Brambl and G.A., Marzluf Heidelberg, Germany: Springer Verlag III: 315-345.

Saiki, R.K., Scharf, S., Faloona, F., Mullis, K.B., Horn, G.T., Erlich, H.A., and Arnheim, N. (1985) Enzymatic amplification of beta-globin genomic sequences and restriction site analysis for diagnosis of sickle cell anemia. Science 230: 1350-1354.

Schaal, R., Kupfahl, C., Buchheidt, D., Neumaier, M., and Findeisen, P. (2007) Systematic identification of substrates for profiling of secreted proteases from Aspergillus species. J Microbiol Methods 71: 93-100.

Schrettl, M., Bignell, E., Kragl, C., Joechl, C., Rogers, T., Arst, H.N., Jr., Haynes, K., and Haas, H. (2004) Siderophore biosynthesis but not reductive iron assimilation is essential for Aspergillus fumigatus virulence. J Exp Med 200: 1213-1219.

Schurch, A., Miozzari, J., and Hutter, R. (1974) Regulation of tryptophan biosynthesis in Saccharomyces cerevisiae: mode of action of 5-methyl-tryptophan and 5-methyltryptophan-sensitive mutants. J Bacteriol 117: 1131-1140.

Southern, E.M. (1975) Detection of specific sequences among DNA fragments separated by gel electrophoresis. J Mol Biol 98: 503-517.

Staib, F., Mishra, S.K., Rajendran, C., Voigt, R., Steffen, J., Neumann, K.H., Hartmann, C.A., and Heins, G. (1980) A notable Aspergillus from a mortal aspergilloma of the lung. New aspects of the epidemiology, serodiagnosis and taxonomy of Aspergillus fumigatus. Zentralbl Bakteriol A 247: 530-536.

Tian, C., Kasuga, T., Sachs, M.S., and Glass, N.L. (2007) Transcriptional Profiling of Cross Pathway Control in Neurospora crassa and Comparative Analysis of the Gcn4 and CPC1 Regulons. Eukaryot Cell 6: 1018-1029.

Tobin, M.B., Peery, R.B., and Skatrud, P.L. (1997) Genes encoding multiple drug resistancelike proteins in Aspergillus fumigatus and Aspergillus flavus. Gene 200: 11-23.

van Heerden, I., Cronjé C., Swart S.H., Kotzé J.M. (2002) Microbial, chemical and physical aspects of citrus waste composting. Bioresour Technol. 81: 71-76.

Vermitsky, J.P., Earhart, K.D., Smith, W.L., Homayouni, R., Edlind, T.D., and Rogers, P.D. (2006) Pdr1 regulates multidrug resistance in Candida glabrata: gene disruption and genome-wide expression studies. Mol Microbiol 61: 704-722.

Wolfner, M., Yep, D., Messenguy, F., and Fink, G.R. (1975) Integration of amino acid biosynthesis into the cell cycle of Saccharomyces cerevisiae. J Mol Biol 96: 273-290. 
Woodcock, D.M., Crowther, P.J., Doherty, J., Jefferson, S., DeCruz, E., Noyer-Weidner, M., Smith, S.S., Michael, M.Z., and Graham, M.W. (1989) Quantitative evaluation of Escherichia coli host strains for tolerance to cytosine methylation in plasmid and phage recombinants. Nucleic Acids Res 17: 3469-3478.

Yang, R., Wek, S.A., and Wek, R.C. (2000) Glucose limitation induces GCN4 translation by activation of Gcn2 protein kinase. Mol Cell Biol 20: 2706-2717.

$\mathrm{Yu}$, J.H., Wieser, J., and Adams, T.H. (1996) The Aspergillus FlbA RGS domain protein antagonizes $\mathrm{G}$ protein signaling to block proliferation and allow development. Embo $J$ 15: 5184-5190. 
Chapter 3

\section{Basal Expression of the Aspergillus fumigatus Transcriptional Activator CpcA is Sufficient to Support Pulmonary Aspergillosis}

\subsection{Abstract}

Aspergillosis is a disease determined by various factors that influence fungal growth and fitness. A conserved signal transduction cascade linking environmental stress to amino acid homeostasis is the Cross-Pathway Control (CPC) system that acts via phosphorylation of the translation initiation factor eIF2 by a sensor kinase to elevate expression of a transcription factor. Ingestion of Aspergillus fumigatus conidia by macrophages does not trigger this stress response, suggesting that their phagosomal microenvironment is not deficient in amino acids. The $с р с C$ gene encodes the CPC eIF2 $\alpha$ kinase, and deletion mutants show increased sensitivity towards amino acid starvation. $\mathrm{CpcC}$ is specifically required for the $\mathrm{CPC}$ response but has limited influence on the amount of phosphorylated eIF2 $\alpha$. Strains deleted for the $c p c C$ locus are not impaired in virulence in a murine model of pulmonary aspergillosis. Accordingly, basal expression of the Cross-Pathway Control transcriptional activator appears sufficient to support aspergillosis in this disease model. 


\subsection{Introduction}

Besides bacteria, fungal species represent the second-biggest group of pathogens that are able to infect humans (Woolhouse, 2006), and therefore it is of future interest to elucidate the mechanisms of disease caused by fungal pathogens thoroughly. Pathogenicity often is a multifactorial trait composed of a variety of factors that contribute to the virulence of a fungal organism (Casadevall, 2006). Characteristics of this so-called virulome determine the outcome of a pathogen-host encounter in strict dependency of the host's immune status, as it has comprehensively been depicted by the damage-response framework (Casadevall and Pirofski, 2003).

Within this concept, fungi of the genus Aspergillus represent a distinct category as they can harm an infected individual by eliciting a strong, allergic immune response, or when the major defence lines of innate immunity are impaired. Especially the latter scenario may result in severe and fatal forms of so-called aspergillosis, which are characterised by invasive and disseminated progressions. The predominant species to cause aspergillosis is the deuteromycete Aspergillus fumigatus, but also other aspergilli have been reported in recent studies to cause this disease (Brakhage, 2005; Walsh and Groll, 2001). In general, Aspergillus inhabits organic substrates in decaying matters to lead a saprobic lifestyle, and based on the hypothesis that selective environmental pressure forms a facultative pathogenic fungus (Casadevall et al., 2003), this ecological niche is likely to have shaped the virulence of A. fumigatus. Accordingly, thermophyly, stress resistance, and nutritional versatility are crucial for Aspergillus pathogenicity as they support survival and propagation in an alternative ecological niche, the human host.

In eukaryotes, resistance against environmental stress conditions is conferred in part by a well-conserved signal transduction cascade that acts on the level of protein synthesis. In this integrated stress response network, diverse conditions of stress are perceived by various kinases that phosphorylate the $\alpha$ subunit of an initiation factor for translation, eIF2 (Harding et al., 2003; Wek et al., 2006). This in turn lowers cellular rates of translation initiation except for distinct mRNAs that code for regulatory molecules like transcription factors. Consequently, expression of such a terminal effector is increased to generate a cellular counter-reaction to the triggering stress condition. Higher eukaryotes express up to four types of eIF2 $\alpha$ kinases, each responding to distinct signals, whereas in the model ascomycete Saccharomyces cerevisiae only one such sensor kinase, Gcn2p, has been identified (Wek et $a l .$, 1995). The primary signal Gen2p responds to is amino acid starvation, and the regulatory network constituted by this eIF2 $\alpha$ kinase and its effector Gcn $4 p$ has been coined as General 
Control (GC) of amino acid biosynthesis. In filamentous fungi, as Neurospora crassa or Aspergillus spp., the homologous system is called Cross-Pathway Control (CPC) (Carsiotis and Jones, 1974; Carsiotis et al., 1974; Hoffmann et al., 2001; Wanke et al., 1997). In general, binding of accumulated uncharged tRNA molecules, that mirror amino acid starvation, to the GC/CPC sensor kinase Gen2p results in activation of an intrinsic protein kinase activity to phosphorylate the $\alpha$ subunit of the trimeric eIF2 complex at a highly conserved serine residue (Ser-51). As a result, translation of a transcriptional activator, $\mathrm{Gcn} 4 \mathrm{p} / \mathrm{CpcA}$, is elevated and generates a global cellular response. Characterisation of the corresponding transcriptional effector in A. fumigatus, the cpcA gene product, has led to several conclusions: first, $\mathrm{CpcA}$ is a functional orthologue of yeast Gcn4p; second, it is strictly required for the CPC response upon amino acid starvation conditions; third, mutants of A. fumigatus deleted for the coding sequence are attenuated in virulence, as monitored in an animal model for pulmonary aspergillosis using neutropenic mice, but show no obvious growth phenotype in vitro (Krappmann et al., 2004).

As regulation of $\mathrm{CpcA}$ expression in A. fumigatus is likely to be affected by an upstream eIF2 $\alpha$ kinase and may be accordingly complex, the precise role of this transcriptional activator as a virulence determinant remains to be specified. To address this issue and to answer the question whether a de-repressed CPC system is required for aspergillosis, we aimed at the A. fumigatus CPC eIF2 $\alpha$ kinase CpcC. Mutant strains lacking the $\operatorname{cpcC}$ gene product were generated and characterised with respect to amino acid starvation conditions, the CPC response, and virulence. 


\subsection{Materials and Methods}

\subsubsection{Strains, media, and growth conditions}

Bacterial strains were Escherichia coli DH5 $\alpha$ [F', F80d/lacZM15, A(lacZYA-argF)U169, recA1, endA1, hsdR17 ( $\left.r_{K^{-}}, m_{K_{+}}\right)$, supE44, $\lambda^{-}$, thil, gyrA96, relA1] (Woodcock et al., 1989) for general cloning procedures and $\mathrm{KS} 272\left[\mathrm{~F}^{-}, \Delta l a c X 74\right.$, galE, galK, thil, rpsL, $\triangle p h o A(P v u I I)]$ carrying the pKOBEG plasmid for recombineering (Chaveroche et al., 2000). E. coli strains were propagated in LB or LBLS (1\% bacto-tryptone, $0.5 \%$ yeast extract, $1 \%$ or $0.5 \% \mathrm{NaCl}, \mathrm{pH} 7.5)$ medium. Fungal strains used in this study are listed in table 3.1. Growth of A.fumigatus strains was carried out at $37^{\circ} \mathrm{C}$ on minimal medium prepared and supplemented according to (Käfer, 1977). Antibiotics' concentrations were $100 \mu \mathrm{g} / \mathrm{ml}$ for ampicillin, $25 \mu \mathrm{g} / \mathrm{ml}$ for chloramphenicol, and $20 \mu \mathrm{g} / \mathrm{ml}$ for phleomycin.

\subsubsection{Transformation procedures}

Protocols for E. coli were either for calcium/manganese-treated cells (Hanahan et al., 1991) or for electroporation (Dower et al., 1988) with a BIORAD GenePulser at $2.5 \mathrm{kV}$ in $0.2 \mathrm{~cm}$ cuvettes. A. fumigatus was transformed by polyethylene glycol-mediated fusion of protoplasts as described (Punt and van den Hondel, 1992).

\subsubsection{Manipulation of nucleic acids and plasmid constructions}

Standard protocols of recombinant DNA technology were carried out (Sambrook et al., 1989). Pfu proofreading polymerase was generally used in polymerase chain reactions (Saiki et al., 1986) and essential cloning steps were verified by sequencing on an ABI PRISM 310 capillary sequencer. Fungal genomic DNA was prepared according to Kolar et al. (Kolar et al., 1988), and Southern analyses were carried out essentially as described (Southern, 1975). Total RNA samples were isolated employing the TRIzol reagent of INVITROGEN followed by Northern hybridisation according to the protocols cited by Brown and Mackey (Brown and Mackey, 1997). Random primed labelling was performed with the STRATAGENE PRIME-IT ${ }^{\circledR}$ II kit in the presence of $\left[\alpha_{-}{ }^{32} \mathrm{P}\right]$-dATP (Feinberg and Vogelstein, 1983). Autoradiographies were produced by exposing the washed membranes to KODAK X-OMAT films.

Plasmids used and constructed during the course of this study are listed and briefly described in table 1, together with essential oligonucleotides employed to construct them. pME3286 served as reference for the $c p c C$ sequence and contains a genomic DraI/NaeI fragment cloned in the EcoRV site of the general cloning plasmid pGEM5(+). To construct a deletion cassette for the $c p c C$ locus, a suitable template was constructed as follows: a $3.1 \mathrm{~kb} \mathrm{Fsp} /$ SpeI 
fragment comprising the $\operatorname{cpcC} 5$ ' region was inserted into pGEM5(+) via EcoRV/SpeI, followed by insertion of a 3' PstI fragment $(5.1 \mathrm{~kb})$ into the Mph1103I site. The resulting plasmid pME3287 was digested with NdeI and NotI, and the resulting backbone was cotransformed with a PCR amplicon from pME2891 with primers Sv117/118 into the E. coli recipient KS272 replicating pKOBEG (Chaveroche et al., 2000). The resulting construct from this recombineering step is pME3288, which served as replacement cassette for the $c p c C$ coding sequence. For $\mathrm{N}$-terminal tagging of the $c p c A$ coding region, the $g f p 2-5$ sequence was amplified with primer pair CS41/CS42 to become inserted after SalI/XhoI digestion into the SalI site of pME2563, yielding pME3289. From this construct, a $9 \mathrm{~kb}$ fragment was released via BssHII to reconstitute the $c p c A$ locus in deletion strain AfS01.

For determination of the actual $c p c C$ coding sequence, reverse transcription from an oligo(dT) primer (MBI FERMENTAS) was carried out on total RNA that had been isolated from vegetative D141 cultures, pairs of oligonucleotides (Sv319 - Sv336) were used to amplify suitable stretches spanning predicted intronic regions from this template, and the resulting cDNA amplicons were directly sequenced.

\subsubsection{Biochemical methods and Western blots}

Crude extracts were prepared by grinding washed and shock-frozen mycelia to a fine powder and extracting soluble proteins with buffer $(100 \mathrm{mM}$ Tris. $\mathrm{HCl}, 200 \mathrm{mM} \mathrm{NaCl}, 20 \%$ glycerol, $5 \mathrm{mM}$ EDTA, $\mathrm{pH}$ 8) at $4^{\circ} \mathrm{C}$ in the presence of the protease inhibitor phenylmethylsulfonylflouride. Protein contents were determined by the procedure of Bradford (Bradford, 1976). Enzymatic activities of ornithine carbamoyltransferase (OTCase, E.C. 2.1.33) activities were determined according to (Tian et al., 1994). Western analysis of eIF2 $\alpha$ phosphorylation was carried out essentially as described earlier (Grundmann et al., 2001) with a polyclonal anti-eIF2 $\alpha$ antibody (provided by Alan Hinnebusch; (Romano et al., 1998) and a phosphorylation-specific polyclonal anti-eIF2 $\alpha \sim \mathrm{P}$ antibody (BIOSOURCE International, Camarillo, CA, USA). Cross-reactions were visualised using the ECL technology (AMERSHAM PHARMACIA Biotech).

\subsubsection{Virulence tests in a murine model of pulmonary aspergillosis}

Outbred male mice (strain CD1, 20-28 g, Charles Rivers Breeders) were used for animal experiments. Immunosuppression was executed with hydrocortisone acetate $(112 \mathrm{mg} / \mathrm{kg}$ subcutaneous) and cyclophosphamide (150 mg/kg intraperitoneal) following the protocol of Smith et al. (1994), bacterial infections were prevented by adding tetracycline (1 g/l) and 
ciproxin $(64 \mathrm{mg} / \mathrm{l})$ to the drinking water. Inocula of up to $2 \times 10^{5}$ conidiospores in $40 \mu 1$ of saline were prepared by harvesting spores from 5-day-old slants of solid medium followed by filtration through miracloth and washing with saline (Aufauvre-Brown et al., 1998; Tang et $a l .$, 1993). Mice were anaesthetized by inhalation of halothane and infected by intranasal instillation. The weights of infected and control animals were monitored for up to 10 days twice daily and mice developing severe pulmonary illness, characterized by respiratory distress, hunched posture and poor mobility, or $20 \%$ weight loss were culled. Lungs of these animals were homogenized in saline and aliquots were spread on standard medium to check for fungal growth.

\subsubsection{Cell preparations}

Cells for confrontation assays were essentially prepared as described by Gunzer and coworkers (Behnsen et al., 2007): murine alveolar macrophages were obtained by washing the trachea and lungs of $\mathrm{BALB} / \mathrm{c}$ mice with $\mathrm{PBS}$ through a $22 \mathrm{G}$ plastic catheter to obtain bronchoalveolar lavage fluid. After erythrocyte lysis, the cells were resuspended in complete medium supplemented with glutamine, penicillin, and streptomycin and kept on ice until further use; J774 cells were cultured in BioWhittaker's X-Vivo 15 medium.

\subsubsection{Fluorescence and light microscopy}

Conidia and mycelia were examined with a Zeiss Axiovert microscope by either differential interference contrast microscopy (DIC) or fluorescence microscopy using a GFP filter set (AHF Analysentechnik AG, Tübingen, Germany). Photographs were taken using a Xillix Microimager digital camera and the Improvision Openlab software (IMPROVISION, Coventry, UK).

Table 3.1. Strains, plasmids and oligonucleotides used in this study

\begin{tabular}{|l|l|}
\hline Name & Description or sequence \\
\hline Strains & \\
\hline D141 & Aspergillus fumigatus wild-type strain (syn. NRRL 6585), clinical isolate \\
\hline AfS01 & strain D141 carrying deletion of $c p c A$ coding sequence: Phleo $^{\mathrm{r}}, \mathrm{MSX}^{\mathrm{s}}$ \\
\hline AfS26 & strain D141 carrying deletion of $c p c C$ coding sequence: Phleo $^{\mathrm{r}}, \mathrm{MSX}^{\mathrm{s}}$ \\
\hline AfS27 & AfS26 with excised marker module: $\mathrm{Phleo}^{\mathrm{s}}, \mathrm{MSX}^{\mathrm{s}}$ \\
\hline AfS55 & reconstituted AfS01 expressing gfp-tagged $c p c A$ allele: Phleo $^{\mathrm{s}}, \mathrm{MSX}^{\mathrm{r}}$ \\
\hline Plasmids & \\
\hline pBluescript II KS & general cloning plasmid [bla, multiple cloning site] \\
\hline
\end{tabular}

Table 3.1 continued.

\begin{tabular}{|l|l|}
\hline Name & Description or sequence \\
\hline pGEM5(+) & general cloning plasmid [bla, multiple cloning site]
\end{tabular}


Chapter 3

\begin{tabular}{|c|c|}
\hline pPTRII & autonomously replicating Aspergillus plasmid $[p t r A, A M A 1, b l a]$ \\
\hline pKOBEG & pSC101 derivative expressing 1 phage redgba operon from pBAD promoter \\
\hline pMCB17 & GFP gene (pyr-4:: $\left.{ }^{p} a l c A:: g f p 2-5\right)$ in pUC19 \\
\hline pME2563 & $c p c A$ allele carrying silently mutated SalI site in coding sequence \\
\hline pME2891 & loxP-phleo ${ }^{r} / t k$ blaster $\left[\right.$ loxP- ${ }^{p}$ gpdA::ble/HSV1 tk::trpC $C^{t}$-loxP] \\
\hline pME2892 & Cre expression module in pPTRII [A. nid. niaD::cre, ptrA, AMAl] \\
\hline pME3286 & genomic $c p c C$ locus as $6.1 \mathrm{~kb}$ DraI/NaeI fragment in pGEM5(+) EcoRV \\
\hline pME3287 & template for construction of $c p c C$ deletion cassette by recombinatorial cloning \\
\hline pME3288 & cpcC::loxP-phleo ${ }^{r} / t k$-loxP replacement cassette for complete deletion \\
\hline pME3289 & construct for $c p c A D$ reconstitution by $g f p:: c p c A$ allele \\
\hline \multicolumn{2}{|c|}{ Oligonucleotides } \\
\hline Sv117 & 5'-AGA CCT ACT GAA TCT GGG TTC AGA CTT TCT ACA CCG CCC ACC GCC CCG CTC AGC TGA \\
\hline Sv118 & 5'-ACT TCA ATA CAA ATA GAA CAT AAC ATG GTA CTT CGT CAC TCG TTG ATC CTG CAT AGG \\
\hline Sv319 & 5'-ATC TTT GTC ACT CGC GTC TCA CG-3' \\
\hline Sv320 & 5'-TCT GCA CGC TGA TTG GCA GCA GC-3' \\
\hline Sv321 & 5'-TGA TTT ATG AAC TCG CCG TGT CG-3' \\
\hline Sv322 & 5'-ACG CTC CCC ACG ATG TCC AGG-3' \\
\hline Sv323 & 5'-ATT GGT TTC AAG ATA TCT AGG-3' \\
\hline Sv324 & 5'-ATC AAA GTC TTG GTT GTA TCG-3' \\
\hline Sv325 & 5'-ACA CAC CTT TGA TTG CTC GCA C-3' \\
\hline Sv326 & 5'-TCT GGT TTC AGA TCG CGG TGG-3' \\
\hline Sv327 & 5'-TGA TGC AAG CCT ATC TCA AGG-3' \\
\hline Sv328 & 5'-ACT GCC TTT TCC GAG TAG TGG-3' \\
\hline Sv329 & 5'-TTG GAA CTA CTT ACT ATG TCG -3' \\
\hline Sv330 & 5'-TTC TGG GCT CGC TGC CAT GG-3' \\
\hline Sv331 & 5'-TCA CTA GAG AAA ACA TTC GC-3' \\
\hline Sv332 & 5'-ATG TGT TTG AGA GCA GCT CGC-3' \\
\hline Sv333 & 5'-AAT GTG TTT TTG ACA CTA AGC-3' \\
\hline Sv334 & 5'-TTC TGT TCG TCT TCT TGC TCC-3' \\
\hline Sv335 & 5'-ACC TAC GGC AGT CAA GAT TGC-3' \\
\hline Sv336 & 5'-TAC TTC GTC ACT CGT TGA TCC-3' \\
\hline CS41 & 5'- TAT AGT CGA CCA GTA AAG GAG AAG AAC TT -3' \\
\hline $\mathrm{CS} 42$ & 5'- TAT ACT CGA GTA TTT GTA TAG TTC ATC CAT -3' \\
\hline
\end{tabular}




\subsection{Results}

\subsubsection{Phagocytosis by macrophages does not trigger CpcA expression in Aspergillus fumigatus}

To gain information on any requirement of balanced amino acid homeostasis in pathogenicity of Aspergillus fumigatus, we were interested whether phagocytosis of conidia by immune effector cells would elicit a Cross-Pathway Control response. For that purpose, a suitable reporter strain, AfS55, was constructed that expresses a functional $g f p:: c p c A$ allele from the native gene locus. Induction of the CPC signal transduction cascade ultimately results in increased levels of this transcriptional activator, and in in vitro control experiments a clear nuclear fluorescence was evident in this strain when starved for amino acids (fig. 3.1A). When confronted with cells of the $\mathbf{J 7 7 4}$ macrophage cell line, freshly harvested conidia from strain AfS55 were readily ingested and lysed in the phagolysosomal compartment. However, no lucid CpcA expression could be detected within these spores during the intracellular killing (fig. 3.1B). However, when phagocytosis was monitored in the presence of amino acid starvation, as induced by the histidine analogue 3-amino-1,2,4-triazole (3AT), nuclear fluorescence was evident, thus demonstrating validity of the assay. Moreover, when murine alveolar macrophages were used in the confrontation experiment, no fluorescence and therefore no expression of the CPC transcriptional activator could be monitored.

To support this observation, immunocompetent mice were infected with conidia from AfS55, and fluids from bronchoalveolar lavages were rescued after four hours containing a mixture of alveolar macrophages and ingested as well as free conidia. These samples were subjected to microscopy to show no fluorescence in the samples from infection experiments with untreated AfS55 conidia (fig. 3.1C). Also, no fluorescence could be detected throughout when spores from the wild-type isolate D141 were used, but samples from control infections with conidia from a strain expressing the GFP tag constitutively displayed a clear fluorescent signal (not shown). Accordingly, this demonstrates that the environment encountered by A. fumigatus during pulmonary infection does not trigger the Cross-Pathway Control response. 
A)

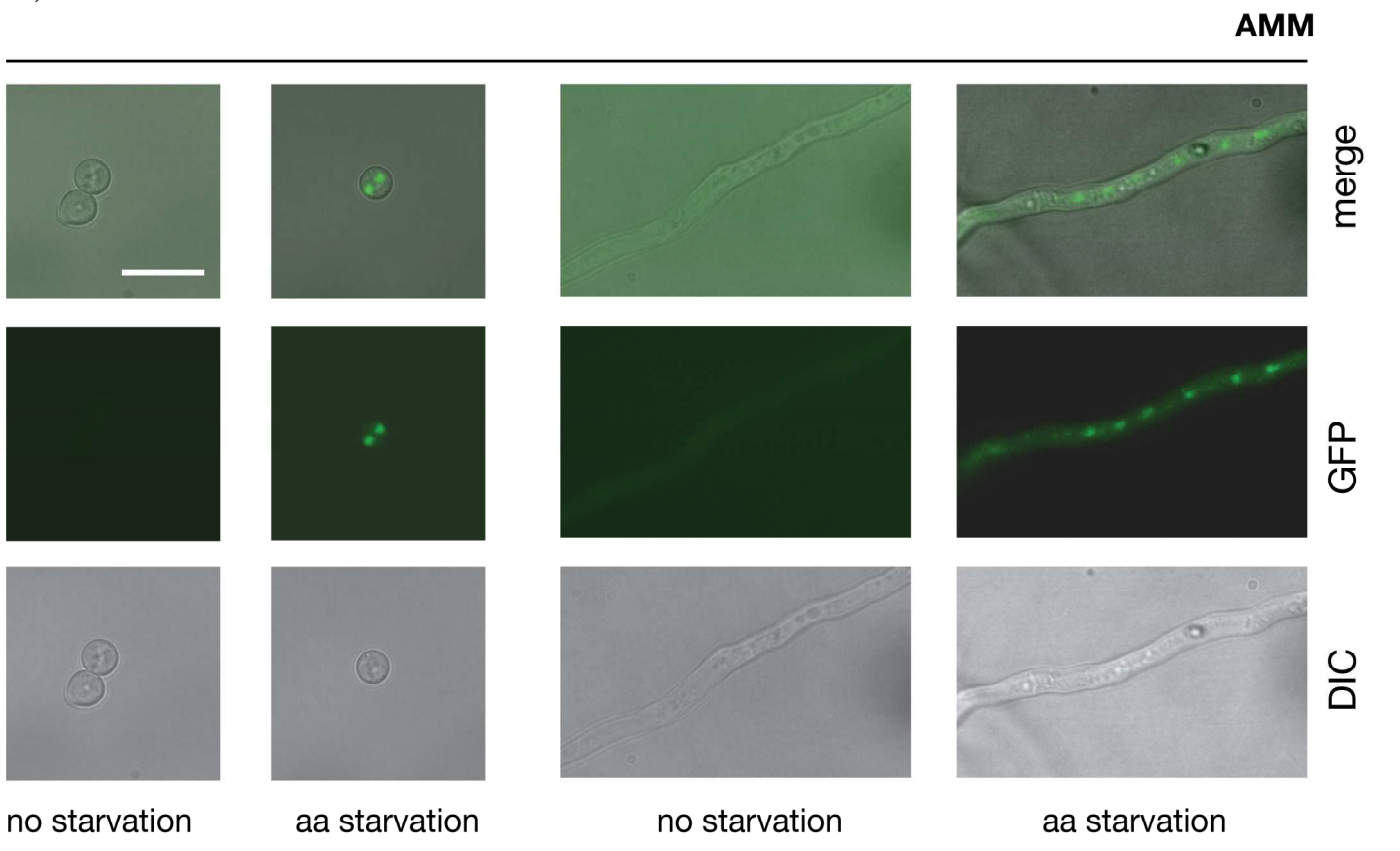

B)

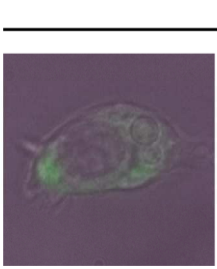

J774

AM

C)

BAL
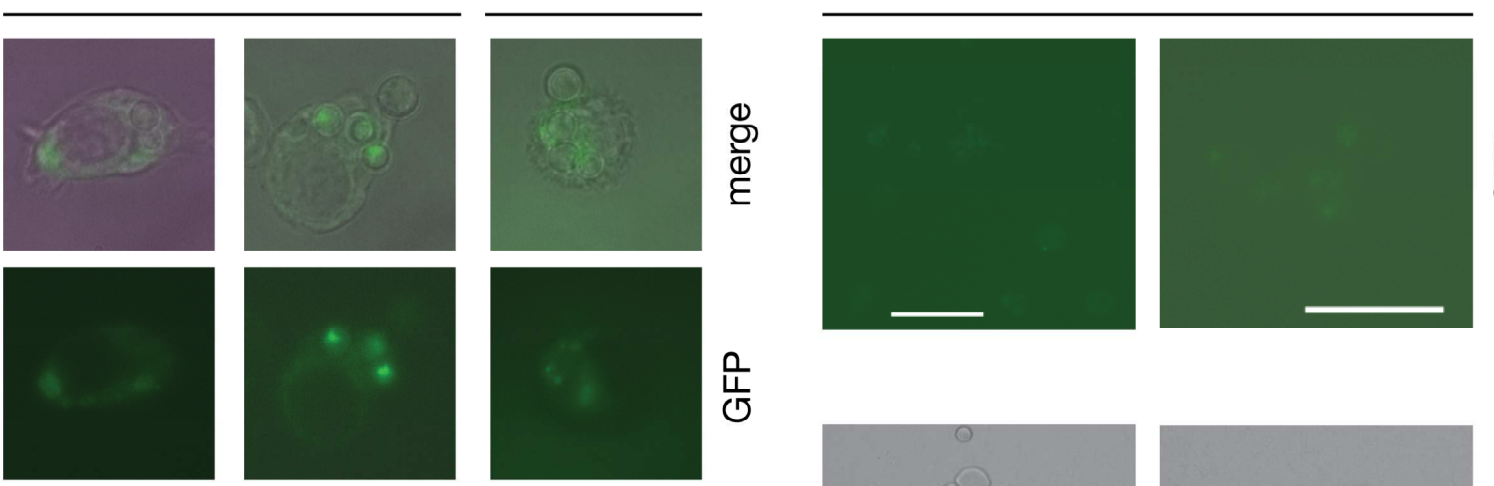

$\frac{0}{\frac{1}{1}}$

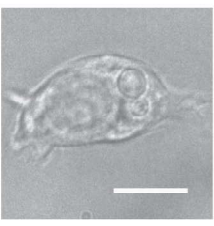

no starvation

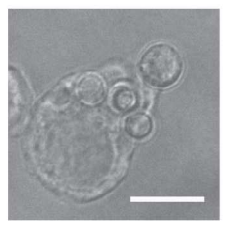

aa starvation

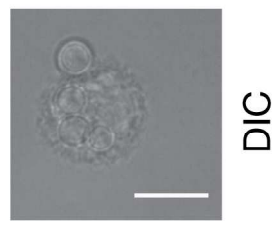

no starvation
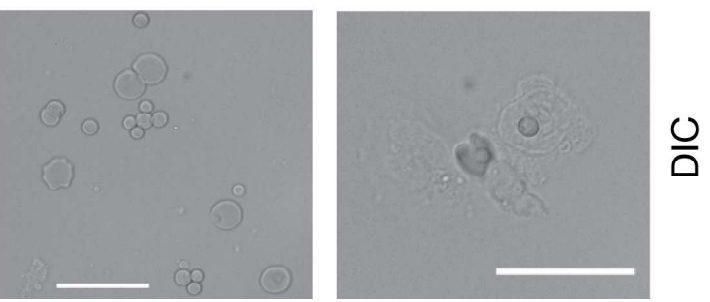

Figure 3.1. The Cross-Pathway Control response in A. fumigatus conidia is not de-repressed during confrontation with macrophages or infection of immunocompetent mice

Shown are representative images of the A. fumigatus Cross-Pathway Control reporter strain AfS55 [gfp::cpcA] propagated in Aspergillus minimal medium (AMM, A) or in cell culture medium and challenged with phagocytic cells, such as the macrophage cell line J774 or alveolar macrophages (AM, B), or when rescued after bronchoalveolar lavage (BAL, C). Starvation for the amino acid histidine to de-repress the CPC system was induced by adding the false feedback inhibitor 3-aminotriazole. Only when starved for histidine, clear nuclear fluorescence of AfS55 is evident, whereas phagocytosis ex vivo or in vivo does not elicit expression of the GFP::CpcA reporter construct. DIC: difference interference contrast; scale bars represent $10 \mu \mathrm{m}$. 


\subsubsection{The Aspergillus fumigatus genome encodes a Gcn2p-like eIF2 $\alpha$ kinase}

Eukaryotic eIF $2 \alpha$ kinases are characterised by their modular structure and contain conserved sensory, regulatory, and structural domains that flank a serine/threonine protein kinase function. In order to identify a Cross-Pathway Control sensor kinase of A. fumigatus, the genome sequence of the isolate Af293 was screened with orthologous sequences from other fungi, with the $S$. cerevisiae Gcn2p sequence serving as a structural prototype. BLAST searches revealed an annotated gene locus (Afu5g06750) with a high degree of conservation and significant similarity with respect to known fungal GC/CPC sensor kinases (fig. 3.2). Besides the characteristic catalytic domain found in such kinases, several subdomains are present in the deduced gene product of this locus: a histidyl-tRNA synthetase-related region, which is located C-terminal to the eIF2 $\alpha$ kinase domain, that mediates tRNA binding, a region resembling a degenerated kinase domain, and a $\mathrm{C}$-terminal region required for ribosome association and dimerisation (see below). The identified A. fumigatus gene encoded by this locus was designated $c p c C$, which is in agreement with the nomenclature of CPC genes identified in the filamentous ascomycetes A. nidulans and N. crassa (Sattlegger et al., 1998; Wanke et al., 1997). To confirm its genomic architecture as predicted by the automatic annotation procedure, the complete genomic $c p c C$ locus was isolated from a suitable genomic sub-library and cloned in plasmid pME3286, and in parallel the sequence of the coding region was determined from its transcript by RT-PCR. The CpcC-encoding sequence is composed of seven exons interrupted by six intronic stretches between 47 and 63 nucleotides (nt) in length. The complete coding sequence spans 4779 basepairs (bp) that correspond to 1593 codons. Accordingly, the deduced gene product has a calculated molecular weight of almost $180 \mathrm{kDa}$.

High similarities between domains of $\mathrm{CpcC}$ and that of other fungal GCN2 kinases are evident, making the classification of functional elements in the deduced primary sequence possible: Residues 41 to 154 constitute an RWD domain, which is likely to bind the GCN1/GCN20 complex. The region from position 329 to 541 resembles a kinase domain; however, due to the lack of specific invariant residues required for catalytic activity, this domain is likely to be that of a degenerate, inactive kinase analogous to the S. cerevisiae and $N$. crassa GCN2 $\psi \mathrm{PK}$ domains. Deduced from the sequence alignments and rpsblast searches, the catalytic protein kinase domain of $\mathrm{CpcC}$ resides between amino acids 580 and 937. As it is typical for eIF2 $\alpha$ kinases, two pairs of subdomains (IV-V and IX-X) in the kinase domain are separated by inserts that are variable in length and sequence. Adjacent to the eIF2 $\alpha$ kinase domain, the conserved HisRS-like domain (pos. 947 to 1443) can be found that resembles a histidyl-tRNA synthetase and binds together with the C-terminal portion the 
actual effector molecules, uncharged tRNAs. Moreover, the far C-terminus is required for dimerisation and proper localisation to the ribosome and therefore well conserved among the fungal GCN2-like kinases.

Figure 3.2. Architecture of the $c p c C$ gene from A. fumigatus

Schematic illustration of the A. fumigatus $с р с C$ gene locus with seven exons (blue boxes) interrupted by six introns; the coding region spans $5.1 \mathrm{~kb}$ on the chromosome. The deduced gene product is schematically shown underneath: $\mathrm{CpcC}$ is a protein of 1579 amino acids and contains several well-conserved elements, such as an RWD domain, a pseudo-kinase domain ( $\psi \mathrm{PK})$, the actual eIF2 $\alpha$ kinase domain (Harding et al.) and the sensor domain resembling a histidinyl-tRNA synthetase (HisRS) adjacent to it, and the C-terminal part required for dimerisaton (DD) as well as ribosome association (RB). Positions of respective domains are given as they were deduced from multiple alignments. The degree of conservation of the eIF2 $\alpha$ protein kinase domain (left) and the HisRS sensor domain (right) that binds uncharged tRNA molecules are shown by multiple primary sequence alignments including fungal CpcC counterparts from Neurospora crassa (N. c. CPC-3) and Saccharomyces cerevisiae (S. c. Gcn2p) with conserved residues shaded in black, values indicate identity percentages in pairwise alignments among the sub-domains.

\subsubsection{Deletion of $\operatorname{cpcC}$ in $A$. fumigatus impairs the Cross-Pathway Control response}

To gain information on the cellular function of the $c p c C$-encoded gene product, a procedure to create a deletion mutant was followed. Thus, a suitable deletion cassette for gene replacement by homologous recombination was generated and transformed into the clinical isolate D141, which serves as wild-type reference strain (fig. 3.3A). Several descendants were sub-cultured from the pool of primary transformants that could be isolated on corresponding selective 
media, and comprehensive Southern analyses confirmed the desired $\triangle c p c C$ genotype for several of them, from which one representative (AfS26) was chosen for further processing. The strain was transformed with an autonomously replicating plasmid (pME2892) to transiently express the Cre recombinase leading to excision of the resistance marker module (Krappmannn et al., 2005). The resulting strain AfS27 was also confirmed for its $c p c C: \because$ loxP genotype via Southern blot hybridisation to become included in the following analyses (fig. $3.3 \mathrm{~B})$.

A)

B)

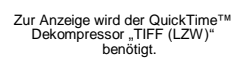

Figure 3.3. Generation of A. fumigatus $c p c C \Delta$ strains

Deletion strains of the A. fumigatus wild-type isolate D141 were generated by gene replacement with a recyclable resistance cassette. A) Outline of the wild-type $c p c C$ gene locus in strain D141 and after replacementas present in strain AfS26; additionally, the cpcC::loxP locus of descendant AfS27 resulting from marker excision by transient Cre expression is shown. The black bar indicates the region covered by the probe used in Southern analyses. B) Autoradiography from Southern blot analyses of strains A. fumigatus D141, AfS26, and AfS27. The indicated restriction enzymes were used to digest genomic DNA samples and calculated fragment sizes are given underneath; fragment positions from marker (M) lanes are shown on the right hand side.

In a first test the growth behaviour of the $\triangle c p c C$ deletion mutant was evaluated in the presence of amino acid starvation conditions. For this purpose, strains were inoculated on minimal medium containing the drug methionine sulfoximine (MSX), a glutamine synthetase inhibitor. As reference strains the wild-type progenitor D141 as well as a strain lacking the CPC transcriptional activator CpcA (AfS01) were also inoculated (fig. 3.4A). Clear differences in hyphal extension and sporulation were evident when amino acid homeostasis 
was perturbed: whereas the wild-type strain displayed proper growth, no growth could be monitored for the $\triangle c p c A$ deletion strain. Interestingly, the strain ablated for the eIF2 $\alpha$ kinase $\mathrm{CpcC}$ exhibited retarded but detectable growth on the amino acid starvation medium. Given the fact that D141 and AfS01 express different levels of the transcription factor CpcA in response to CPC derepression - zero in AfS01 $[\Delta c p c A]$ and high level expression in D141 [wild-type] - this observation is in good agreement with the proposed model of CpcA expression (see Discussion).

To evaluate the CPC response of the $\triangle c p c C$ strain in more detail, steady-state levels of reporter transcripts were determined in Northern blot hybridisations (fig. 3.4B). For that purpose, pre-grown mycelia were split and transferred into fresh minimal medium with or without a false feedback inhibitor of histidine biosynthesis (3-amino-1,2,4-triazole, 3AT) to starve strains for this amino acid. Levels of the chorismate mutase-encoding aroC transcript served as internal standard of constitutive expression, as this is an amino acid biosynthetic gene described not to be subject of CPC regulation (Krappmann et al., 1999). In contrast, $\arg B$ transcript levels increased significantly upon the onset of histidine starvation in the wildtype isolate, and this representative read-out was absent in the $\triangle c p c C$ deletion mutant strain. In further hybridisations, transcripts of the genes $c p c A$ and $c p c C$ were probed to gain additional information on the transcriptional CPC response of the mutant strain. Upon amino acid starvation, transcription levels of the key effector CpcA are strongly increased, which is probably based to a certain extent on positive feed-back regulation; in line with this assumption is the observation that in the mutant strain lacking the CPC sensor kinase this upregulation of $c p c A$ transcription was reproducibly less pronounced although still existent. This is reminiscent to the situation in the ascomycete Neurospora crassa where cpc- 1 transcript levels could be induced by amino acid starvation in a mutant deleted for the orthologous sensor kinase gene $c p c-3$ (Sattlegger et al., 1998). Moreover, a slight increase in the transcript levels upon 3AT exposure could also be detected for the $c p c C$ gene, indicating that this regulatory gene is part of the $A$. fumigatus $\mathrm{CPC}$ transcriptome.

The observed growth phenotype of a $c p c C$ null mutant with respect to amino acid starvation together with the data from Northern analyses clearly corroborate that we had identified the $\mathrm{CPC}$ sensor kinase and that it is the sole eIF2 $\alpha$ kinase required for the Cross-Pathway Control response in vitro. 
A)

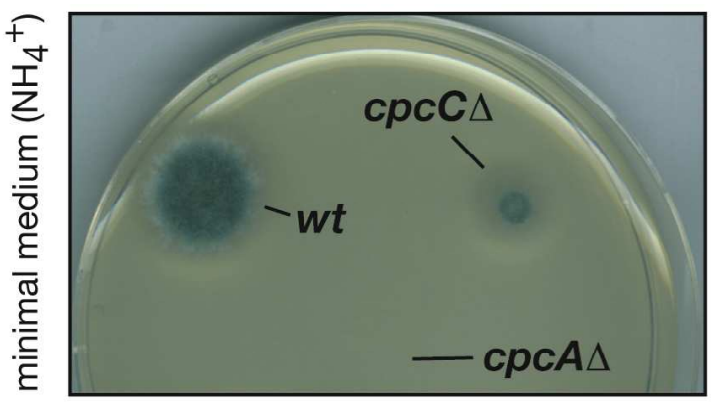

aa stavation (+ MSX)

B)

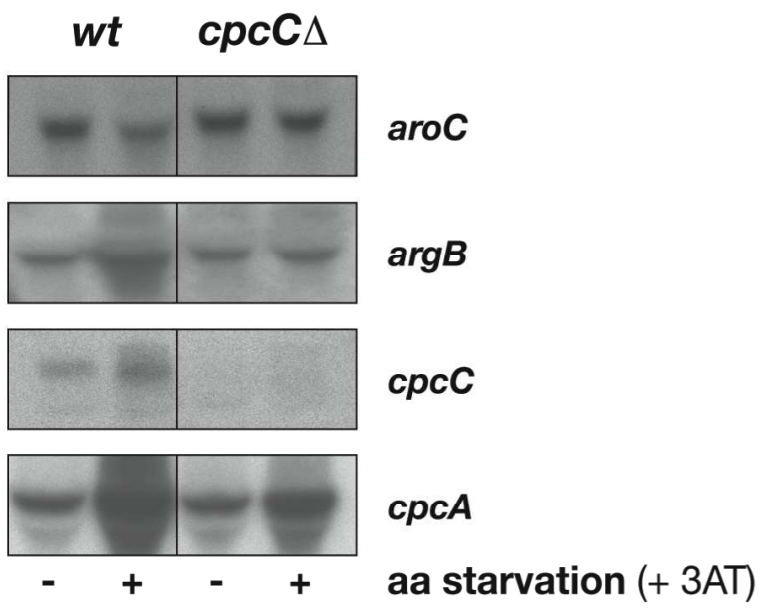

Figure 3.4. Phenotypes of a $\Delta c p c C$ mutant strain under amino acid starvation conditions

Strain AfS26 carrying the resistance marker was used for phenotypic characterisation, which behaved identical to strain AfS27 [cpcC::loxP]. A) Growth behaviour on minimal medium in the presence of glutamine starvation induced by MSX. For comparison, the clinical isolate D141 [wt] as well as the deletion strain AfS01 [ $\Delta c p c A]$ ablated for the CPC transcriptional activator is shown. B) Assessment of the CPC status by Northern analyses. Steady-state transcript levels of the constitutive internal control $\operatorname{aro} C$ and the CPC target gene $\arg B$ under sated or histidine starvation conditions (+3AT) clearly demonstrate the $\mathrm{CPC}^{-}$phenotype of the $\triangle c p c C$ strain. Moreover, hybridisation signals from corresponding probes indicate an increase in $c p c C$ transcription under amino acid starvation conditions, and that transcriptional induction of $c p c A$ expression depends partially on the presence of the $c p c C$-encoded eIF2 $\alpha$ kinase.

\subsubsection{A cpcC null mutant of $A$. fumigatus is still able to phosphorylate eIF2 $\alpha$}

As the $c p c C$ gene is assumed to encode a kinase that acts on the translation initiation factor eIF2, the degree of phosphorylation was followed upon derepression of the CPC system. For this purpose, the cross-reaction of specific antibodies raised against the $\alpha$-subunit of eIF2 was monitored in Western experiments, and a biochemical assay was employed to validate the CPC status. As expected, phosphorylation of eIF2 $\alpha$ increased from a basal level when an A. fumigatus wild-type strain was shifted to amino acid starvation conditions using various 
inhibitors of fungal amino acid biosynthesis (fig. 3.5A). In accordance with this result, enzymatic activities of the $\arg B$-encoded OTCase were elevated (fig. 3.5B). To our surprise, the elevated eIF2 $\alpha-\mathrm{P}$ signal was also clearly and reproducibly detectable from crude extracts that had been prepared from the $\triangle c p c C$ deletion mutant strain cultivated under identical conditions. OTCase activities determined from these crude extracts, however, revealed no increase and hence confirmed the $\mathrm{cpc}^{-}$phenotype of the $\Delta c p c C$ mutant background. Given the high specificity of the phospho-eIF $2 \alpha$ antibody, these data suggest that $\mathrm{CpcC}$ is not the only eIF2 $\alpha$ kinase encoded in the A. fumigatus genome, however its action is specific and strictly required for a proper Cross-Pathway Control response of this fungus to counteract amino acid deprivation.

A)

B)

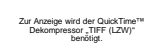

Figure 3.5. eIF2 $\alpha$ phosphorylation is not abolished in a $\triangle c p c C$ background

A) Western experiments using crude extracts from strains D141 [wild-type] and AfS26 [ $\triangle c p c C]$ starved for tryptophan (W) by 5-methyltryptophan (5MT) or histidine $(\mathrm{H})$ by $3 \mathrm{AT}$ are shown, in which polyclonal antibodies raised against eIF2 $\alpha$ were used as internal standard and a specific one to monitor the amount of eIF2 $\alpha$ phosphorylated on Ser51. B) Parallel determination of $\arg B$-encoded OTCase activities support the $\mathrm{cpc}^{-}$phenotype of the $\triangle c p c C$ strain to indicate that $\mathrm{CpcC}$-specific phosphorylation of eIF $2 \alpha$ is required for a proper Cross-Pathway Control response.

\subsubsection{Virulence of $A$. fumigatus is not affected in a $\triangle c p c C$ background}

In a previous study we had demonstrated that the transcriptional activator of the A. fumigatus CPC system is required for full virulence in a murine model of pulmonary aspergillosis (Krappmann et al., 2004): mutant strains deleted for the encoding cpcA gene appeared attenuated in infection studies but did not show any obvious phenotype in vitro except sensitivity towards amino acid analogues. To elucidate whether a derepressed CPC system is necessary for full virulence, both $\Delta c p c C$ strains AfS26 [cpcC::loxP-phleo $\left.{ }^{R} / t k\right]$ and AfS27 $[$ сpсC::loxP] were used to infect cohorts of leukopenic mice that had been immunocompromised following a standard protocol using hydrocortisone and 
cyclophosphamide. Health conditions of the animals were monitored over a time period of up to ten days to assess weight loss or severe signs of pulmonary distress. As reference, again the clinical isolate and progenitor strain D141 was used, which resulted in killing of twelve mice of a 13-animal cohort in the experimental time frame (fig. 3.6). Both mutant strains, however, also caused the onset of pulmonary aspergillosis in all infected animals to result in almost exceptionless killing of the experimental groups. Median survival times were in the same range for all three strains tested in this experimental series, so no differences with respect to virulence were evident between the wild-type and the $\Delta c p c C$ mutant background.

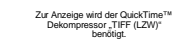

Figure 3.6. Virulence of $A$. fumigatus $\Delta c p c C$ strains is unaltered in a murine model of pulmonary aspergillosis. Survival plots for groups of leukopenic CD1 mice intranasally infected with $2 \times 10^{5}$ conidia of A. fumigatus strains D141 [wild-type] (left panel) or $\Delta c p c C$ deletion mutants (right panel) are shown. Animals received an intraperitoneal dose of $150 \mathrm{mg} / \mathrm{kg}$ cyclophosphamide on days $-3,-1,2$, and every third day plus a subcutaneous dose of $112.5 \mathrm{mg} / \mathrm{kg}$ hydrocortisone acetate on day -1 . No virulence differences for this set of strains is could be deduced in this animal model for pulmonary aspergillosis. 


\subsection{Discussion}

Invasive diseases caused by aspergilli are characterised by the impact of numerous factors that influence the outcome of the fungus/host interaction. It is generally assumed that the natural habitat selects for traits that contribute to the pathogenicity of an opportunistic pathogen. Accordingly, common cellular and physiological attributes represent virulence-determining factors and add to the fungal virulome. Among the most important fungal determinants, nutritional versatility as well as stress resistance have to be considered, as both qualities influence the in vivo growth rate, which is directly correlated to virulence (Rhodes, 2006).

The environment encountered by A. fumigatus upon infection represents a specific ecological niche that is possibly stressful, especially with respect to nutritional supply, so the pathogenic potential of this particular Aspergillus species implies that A. fumigatus is well equipped and adapted to utilize the surrounding tissue. To gain insight into the mechanism of aspergillosis, comprehensive knowledge of factors that support infection and in vivo growth is required, and therefore metabolic routes that support fungal survival in this possibly hostile environment are of interest. Besides components of primary routes, regulatory cascades that act on clusters of metabolic pathways are informative targets in phenotypic mutant analyses, and one prominent signal transduction pathway that relates environmental stress to fungal physiology is represented by eIF2 $\alpha$ kinase signalling. Previous studies have demonstrated that the terminal effector of this cascade, the $\mathrm{CpcA}$ transcriptional activator, is required for full virulence of $A$. fumigatus in a murine model of pulmonary aspergillosis (Krappmann et al., 2004). Increased expression of CpcA, however, does not occur upon ingestion by macrophages, which act as primary defence line when spores of this fungal pathogen are inhaled down to the alveoli (Ibrahim-Granet et al., 2003). Accordingly, phagocytosis by macrophages appears not to induce the Cross-Pathway Control system in A. fumigatus conidia. This implies that the microenvironment of the macrophage phagosome contains sufficient amounts of amino acids and represents a balanced environment with respect to amino acid homeostasis. This observation is in line with previous studies monitoring the immediate transcriptional reprogramming of Candida albicans cells after phagocytosis by immune effector cells: ingestion by neutrophils but not macrophages results in an amino acid starvation response by inducing biosynthetic genes of the arginine pathway in a Gen4p-dependent manner (RubinBejerano et al., 2003). The specific transcriptional response of $C$. albicans after ingestion by macrophages is characterised by induction of alternative carbon metabolism, enhanced nutrient acquisition, and repression of the translational machinery, but not de-repression of the General Control system (Lorenz et al., 2004). This shift of C. albicans confronted with 
macrophages to a starvation mode that is distinct from the conventional GC/CPC response was recently substantiated by proteome studies (Fernandez-Arenas et al., 2007). In view of that and our macrophage ingestion data with an A. fumigatus $\mathrm{CpcA}$ reporter strain, an operative Cross-Pathway Control appears obsolete for a fungal pathogen in the course of phagocytosis by macrophages.

In this study we were able to reveal that the upstream signalling sensor, the eIF $2 \alpha$ kinase $\mathrm{CpcC}$, appears to be redundant for pathogenicity of $A$. fumigatus, as indicated by unaffected virulence capacities of corresponding deletion mutant strains in an infection model using leukopenic mice. This kind of model for pulmonary aspergillosis was chosen for several reasons: First, the preceding studies on the $\Delta c p c A$ mutant had been carried out in the same model, making virulence characteristics of $A$. fumigatus $\triangle c p c A$ and $\triangle c p c C$ mutants comparable. Second, we were interested in virulence based on growth characteristics and the ability to exploit the infected tissue as substrate. In contrast to this, infection models based on hydrocortisone treatment solely do not result in depletion of neutrophils, and there, more subtle effects resulting from the interaction of A. fumigatus strains with the host's innate immune system may be gained, which is out of the scope of this particular study. Given the complex mechanism of $\mathrm{CpcA}$ expression, the negative result on full virulence of $\Delta c p c C$ deletants indicates that basal but not elevated levels of this transcription factor are sufficient but also necessary to support virulence of A. fumigatus. Transcription from the cpcA locus results in mRNA molecules from which $\mathrm{CpcA}$ is translated at low levels due to the leakiness of translational barriers in the 5' leader region. The onset of starvation, which is sensed by the $\mathrm{CpcC}$ kinase, relieves the translational block mediated by these upstream open reading frames (uORFs) to result in high levels of CpcA. Accordingly, when the function of the sensor kinase is impaired, no de-repression of the CPC system can occur; however, basal levels of CpcA are steadily expressed due to enduring transcription of the encoding gene. The existence of such basal but un-inducible levels of the transcriptional activator are clearly mirrored by the attenuated, intermediate $\mathrm{MSX}^{\mathrm{S}}$ growth phenotype of a $\Delta c p c C$ mutant. The assumption that the CPC system is not de-repressed during aspergillosis is substantiated by additional studies: first, monitoring in vivo levels of $c p c A$ transcripts by competitive RT-PCR revealed constant levels of gene expression (Zhang et al., 2005), and second, preliminary in vivo transcriptome profiles are clearly distinct from the data set that is generated in response to amino acid starvation (our unpublished results). Conclusively, no impact of the CPC signal transduction pathway on A. fumigatus pathogenesis can be deduced, arguing for the absence of nutritional stress conditions with respect to amino acid homeostasis in the murine lung. However, our 
data do not exclude the presence of a redundant signalling pathway that might function through an alternative sensor kinase. As indicated in our Western experiments monitoring the eIF2 $\alpha$ phosphorylation status, additional eIF2 $\alpha$ kinase activities are present in A. fumigatus. However, action of $\mathrm{CpcC}$ is specific for and strictly required for a proper Cross-Pathway Control response of this fungus to counteract amino acid deprivation. Inspection of the A. fumigatus genome sequence indeed confirms the existence of a second eIF2 $\alpha$ kinase, the ifkB (for initiation factor kinase B) gene product. Our preliminary characterisation of this gene and its gene product implies that this kinase is functionally not redundant to $\mathrm{CpcC}$ but is, however, responsible for the residual eIF2 $\alpha$ phosphorylation in a $\Delta c p c C$ background: an $\triangle i f k B$ mutant strain is not impaired in its $\mathrm{CPC}$ response and an $\Delta i f k B ; \Delta c p c C$ double deletion mutant does not display phosphorylation of eIF2 $\alpha$ in Western experiments (data not shown). Moreover, no clear cellular role could be assigned to the IfkB kinase through our preliminary phenotypic studies, and we consider it unlikely that this eIF2 $\alpha$ kinase affects virulence of A. fumigatus in our murine model of pulmonary aspergillosis.

Assuming that uninduced $\mathrm{CpcA}$ levels contribute to virulence of A. fumigatus raises the question, which sub-set of genes is targeted and driven in their expression by low CpcA quantities. The existence of such genes could be demonstrated in S. cerevisiae (Paravicini et $a l ., 1989)$ and it is likely that in A. fumigatus several genes of that kind exist. Comprehensive profiling data on the $\mathrm{CpcA}$-dependent but $\mathrm{CPC}$-uninduced transcriptome and proteome will assist in defining such a basal targetome of this conserved transcription factor to gain further knowledge on the influence of the Cross-pathway Control system in pathogenicity of A. fumigatus. 


\subsection{References}

Aufauvre-Brown, A., Brown, J.S., and Holden, D.W. (1998) Comparison of virulence between clinical and environmental isolates of Aspergillus fumigatus. Eur J Clin Microbiol Infect Dis 17: 778-780.

Behnsen, J., Narang, P., Hasenberg, M., Gunzer, F., Bilitewski, U., Klippel, N., Rohde, M., Brock, M., Brakhage, A.A., and Gunzer, M. (2007) Environmental dimensionality controls the interaction of phagocytes with the pathogenic fungi Aspergillus fumigatus and Candida albicans. PLoS Pathog 3: e13.

Bradford, M.M. (1976) A rapid and sensitive method for the quantitation of microgram quantities of protein utilizing the principle of protein-dye binding. Anal Biochem 72: 248-254.

Brakhage, A.A. (2005) Systemic fungal infections caused by Aspergillus species: epidemiology, infection process and virulence determinants. Curr. Drug. Targets 6: 875-886.

Brown, T., and Mackey, K. (1997) Analysis of RNA by Northern and Slot Blot Hybridization. In Current Protocols in Molecular Biology: John Wiley \& Sins, Inc., pp. 4.9.1-4.9.16.

Carsiotis, M., and Jones, R.F. (1974) Cross-pathway regulation: tryptophan-mediated control of histidine and arginine biosynthetic enzymes in Neurospora crassa. J Bacteriol 119: 889-892.

Carsiotis, M., Jones, R.F., and Wesseling, A.C. (1974) Cross-pathway regulation: histidinemediated control of histidine, tryptophan, and arginine biosynthetic enzymes in Neurospora crassa. J Bacteriol 119: 893-898.

Casadevall, A., and Pirofski, L.A. (2003) The damage-response framework of microbial pathogenesis. Nat. Rev. Microbiol. 1: 17-24.

Casadevall, A., Steenbergen, J.N., and Nosanchuk, J.D. (2003) 'Ready made' virulence and 'dual use' virulence factors in pathogenic environmental fungi - the Cryptococcus neoformans paradigm. Curr. Opin. Microbiol. 6: 332-337.

Casadevall, A. (2006) Cards of virulence and the global virulome for humans. Microbe 1: 359-364.

Chaveroche, M.K., Ghigo, J.M., and d'Enfert, C. (2000) A rapid method for efficient gene replacement in the filamentous fungus Aspergillus nidulans. Nucleic Acids Res 28 : E97.

Dower, W.J., Miller, J.F., and Ragsdale, C.W. (1988) High efficiency transformation of $E$. coli by high voltage electroporation. Nucleic Acids Res 16: 6127-6145.

Feinberg, A.P., and Vogelstein, B. (1983) A technique for radiolabeling DNA restriction endonuclease fragments to high specific activity. Anal Biochem 132: 6-13.

Fernandez-Abalos, J.M., Fox, H., Pitt, C., Wells, B., and Doonan, J.H. (1998) Plant-adapted green fluorescent protein is a versatile vital reporter for gene expression, protein localization and mitosis in the filamentous fungus, Aspergillus nidulans. Mol Microbiol 27: 121-130.

Fernandez-Arenas, E., Cabezon, V., Bermejo, C., Arroyo, J., Nombela, C., Diez-Orejas, R., and Gil, C. (2007) Integrated proteomics and genomics strategies bring new insight into Candida albicans response upon macrophage interaction. Mol Cell Proteomics 6: 460-478.

Grundmann, O., Mosch, H.U., and Braus, G.H. (2001) Repression of GCN4 mRNA translation by nitrogen starvation in Saccharomyces cerevisiae. J Biol Chem 276: 25661-25671.

Hanahan, D., Jessee, J., and Bloom, F.R. (1991) Plasmid transformation of Escherichia coli and other bacteria. Methods Enzymol 204: 63-113. 
Harding, H.P., Zhang, Y., Zeng, H., Novoa, I., Lu, P.D., Calfon, M., Sadri, N., Yun, C., Popko, B., Paules, R., Stojdl, D.F., Bell, J.C., Hettmann, T., Leiden, J.M., and Ron, D. (2003) An integrated stress response regulates amino acid metabolism and resistance to oxidative stress. Mol Cell 11: 619-633.

Hoffmann, B., Valerius, O., Andermann, M., and Braus, G.H. (2001) Transcriptional autoregulation and inhibition of mRNA translation of amino acid regulator gene $c p c A$ of filamentous fungus Aspergillus nidulans. Mol Biol Cell 12: 2846-2857.

Ibrahim-Granet, O., Philippe, B., Boleti, H., Boisvieux-Ulrich, E., Grenet, D., Stern, M., and Latgé, J.P. (2003) Phagocytosis and intracellular fate of Aspergillus fumigatus conidia in alveolar macrophages. Infect Immun 71: 891-903.

Käfer, E. (1977) Meiotic and mitotic recombination in Aspergillus and its chromosomal aberrations. Adv Genet 19: 33-131.

Kolar, M., Punt, P.J., van den Hondel, C.A., and Schwab, H. (1988) Transformation of Penicillium chrysogenum using dominant selection markers and expression of an Escherichia coli lacZ fusion gene. Gene 62: 127-134.

Krappmann, S., Helmstaedt, K., Gerstberger, T., Eckert, S., Hoffmann, B., Hoppert, M., Schnappauf, G., and Braus, G.H. (1999) The aroC gene of Aspergillus nidulans codes for a monofunctional, allosterically regulated chorismate mutase. J Biol Chem 274: 22275-22282.

Krappmann, S., Bignell, E.M., Reichard, U., Rogers, T., Haynes, K., and Braus, G.H. (2004) The Aspergillus fumigatus transcriptional activator CpcA contributes significantly to the virulence of this fungal pathogen. Mol. Microbiol. 52: 785-799.

Krappmann, S., Bayram, O., and Braus, G.H. (2005) Deletion and allelic exchange of the Aspergillus fumigatus veA locus via a novel recyclable marker module. Eukaryot Cell 4: 1298-1307.

Lorenz, M.C., Bender, J.A., and Fink, G.R. (2004) Transcriptional response of Candida albicans upon internalization by macrophages. Eukaryot Cell 3: 1076-1087.

Paravicini, G., Mösch, H.-U., Schmidheini, T., and Braus, G. (1989) The general control activator protein GCN4 is essential for a basal level of $A R O 3$ gene expression in Saccharomyces cerevisiae. Mol Cell Biol 9: 144-151.

Punt, P.J., and van den Hondel, C.A. (1992) Transformation of filamentous fungi based on hygromycin B and phleomycin resistance markers. Methods Enzymol 216: 447-457.

Rhodes, J.C. (2006) Aspergillus fumigatus: Growth and virulence. Med Mycol 44 Suppl: 7781.

Romano, P.R., Garcia-Barrio, M.T., Zhang, X., Wang, Q., Taylor, D.R., Zhang, F., Herring, C., Mathews, M.B., Qin, J., and Hinnebusch, A.G. (1998) Autophosphorylation in the activation loop is required for full kinase activity in vivo of human and yeast eukaryotic initiation factor 2alpha kinases PKR and GCN2. Mol Cell Biol 18: 22822297.

Rubin-Bejerano, I., Fraser, I., Grisafi, P., and Fink, G.R. (2003) Phagocytosis by neutrophils induces an amino acid deprivation response in Saccharomyces cerevisiae and Candida albicans. Proc Natl Acad Sci U S A 100: 11007-11012.

Saiki, R.K., Bugawan, T.L., Horn, G.T., Mullis, K.B., and Erlich, H.A. (1986) Analysis of enzymatically amplified beta-globin and HLA-DQ alpha DNA with allele-specific oligonucleotide probes. Nature 324: 163-166.

Sambrook, J., Fritsch, E.F., and Maniatis, T. (1989) Molecular Cloning: A Laboratory Manual. Cold Spring Harbor, NY: Cold Spring Harbor Laboratory Press.

Sattlegger, E., Hinnebusch, A.G., and Barthelmess, I.B. (1998) cpc-3, the Neurospora crassa homologue of yeast $G C N 2$, encodes a polypeptide with juxtaposed eIF2alpha kinase and histidyl-tRNA synthetase-related domains required for general amino acid control. J Biol Chem 273: 20404-20416. 
Smith, J.M., Tang, C.M., Van Noorden, S., and Holden, D.W. (1994) Virulence of Aspergillus fumigatus double mutants lacking restriction and an alkaline protease in a low-dose model of invasive pulmonary aspergillosis. Infect Immun 62: 5247-5254.

Southern, E.M. (1975) Detection of specific sequences among DNA fragments separated by gel electrophoresis. J Mol Biol 98: 503-517.

Staib, F., Mishra, S.K., Rajendran, C., Voigt, R., Steffen, J., Neumann, K.H., Hartmann, C.A., and Heins, G. (1980) A notable Aspergillus from a mortal aspergilloma of the lung. New aspects of the epidemiology, serodiagnosis and taxonomy of Aspergillus fumigatus. Zentralbl Bakteriol A 247: 530-536.

Tang, C.M., Cohen, J., Krausz, T., Van Noorden, S., and Holden, D.W. (1993) The alkaline protease of Aspergillus fumigatus is not a virulence determinant in two murine models of invasive pulmonary aspergillosis. Infect Immun 61: 1650-1656.

Tian, G., Lim, D., Oppenheim, J.D., and Maas, W.K. (1994) Explanation for different types of regulation of arginine biosynthesis in Escherichia coli B and Escherichia coli K12 caused by a difference between their arginine repressors. J Mol Biol 235: 221-230.

Walsh, T.J., and Groll, A.H. (2001) Overview: non-fumigatus species of Aspergillus: perspectives on emerging pathogens in immunocompromised hosts. Curr. Opin. Investig. Drugs. 2: 1366-1367.

Wanke, C., Eckert, S., Albrecht, G., van Hartingsveldt, W., Punt, P.J., van den Hondel, C.A., and Braus, G.H. (1997) The Aspergillus niger GCN4 homologue, cpcA, is transcriptionally regulated and encodes an unusual leucine zipper. Mol Microbiol 23: 23-33.

Wek, R.C., Jiang, H.Y., and Anthony, T.G. (2006) Coping with stress: eIF2 kinases and translational control. Biochem. Soc. Trans. 34: 7-11.

Wek, S.A., Zhu, S., and Wek, R.C. (1995) The histidyl-tRNA synthetase-related sequence in the eIF-2 alpha protein kinase GCN2 interacts with tRNA and is required for activation in response to starvation for different amino acids. Mol Cell Biol 15: 44974506.

Woodcock, D.M., Crowther, P.J., Doherty, J., Jefferson, S., DeCruz, E., Noyer-Weidner, M., Smith, S.S., Michael, M.Z., and Graham, M.W. (1989) Quantitative evaluation of Escherichia coli host strains for tolerance to cytosine methylation in plasmid and phage recombinants. Nucleic Acids Res 17: 3469-3478.

Woolhouse, M.E.J. (2006) Where do emerging pathogens come from? Microbe 1: 511-515.

Zhang, L., Wang, M., Li, R., and Calderone, R. (2005) Expression of Aspergillus fumigatus virulence-related genes detected in vitro and in vivo with competitive RT-PCR. Mycopathologia 160: 201-206. 


\section{Chapter 4}

\section{The Basal Proteome Directed by the Aspergillus fumigatus Transcriptional Regulator CpcA}

\subsection{Abstract}

The opportunistic pathogenic fungus A. fumigatus requires for full virulence the transcriptional regulator $\mathrm{CpcA}$, as a deletion of this gene leads to reduced virulence. However, the sensor kinase $\mathrm{CpcC}$ is not required for infection indicating that the basal expression level of CpcA is sufficient to support pathogenicity. In this study a part of the basal CPCome via proteomic experiments was analysed by comparing the wild-type D141 isolate with its $\Delta c p c A$ deletion derivative under sated conditions. By DIGE analysis 22 regulated proteins with more than a 1.4 fold induction/repression could be identified, with 16 of these being increased in the wild-type and eight showing a decreased amount compared to the deletion strain. Classification via annotation made it possible to place them in different groups of function. Three proteins were identified that may be involved in pathogenicity of $A$. fumigatus, e.g. the Asp hemolysine. Furthermore proteins needed for metabolism, which form the largest group, and one protein having the function to neutralise reactive oxidative species were also found. With the aim to identify putative CpcA binding sites the promoter regions of the encoding genes were investigated. Four typical sequences known as putative recognition sites of the transcriptional factor were checked and found in eight genes of the regulated proteins. These data shed first light on the basal CPCome, which is necessary but also sufficient to support pulmonary aspergillosis. 


\subsection{Introduction}

The air borne filamentous fungus A. fumigatus has been recognised to be one of the most common organisms to evoke invasive aspergillosis (Soubani and Chandrasekar, 2002). By producing conidia with a size about $2-3 \mu \mathrm{m}$ in diameter the fungus has the ability to reach easily the lung aveoli of the host (Latgé, 1999); (Brakhage and Langfelder, 2002). Moreover, its pronounced thermotolerance with an optimal growth temperature at $37^{\circ} \mathrm{C}$ and a survival up to $55^{\circ} \mathrm{C}$ makes it possible that $A$. fumigatus can grow very well in mammalians (Chang et al., 2004; Cooney and Emerson, 1964; Maheshwari et al., 2000). Like other pathogenic organisms the fungus needs special abilities to survive in the host whereas limited nutrients and the immune defence system are the main barriers, which must be overwhelmed. Incoming spores are attacked by macrophages and neutrophils as primary defence response of the host. These phagocytosing cells induce oxidative stress by using reactive oxidant intermediates (ROI) to kill the spores (Philippe et al., 2003). Therefore, defence mechanisms as the mitogen-activated kinases (MAPKs), which constitute a regulation system, that enables the fungus the adaptation to environmental stress like neutralisation of oxidative molecules are required. Four different proteins of the MAPKs are found in A. fumigatus called SakA, MpkA, MpkB and MpkC (May et al., 2005). Other enzymes like glutathione transferases are needed for inactivation of reactive oxidative species (ROS) as well. In A. fumigatus there are three of these enzymes that show a peroxidase activity, by which the fungus has the possibility to detoxify of oxidative intermediates (Burns et al., 2005).

As mentioned above the ability of A. fumigatus to respond on the defence mechanism of the host is as important as the competency to get nutrients from the surroundings. The lung, which is the primary target of incoming spores, is a place where substrates and nutrients are complex and unbalanced. This organ consists mainly of proteins like collagen (Bromley and Donaldson, 1996; Gil et al., 1996; Penalver et al., 1996; Tronchin et al., 1993) so that there is no abundance of free nitrogen and carbon sources. To grow under these circumstances degrading proteins and pathways for starvation conditions are indispensable for the fungus. Therefore proteases are necessary for the fragmentation of lung components into peptides. Afterwards up take systems consisting of oligopeptide transporters are involved to transport the nutrients into the fungus as described in earlier works for $C$. albicans (Reuss and Morschhäuser, 2006) whereas nitrogen and carbon become available for the organism. Not only proteases but also pathways to recognise the starvation conditions in the surrounding and to activate genes involved in metabolism like amino acid biosynthesis are important. One of these pathways for recognition nutrient limitation resulting in a regulation of different kinds 
of genes in the fungus is the Cross Pathway Control (CPC), the homologous system to the General Control (GC) in S. cerevisiae (Hinnebusch, 1984; Hoffmann et al., 2001; Krappmann et al., 2004). The CPC consists of a sensor kinase called CpcC and a transcriptional regulator $\mathrm{CpcA}$. The $c p c A$ gene is regulated on transcriptional level and on translational level (Hoffmann et al., 2001; Krappmann et al., 2004). Under non-starvation conditions where nutrients are available for the fungus the $c p c A$ translational level is repressed to result in a basal expression level of CpcA. Responsible for the repression is the high amount of unphosphorylated initiation factor for translation eIF2. This leads to a translation of the two small uORFs within the promoter region of the $c p c A$ gene, which are translational regulatory elements. Under starvation conditions, uncharged tRNAs are recognised by $\mathrm{CpcC}$ leading to a phosphorylation of eIF2 to eIF2 P. As a result the coding sequence of CpcA is translated leading to an increased amount of this transcriptional activator (Braus et al., 2004). With more than 500 target genes $\mathrm{CpcA}$ belongs to the global regulators within the fungus. Transcriptomic analysis of the CPC/GC under starvation conditions was performed in different organisms like S. cerevisiae, N. crassa and A. fumigatus (Natarajan et al., 2001; Tian et al., 2007) and the comparison of these data show that there are a lot of similarities of the regulated group of genes. In all organisms amino acid biosynthesis genes are up-regulated as well as genes involved in carbon and nitrogen metabolism (Natarajan et al., 2001; Tian et al., 2007). Overall the $\mathrm{CPC} / \mathrm{GC}$ give the different organisms the ability to react on changing environmental and nutritional conditions.

Earlier studies indicated a role for $\mathrm{CpcA}$ in pathogenicity of A. fumigatus. Deletion of the transcriptional regulator decreased the virulence of this fungus in a murine mouse model in comparison to the wild-type strain (Krappmann et al., 2004). In contrast to that a $\Delta c p c C$ strain displays no reduction in virulence in immuno-compromised mice (see Chapter 2). In vitro and in vivo experiments with a $g f p:: c p c A$ construct displayed no induction of the CPC. These results lead to the suggestion, that the basal expression level of $\mathrm{CpcA}$ is sufficient for full virulence and that de-repression of $\mathrm{CpcA}$ expression is not required in invasive aspergillosis (Sasse et al., 2008).

In this study A. fumigatus genes regulated in dependency of the basal CpcA expression level were identified by a proteome approach. The proteomic profiles of a wild-type isolate and its $\triangle c p c A$ deletion strain were compared under sated conditions to reveal an incomplete section of the basal CpcA-directed proteome of A. fumigatus. 


\subsection{Materials and Methods}

\subsubsection{Media, culture conditions and strains}

The wild-type isolate D141 (Staib et al., 1980) and the $\Delta c p c A$ deletion strain AfS01 (Krappmann et al., 2004) were used in these experiments. Inoculation was made in liquid minimal medium supplemented according to Käfer (1977). As nitrogen source ammonium was used (10mM final concentration). Each strain was inoculated in $400 \mathrm{ml}$ minimal medium with $2 \times 10^{6}$ spores $/ \mathrm{ml}$ and incubated at $37^{\circ} \mathrm{C}$ for $18 \mathrm{~h}$. Mycelia were harvested by filtration through miracloth filter (CALBIOCHEM) washed twice with sterile water and subsequently ground in liquid nitrogen.

\subsubsection{Protein extraction and determination of concentration}

$50 \mathrm{mg}$ of ground mycelium was transferred to an Eppendorf reaction cup. Extraction was carried out by using the TCA/aceton precipitation method as described by Darmerval et al. (1986) with some modifications. The $50 \mathrm{mg}$ of mycelium were incubated with $500 \mu \mathrm{l}$ acetone $/ 13.3 \% \mathrm{w} / \mathrm{v}$ trichloracetacid and $0.09 \% \mathrm{w} / \mathrm{v}$ DTT over night. After centrifugation at $14000 \mathrm{rpm}$ for $15 \mathrm{~min}$ at $4^{\circ} \mathrm{C}$ the supernatant was discarded and the pellet was washed with 1 $\mathrm{ml}$ ice-cold acetone containing $0.009 \%$ w/v DTT. For removal the wash solution centrifugations were done. Afterwards the pellet was dried $5 \mathrm{~min}$ at RT. $300 \mu$ lysis buffer [7 M urea, 2 M thiourea, 2\% (w/v) CHAPS, 1\% (w/v) Zwittergent 3-10, 0.8\% (v/v) IPG buffer pH 3-11, $20 \mathrm{mM}$ DTT, $20 \mathrm{mM}$ Tris] described by Kniemeyer et al. (2006) with some modifications was added for resuspension the pellet and the received solution was incubated for $1 \mathrm{~h}$ at room temperature. The supernatant was collected after centrifugation at $14000 \mathrm{rpm}$ at $16^{\circ} \mathrm{C}$ for $30 \mathrm{~min}$. The protein concentration was determined according to the protocol of Bradford (1976). Therefore BIO Rad protein assay (BIO-RAD Lab., Hartfordshire, USA) was used.

\subsubsection{2-D gel electrophoresis}

For pre-experiments 2-D gel electrophoresis according to the protocol of Kniemeyer et al. (2006) was performed. In the experiments strips of $24 \mathrm{~cm}$ with a non-linear $\mathrm{pH}$ gradient of 311 (Healthcare Biosciences, GE) were used and rehydrated over night in $450 \mu$ l rehydration buffer [7 M urea, $2 \mathrm{M}$ thiourea, $2 \%$ (w/v) CHAPS, 1\% Zwittergent, $0.002 \%$ (w/v) bromophenolblue, $0.5 \%$ (v/v) IPG buffer, $1.2 \%$ (v/v) De-Streak Reagent (Olsson et al. 2002) (GE Healthcare Bio-Sciences)]. For the experiments $220 \mu \mathrm{g}$ from each sample were used. For isoelectric focussing the IPGphor II with a manifold ceramic tray (GE Healthcare Bio- 
Sciences) was used. The separation in the second dimension was realised on the Ettan DALT System (GE Healthcare Bio-Sciences) with lab cast $1.0 \mathrm{~mm}$ SDS polyacrylamide gels [12.5\% (w/v)] containing Rhinohide as a gel strengthener (Molecular Probes, Leiden, NL): 20 mA/gel for minigels and $30 \mathrm{~min} 30 \mathrm{~W}, 4 \mathrm{~h} 100 \mathrm{~W}$ for six Ettan Dalt gels. Protein standard Mark 12 was purchased from INVITROGEN (Karlsruhe, Germany).

\subsubsection{Visualisation of proteins by Coomassie Brilliant Blue staining (CBB)}

Spots were visualised by colloidal CBB staining according to the protocol of Neuhoff et al. (1988). For fixation gels were incubated in fixing solution consisting of $40 \%(\mathrm{v} / \mathrm{v})$ methanol, $7 \%(\mathrm{v} / \mathrm{v})$ glacial acetic acid by incubation for two hours. Afterwards the gels were washed twice with ultrafiltrated water for $20 \mathrm{~min}$ and stained with staining solution containing $10 \%$ (w/v) methanol, $0.1 \%(\mathrm{w} / \mathrm{v})$ CBB G-250, $1.6 \%$ (v/v) o-phosphoric acid and 10\% (w/v) ammonium sulfate over night. Stained gels were neutralised by incubation in a solution containing $0.1 \mathrm{M}$ Tris-base titrated with o-phosphoric acid to a $\mathrm{pH}$ at 6.5 and incubated for 5 min. Afterwards the gels were washed in $25 \%(\mathrm{v} / \mathrm{v})$ methanol for $1 \mathrm{~h}$ and stored in the methanol solution at $4^{\circ} \mathrm{C}$.

\subsubsection{DIGE experiments, analysis and identification of spots}

For DIGE experiments five gels were made by using two biological replicates. Samples of the wildtype and the deletion strain were prepared according to Kniemeyer et al. (2006) with some modifications as mentioned above. Afterward the samples were adjusted at a $\mathrm{pH}$ of 8.5 by addition of $100 \mathrm{mM} \mathrm{NaOH}$. For labelling CyDye minimal dyes according to the manufacturer's protocol (GE Healthcare Bio-Sciences) were used. $50 \mu \mathrm{g}$ protein of each sample were labelled with $300 \mathrm{pm}$ of CyDye. Samples of both strains were labelled either with $\mathrm{Cy} 5$ or Cy3. A pool of both (wild-type and $\triangle c p c A$ ) was labelled with $\mathrm{Cy} 2$ for internal standard. Samples were mixed and incubated in the dark on ice for $30 \mathrm{~min}$. The reaction was stopped by adding $1 \mu \mathrm{l}$ of $10 \mathrm{mM}$ L-lysine. An equal volume of $4 \mathrm{x}$ sample buffer (same components as for the lysis buffer mentioned above, plus 3.2\% [vol/vol] ampholytes and 40 $\mathrm{mM}$ dithiothreitol) was added. $150 \mu \mathrm{g}$ of each labelled preparations were loaded for the first dimension on a ceramic tray by using the IPGphor II (see description above) using $24 \mathrm{~cm}$ stripes with a pH range from 3-11 (GE Healthcare Bio-Sciences). Gels were scanned by a Typhoon 9410 scanner using a resolution of $100 \mu \mathrm{m}$ and analysed with the DeCyder software (GE Healthcare). Afterwards gels were stained with coomassie as mentioned above. Spots with a regulation with at least 1.4 fold were cut out by manual and digested with trypsine for 
mass spectrometry described by Shevchenko et al. (1996). Tryptic peptides extracted from each gel slice were injected onto a reversed-phase liquid chromatograohic column (Dionex NAN75-15-03-C18 PM) by using a HPLC system from Dionex, Amsterdam, Netherlands. For mass analysis a LCQ DecaXP mass spectrometer (Thermo Electron Corp., San Jose) CA9 equipped with a nanoelectrospray ion source was used. Annotation was done by using the database of TIGR. 


\subsection{Results and Discussion}

Previous works had demonstrated the involvement of the transcription factor $\mathrm{CpcA}$ in pathogenicity (Krappmann et al., 2004). In addition, data had been presented leading to the suggestion that the basal expression level of CpcA seems to be sufficient for pathogenicity, so that an induced CPC is not crucial for virulence of A. fumigatus. To identify the "basal proteome" generated by this transcriptional activator, the proteome of wild-type strain D141 was compared to the one of the $\Delta c p c A$ deletion strain AfS01 under non-starvation conditions.

\subsubsection{Differentially expressed proteins in dependency of basal CpcA expression}

Five independent multiplex DIGE experiments of two different biological replicates were performed, whereas one of these gels represents three normal gels resulting in 15 different kinds of comparable gels for this study. After matching and quantification of all gels by using the DeCyder software, 1574 spots could be detected that appeared to be induced. For restriction conditions a cut-off at 1.4 fold or higher was set for the regulated spots. In addition, only spots that show modulation in at least 12 of the 15 samples were used for further investigation. Also signals with a t-value higher than 0.05 or without any value were not considered in our studies. 64 regulated spots remained after this restrictive procedure, but 41 of them were not picked because their signals were too weak after a Coomassie staining or the spots could not be clearly localised, so in the end 23 differentially regulated signals were cut out from the gels for mass spectrometry analysis (fig. 4.1). Analysis of these extracted putative gene products retrieved 22 different proteins, with one of them being found twice (spot numbers 1327 and 1329). In this data set only seven spots display an increased expression level in the $\Delta c p c A$ strain; the remaining 16 spots showed a higher level in the wildtype strain. All detected and analysed proteins have no higher amount than 1.81 fold (table 4.1). Altogether it can be said that the picked and analysed spots from this data set show only a modest regulation, however as a result of the restriction conditions it can be assumed that these proteins are part of the basal CPC proteome. 


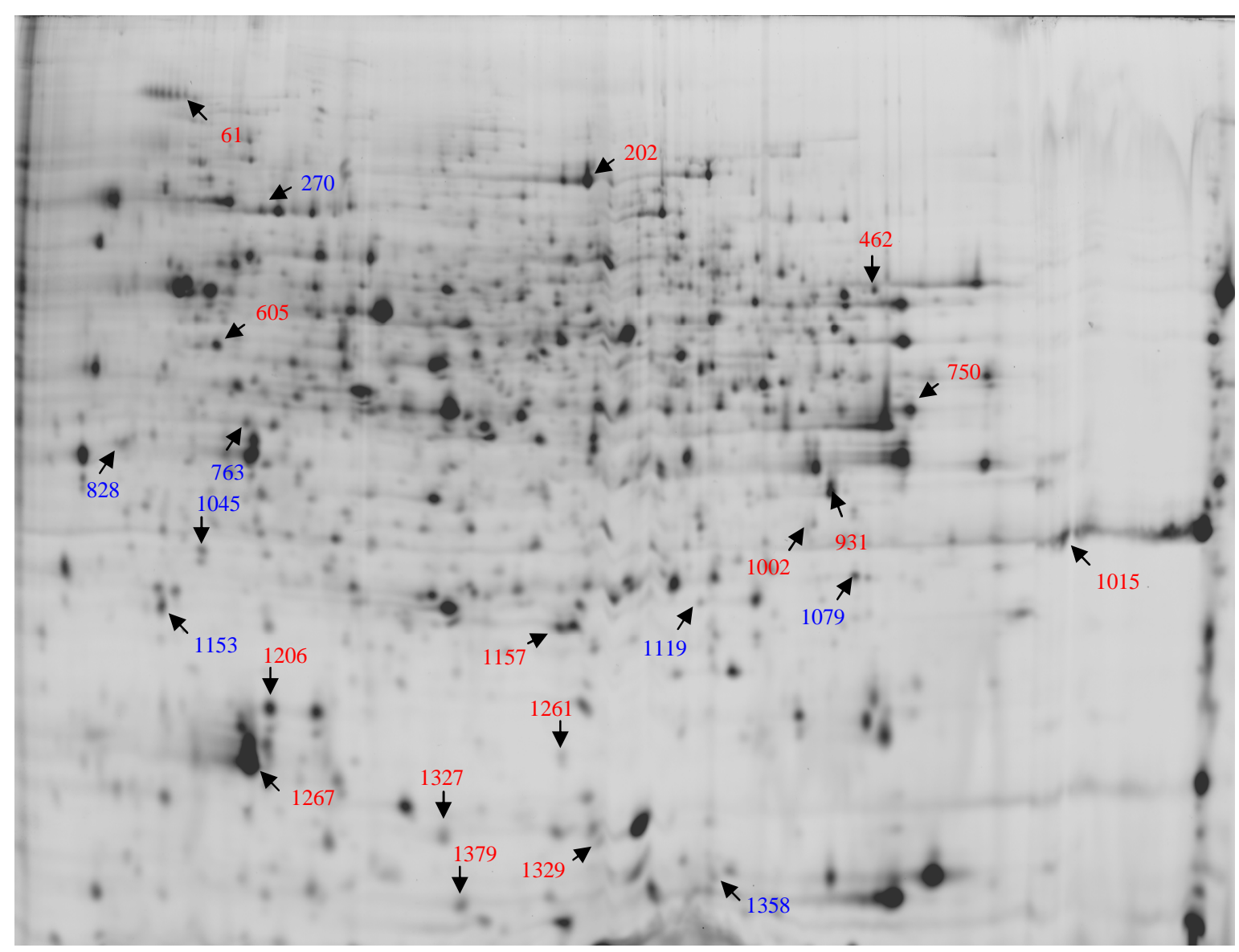

Figure 4.1. Detected spots from the DIGE after staining with Coomassie Brilliant Blue.

The figure shows all 23 detected and analysed proteins. The arrow shows the corresponding spots of every number. Up-regulated proteins of the wildtype in comparison to the $c p c A$ deletion strain have red coloured numbers and down regulated are displayed by blue numbers.

Table 4.1. Detected and identified proteins

\begin{tabular}{|l|c|c|}
\hline Detected protein & Pos. & Av. Ratio* \\
\hline Afu4g12450 pH domain protein & 61 & 1.81 \\
\hline Afu5g01440 allergen putative & 1329 & 1.70 \\
\hline Afu5g06240 alcohol dehydrogenase & 750 & 1.65 \\
\hline Afu3g11710 saccharopine dehydrogenase, putative & 605 & 1.60 \\
\hline Afu7g05660 elongation factor EF-3, putative & 202 & 1.60 \\
\hline Afu2g03140 peptide methionine sulfoxide reductase & 1261 & 1.56 \\
\hline Afu1g09930 glycerol dehydrogenase Gcy1 & 931 & 1.53 \\
\hline Afu6g02280 allergen Asp F3 & 1267 & 1.53 \\
\hline Afu8g05320 mitochondrial F1 ATPase subunit alpha & 162 & 1.51 \\
\hline Afu3g00590 Aegerolysin family protein, identical to Asp hemolysin & 1157 & 1.50 \\
\hline Afu6g10260 aldehyde reductase (AKR1) & 1002 & 1.41 \\
\hline Afu6g11620 formyltetrahydrofolate deformylase & 1015 & 1.45 \\
\hline Afu4g06910 outer mitochondrial membrane protein porin & & 1.48 \\
\hline
\end{tabular}


Table 4.1 continued.

\begin{tabular}{|l|c|c|}
\hline Detected protein & Pos. & Av. Ratio* \\
\hline Afu2g03010 cytochrome c subunit & 1206 & 1.44 \\
\hline Afu1g16840 TCTP domain protein & 1153 & -1.41 \\
\hline Afu8g03930 Hsp70 chaperone (HscA) & 270 & -1.42 \\
\hline Afu6g06450 proteasome component Pre4 & 1119 & -1.44 \\
\hline Afu1g05080 ribosomal protein P0 & 763 & -1.45 \\
\hline $\begin{array}{l}\text { Afu6g06750 14-3-3 family protein, similar to 14-3-3 protein important for } \\
\text { polarized growth }\end{array}$ & 828 & -1.50 \\
\hline Afu3g00730 conserved hypothetical protein & 1045 & -1.60 \\
\hline Afu5g08830 HEX1 & 1358 & -1.71 \\
\hline Afu3g07430 peptidyl-prolyl cis-trans isomerase & & -1.72 \\
\hline
\end{tabular}

*multiplication factor for regulation

\subsubsection{Groups of function of the basal proteome directed by $\mathrm{CpcA}$}

The complete set of identified proteins was classified and divided into different groups of function. Analysis of the 14 proteins that are up regulated in the wild-type leads to a preliminary categorisation in seven different classes (table 4.2). Most gene products of these analysed proteins belong to metabolic pathways. For example the alcohol dehydrogenase (Afu6g102690), an enzyme involved in fermentation, that was 1.65 fold increased in the wildtype situation. Other proteins were identified, one with locus tag Afu1g09930 encoding a glycerol dehydrogenase that showed an up-regulation of 1.53 fold in the D141 strain. Adjacent to the glycerol metabolism this protein seems to be also involved in stress response induced by salt concentrations. An aldehyde reductase (Afu6g10260) that had a 1.48 fold increased expression level was also detectable in the data as well as a formyltetrahydrofolate deformylase (Afu6g11620), which is likely required for synthesis of purine ribonucleotides. Surprisingly, just one enzyme that is involved in amino acid biosynthesis was found; this putive saccharopine dehydrogenase with the locus tag Afu3g11710 catalyses the final synthesis step for lysine.

The second largest group containing three proteins from the up-regulated data set belongs to the aerobic respiration system comprising the proteins with the locus tags Afu2g03010, Afu4g06910 and Afu8g05320. All three can be found in the mitochondrial membranes. The mitochondrial F1 ATPase subunit alpha is needed for ATP synthesis coupled with proton transport and therefore has just an indirect connection to the aerobic respiration system. The other two candidates, an outer mitochondrial membrane protein porin and the cytochrome $\mathrm{c}$ subunit $\mathrm{Vb}$, are directly involved in the respiration system. Furthermore, proteins were found 
that might play a role in pathogenicity of A. fumigatus. One of them is the allergen Asp3 for which it is known that it is a factor related to virulence. The other one is a putative allergen, for which the function in connection with virulence is not really clear. For both gene products it is known that they are needed in the regulation of cell redox homeostasis. The third one is the Asp-hemolysin. In earlier studies it was shown that hemolysin supports the infection by A. fumigatus in a mouse model and that antibodies against this factor can decrease infection in an animal model. Nevertheless, it is not a main virulence factor but it may support other proteins involved in pathogenicity (Malicev et al., 2007). The Asp-hemolysin displays an upregulation of 1.5 fold in comparison to the $\triangle c p c A$ deletion strain, leading to the suggestion that $\mathrm{CpcA}$ is necessary for expression of this protein under non-starvation conditions.

Another important protein that was found with an increased level of 1.56 fold paticipates to the reaction on oxidative stress. The corresponding locus Afu2g03140 designates a gene that encodes a putative peptide methionine sulfoxide reductase, which may be involved in the reaction on oxidative stress. Experiments in S. cerevisae had pointed out that methionine sulfoxide reductases have the ability to reverse methione oxidation (MetO) caused by reactive oxidative species (ROS) (Moskovitz et al., 2000; Moskovitz et al., 2002). A dysfunction of these proteins leads to an accumulation of carbonyl proteins and results in a higher aging in yeast cells (Oien and Moskovitz, 2007).

The last two proteins, which were identified in our studies, are a putative elongation factor (Afu7g05660) that is needed for translation and a pH domain-containing protein (Afu4g12450), which may act as a kind of chaperone or may be important to react on changing $\mathrm{pH}$ conditions.

From the eight analysed spots displaying a down-regulation in the wild-type, five of them were classified and assigned to functional categories (table 4.2). Three are hypothetical proteins or proteins with unknown function so that a disposition in a special functional category was not possible. In addition the heat shock protein Hsp70 chaperone (HscA) having the locus tag Afu8g03930 was identified. From yeast it is known that the homologue Ssb2p interacts with the Hsp70 family member Ssz1p and the J-domain protein Zuo1p. These three chaperones form a functional triad required for translation. Deletion of one of these components leads to different dysfunctions and to a reduced growth in S. cerevisiae (Conz et al., 2007; Gautschi et al., 2002; Hundley et al., 2002). Other chaperones were not found in our studies. Proteins involved in protein metabolism and anabolism were also found in this proteomic experiments: the proteasome component Pre4, a subunit of the 20S proteasome concerned in the ubiquitin dependent protein catabolism displays down-regulation in the wild- 
type. Moreover, a peptidyl-prolyl cis-trans isomerase putatively involved in protein metabolism was identified. Only one spot was identified as a protein involved in signal transduction and which belongs to the 14-3-3 family. These are dimeric, acidic proteins having an average size of $30 \mathrm{kDa}$ that are highly conserved and can be found in all investigated eukaryotes. The number of these proteins differs from organism to organism and can range from one up to thirteen isoforms within one organism. In S. cerevisiae and S. pombe for example only two members of 14-3-3 family are described, whereas in $A$. thaliana thirteen of these proteins exist (van Heusden and Steensma, 2006). In contrast to this the pathogenic yeast C. albicans contains only one of these proteins (Cognetti et al., 2002). Their function is very manifold so that the 14-3-3 proteins play a role in many different mechanisms and pathways resulting in many different interaction partners. The involvement in DNA repair, which was shown by Ford et al. (1994), is only one example. In C. albicans the single isoform of 14-3-3 proteins is important for filamentous growth and therefore for pathogenicity. Without the corresponding gene an invasive growth seems to be impossible for this organism (Cognetti et al., 2002; Hurtado and Rachubinski, 2002). As a result of this complexity the research and analysis of this family is very challenging.

Table 4.2. Classification of the different proteins found in the DIGE experiments

\begin{tabular}{|c|c|}
\hline Classification & Av. Ratio \\
\hline \multicolumn{2}{|l|}{ metabolism } \\
\hline Afu6g10260 aldehyde reductase (AKR1) & 1.48 \\
\hline Afu3g11710 saccharopine dehydrogenase, putative & 1.60 \\
\hline Afu1g09930 glycerol dehydrogenase Gcy1 & 1.53 \\
\hline Afu5g06240 alcohol dehydrogenase & 1.65 \\
\hline Afu6g11620 formyltetrahydrofolate deformylase & 1.41 \\
\hline \multicolumn{2}{|l|}{ aerobic respiration } \\
\hline Afu2g03010 cytochrome c subunit & 1.44 \\
\hline Afu4g06910 outer mitochondrial membrane protein porin & 1.45 \\
\hline Afu8g05320 mitochondrial F1 ATPase subunit alpha & 1.51 \\
\hline \multicolumn{2}{|l|}{ oxidative stress } \\
\hline Afu2g03140 peptide methionine sulfoxide reductase & 1.56 \\
\hline \multicolumn{2}{|l|}{$\mathrm{pH}$ regulation } \\
\hline Afu4g12450 PH domain protein & 1.81 \\
\hline \multicolumn{2}{|l|}{ pathogenesis } \\
\hline Afu3g00590 Aegerolysin family protein, identical to Asp hemolysin & 1.50 \\
\hline Afu5g01440 allergen putative & 1.70 \\
\hline Afu6g02280 allergen Asp F3 & 1.57 \\
\hline
\end{tabular}


Table 4.2 continued.

\begin{tabular}{|l|c|}
\hline Classification & Av. Ratio \\
\hline translation & \\
\hline Afu7g05660 elongation factor EF-3, putative & 1.60 \\
\hline heat shock proteins & \\
\hline Afu8g03930 Hsp70 chaperone (HscA) & -1.42 \\
\hline translation & \\
\hline Afu1g05080 ribosomal protein P0 & -1.45 \\
\hline signal transduction & \\
\hline Afu6g06750 14-3-3 family protein, similar to 14-3-3 protein important for polarized growth; & -1.50 \\
\hline protein degradation and metabolism & -1.72 \\
\hline Afu3g07430 peptidyl-prolyl cis-trans isomerase & -1.44 \\
\hline Afu6g06450 proteasome component Pre4 & \\
\hline unknown function & -1.60 \\
\hline Afu3g00730 conserved hypothetical protein & -1.41 \\
\hline Afu1g16840 TCTP domain protein & -1.71 \\
\hline Afu5g08830 HEX1 & \\
\hline
\end{tabular}

In summary in this particular study a variety of different proteins were found, which do not belong to one special functional group. In a next step it should be validated whether these proteins are direct targets of CpcA. For this purpose an analysis of the 5' upstream region was made for each of the 22 detected genes with the aim to reveal putative CpcA binding sites.

\subsubsection{Presence of putative CpcA binding sites}

CpcA belongs to the leucine zipper (bZIP) family of transcriptional activators and has the ability to bind to specific recognition sites called GCREs or CPREs (General Control or Cross-Pathway Control Recognition Elements). The basic region of these proteins consists of a pair $\alpha$ helices required for binding to the target DNA (Landschulz et al., 1988; Struhl, 1989). For Gcn $4 p$ in $S$. cerevisiae it was shown that there is a high affinity to the canonical sequence 5'-TGACTCA-3' leading to strong binding that results in a direct regulation of the corresponding gene (Arndt and Fink, 1986; Hinnebusch, 1984; Natarajan et al., 2001; Thireos et al., 1984). This sequence as the recognition site of Gcn4p is highly conserved and can be found in other organisms like filamentous fungi. In A. nidulans this recognition sequence was detected and identified as a binding site for CpcA, the homologue of Gcn $4 p$ (Hoffmann et al., 2001). It was possible to find this sequence in the promoter region of the $c p c A$ gene. Studies in other filamentous fungi like $N$. crassa, for which also this recognition sequence was found in the 5' upstream region of the GCN4-corresponding gene $c p c-1$, had shown that this 
sequence enables an autoregulation of the transcriptional regulator (Paluh et al., 1988). Later, the same was shown for A. nidulans (Hoffmann et al., 2001) leading to the suggestion that this autoregulation is also the case in A. fumigatus because there is the same recognition sequence in the $c p c A$ promoter. Further detailed studies in $N$. crassa and $S$. cerevisiae had displayed that small variations in the canonical binding site also support interaction with the transcriptional activator $\mathrm{CPC}-1 / \mathrm{Gcn} 4 \mathrm{p}$. Given the high similarity between $\mathrm{CpcA}$ and its homologues in the other organisms it is not surprisingly that these short sequences are also detectable in A. fumigatus, where they represent putative binding targets for the $c p c A$ gene product. In this study the promoter regions within 1000 basepairs of genes corresponding to the proteins detected in the proteomic analysis were analysed for presence of the canonical sequence 5'-TGACTCA-3' and three further putative binding sites (fig. 4.2). Although the affinity of the transcriptional activator to these three diverging recognition elements is not as high as to the original one, previous works showed that the possibility of binding is still increased in contrast to other sequences (Tian et al., 2007).

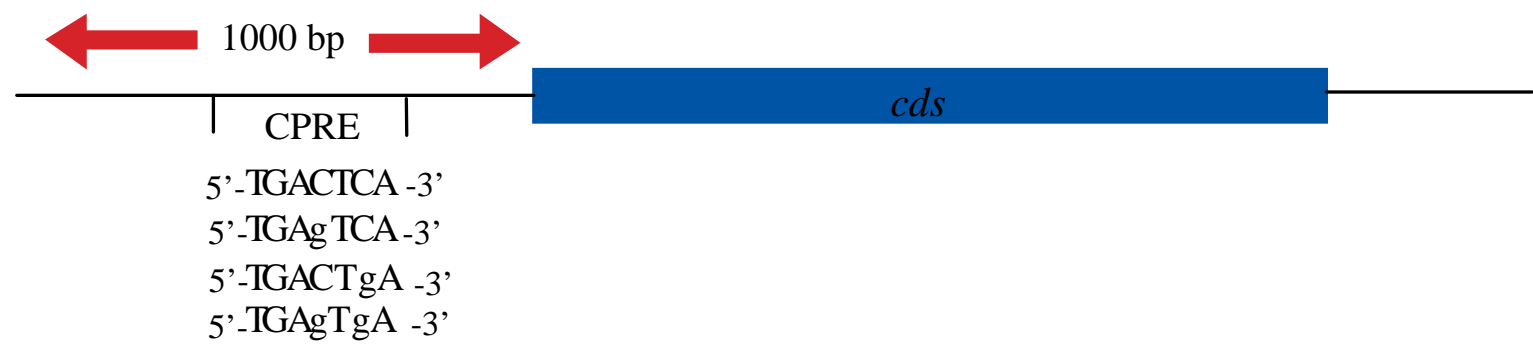

Figure 4.2. Putative CpcA binding sites

Investigation of the promoter region within 1000 basepairs for putative CpcA binding sites of the analysed proteins. Four target sequences were checked. The first one is the canonical sequence with the highest affinity to CpcA.

Investigation of the promoter regions of the genes resulting from the corresponding proteins of the proteomic data set yielded to the result that eight genes have one of the four checked sequences within their promoter region (table 4.3). Five of them are up-regulated proteins in the data set and three are down-regulated. Only one of the five up-regulated genes which encodes the putative allergen with the locus tag Afu5g01440 contains the high affinity binding site 5'-TGACTCA-3', all others have the less specific binding sequences in their promoter regions. In three upstream regions the 5'-TGAgTgA-3' sequence was found containing two mismatches from $\mathrm{C}$ to $\mathrm{G}$ at the fourth and sixth positions. The gene with the locus tag Afu3g11710 encoding the saccharopine dehydrogenase is the only protein having in its promoter the sequence 5'-TGAgTCA-3' as putative binding region for CpcA. The sequence 5'-TGACTgA-3' does not appear in any of these genes. In all three down-regulated 
genes the typical CpcA binding region is missing whereas the sequence 5'-TGAgTCA-3' and once 5'-TGACTgA-3' was detected twice. Promoter regions with two or more regulation elements could not be found.

Table 4.3. Proteins of the proteomic experiments containing a putative CpcA binding site

\begin{tabular}{|l|l|}
\hline Gene & \multicolumn{1}{|c|}{ CPRE } \\
\hline Afu3g11710 saccharopine dehydrogenase, putative & 5'-TGAGTCA-3' \\
\hline Afu6g11620 formyltetrahydrofolate deformylase & 5'-TGAGTGA-3' \\
\hline Afu3g00590 Aegerolysin family protein, identical to Asp hemolysin & 5' 'TGAGTGA-3' \\
\hline Afu5g01440 allergen putative & 5'-TGACTCA-3' \\
\hline Afu7g05660 elongation factor EF-3, putative & 5'-TGAGTGA-3' \\
\hline Afu8g03930 Hsp70 chaperone (HscA) & 5'-TGACTGA-3' \\
\hline Afu3g00730 conserved hypothetical protein & 5' '-TGAGTCA-3' \\
\hline Afu1g16840 TCTP domain protein & 5'-TGAGTCA-3' \\
\hline
\end{tabular}

Finally, a comparison of transcriptomic data monitored for the transcriptional activator CpcA under amino acid starvation conditions (see Chapter 1) with the proteomic data set was carried out to detect proteins, which are also regulated on transcriptional level under starvation conditions. Only two proteins could be found, which were regulated in both experiments: the aldehyde reductase (AKR1) and the putative saccharopine dehydrogenase, both involved in metabolism.

To summarize these results it can be stated that proteins involved in different cellular functions depend in their expression level under non-starvation conditions on the CPC activator CpcA. However, their significance for pathogenicity of A. fumigatus, especially with respect to the necessity of the Cross-Pathway Control transcriptional activator $\mathrm{CpcA}$, remains to be demonstrated. A prime candidate for further research is the Asp hemolysin-encoding gene. For this, a significant dependency of expression on $\mathrm{CpcA}$ is evident and the corresponding promoter region comprises a conserved, high affinity binding site for this activator. Accordingly, absence of $\mathrm{CpcA}$ results in reduced levels of Asp hemolysin due to the fact that no binding and activation at the promoter of the encoding gene occurs. Yet, further confirmatory studies will have to be carried out in order to substantiate this. 


\subsection{References}

Arndt, K., and Fink, G.R. (1986) GCN4 protein, a positive transcription factor in yeast, binds general control promoters at all 5' TGACTC 3' sequences. Proc Natl Acad Sci U S A 83: $8516-8520$.

Brakhage, A.A., and Langfelder, K. (2002) Menacing mold: the molecular biology of Aspergillus fumigatus. Annu Rev Microbiol 56: 433-455.

Braus, G.H., Pries, R., Düvel, K., Valerius, O. (2004) Molecular Biology of Fungal Amino Acid Biosynthesis Regulation. In Kück $U(e d)$, The Mycota II, Genetics and Biotechnology, 2nd edn. Springer Press, Berlin Heidelberg New York Tokyo: 239-269.

Bromley, I.M., and Donaldson, K. (1996) Binding of Aspergillus fumigatus spores to lung epithelial cells and basement membrane proteins: relevance to the asthmatic lung. Thorax 51: 1203-1209.

Burns, C., Geraghty, R., Neville, C., Murphy, A., Kavanagh, K., and Doyle, S. (2005) Identification, cloning, and functional expression of three glutathione transferase genes from Aspergillus fumigatus. Fungal Genet Biol 42: 319-327.

Chang, Y.C., Tsai, H.F., Karos, M., and Kwon-Chung, K.J. (2004) THTA, a thermotolerance gene of Aspergillus fumigatus. Fungal Genet Biol 41: 888-896.

Cognetti, D., Davis, D., and Sturtevant, J. (2002) The Candida albicans 14-3-3 gene, BMH1, is essential for growth. Yeast 19: 55-67.

Conz, C., Otto, H., Peisker, K., Gautschi, M., Wolfle, T., Mayer, M.P., and Rospert, S. (2007) Functional characterization of the atypical Hsp70 subunit of yeast ribosome-associated complex. J Biol Chem 282: 33977-33984.

Cooney, D.G., Emerson, R., (1964) Thermophilic Fungi. An Account of their Biology, Avtivities and Classification. W.H. Freeman, San Francico, CA.

Gautschi, M., Mun, A., Ross, S., and Rospert, S. (2002) A functional chaperone triad on the yeast ribosome. Proc Natl Acad Sci U S A 99: 4209-4214.

Gil, M.L., Penalver, M.C., Lopez-Ribot, J.L., O'Connor, J.E., and Martinez, J.P. (1996) Binding of extracellular matrix proteins to Aspergillus fumigatus conidia. Infect Immun 64: 5239-5247.

Hinnebusch, A.G. (1984) Evidence for translational regulation of the activator of general amino acid control in yeast. Proc Natl Acad Sci U S A 81: 6442-6446.

Hoffmann, B., Valerius, O., Andermann, M., and Braus, G.H. (2001) Transcriptional autoregulation and inhibition of mRNA translation of amino acid regulator gene $c p c A$ of filamentous fungus Aspergillus nidulans. Mol Biol Cell 12: 2846-2857.

Hundley, H., Eisenman, H., Walter, W., Evans, T., Hotokezaka, Y., Wiedmann, M., and Craig, E. (2002) The in vivo function of the ribosome-associated Hsp70, Ssz1, does not require its putative peptide-binding domain. Proc Natl Acad Sci U S A 99: 42034208.

Hurtado, C.A., and Rachubinski, R.A. (2002) YlBMH1 encodes a 14-3-3 protein that promotes filamentous growth in the dimorphic yeast Yarrowia lipolytica. Microbiology 148: 3725-3735.

Krappmann, S., Bignell, E.M., Reichard, U., Rogers, T., Haynes, K., and Braus, G.H. (2004) The Aspergillus fumigatus transcriptional activator CpcA contributes significantly to the virulence of this fungal pathogen. Mol Microbiol 52: 785-799.

Landschulz, W.H., Johnson, P.F., and McKnight, S.L. (1988) The leucine zipper: a hypothetical structure common to a new class of DNA binding proteins. Science 240: 1759-1764.

Latgé, J.P. (1999) Aspergillus fumigatus and aspergillosis. Clin Microbiol Rev 12: 310-350.

Maheshwari, R., Bharadwaj, G., and Bhat, M. K. (2000) Thermophilic fungi: their physiology and enzymes. Microbiol Mol Biol Rev 64: 461-488. 
Malicev, E., Chowdhury, H.H., Macek, P., and Sepcic, K. (2007) Effect of ostreolysin, an Asp-hemolysin isoform, on human chondrocytes and osteoblasts, and possible role of Asp-hemolysin in pathogenesis. Med Mycol 45: 123-130.

May, G.S., Xue, T., Kontoyiannis, D.P., and Gustin, M.C. (2005) Mitogen activated protein kinases of Aspergillus fumigatus. Med Mycol 43 Suppl 1: S83-86.

Moskovitz, J., Poston, J.M., Berlett, B.S., Nosworthy, N.J., Szczepanowski, R., and Stadtman, E.R. (2000) Identification and characterization of a putative active site for peptide methionine sulfoxide reductase (MsrA) and its substrate stereospecificity. J Biol Chem 275: 14167-14172.

Moskovitz, J., Singh, V.K., Requena, J., Wilkinson, B.J., Jayaswal, R.K., and Stadtman, E.R. (2002) Purification and characterization of methionine sulfoxide reductases from mouse and Staphylococcus aureus and their substrate stereospecificity. Biochem Biophys Res Commun 290: 62-65.

Natarajan, K., Meyer, M.R., Jackson, B.M., Slade, D., Roberts, C., Hinnebusch, A.G., and Marton, M.J. (2001) Transcriptional profiling shows that Gen4p is a master regulator of gene expression during amino acid starvation in yeast. Mol Cell Biol 21: 43474368 .

Oien, D., and Moskovitz, J. (2007) Protein-carbonyl accumulation in the non-replicative senescence of the methionine sulfoxide reductase A (msrA) knockout yeast strain. Amino Acids 32: 603-606.

Paluh, J.L., Orbach, M.J., Legerton, T.L., and Yanofsky, C. (1988) The cross-pathway control gene of Neurospora crassa, cpc-1, encodes a protein similar to GCN4 of yeast and the DNA-binding domain of the oncogene v-jun-encoded protein. Proc Natl Acad Sci U S A 85: 3728-3732.

Penalver, M.C., O'Connor, J.E., Martinez, J.P., and Gil, M.L. (1996) Binding of human fibronectin to Aspergillus fumigatus conidia. Infect Immun 64: 1146-1153.

Philippe, B., Ibrahim-Granet, O., Prevost, M.C., Gougerot-Pocidalo, M.A., Sanchez Perez, M., Van der Meeren, A., and Latgé, J.P. (2003) Killing of Aspergillus fumigatus by alveolar macrophages is mediated by reactive oxidant intermediates. Infect Immun 71: 3034-3042.

Reuss, O., and Morschhauser, J. (2006) A family of oligopeptide transporters is required for growth of Candida albicans on proteins. Mol Microbiol 60: 795-812.

Sasse, C., Bignell, E.M., Hasenberg, M., Haynes, K., Gunzer, M., Braus, G.H., and Krappmann, S. (2008) Basal expression of the Aspergillus fumigatus transcriptional activator CpcA is sufficient to support pulmonary aspergillosis. Fungal Genet Biol.

Soubani, A.O., and Chandrasekar, P.H. (2002) The clinical spectrum of pulmonary aspergillosis. Chest 121: 1988-1999.

Staib, F., Mishra, S.K., Rajendran, C., Voigt, R., Steffen, J., Neumann, K.H., Hartmann, C.A., and Heins, G. (1980) A notable Aspergillus from a mortal aspergilloma of the lung. New aspects of the epidemiology, serodiagnosis and taxonomy of Aspergillus fumigatus. Zentralbl Bakteriol A 247: 530-536.

Struhl, K. (1989) Helix-turn-helix, zinc-finger, and leucine-zipper motifs for eukaryotic transcriptional regulatory proteins. Trends Biochem Sci 14: 137-140.

Thireos, G., Penn, M.D., and Greer, H. (1984) 5' untranslated sequences are required for the translational control of a yeast regulatory gene. Proc Natl Acad Sci U S A 81: 50965100.

Tian, C., Kasuga, T., Sachs, M.S., and Glass, N.L. (2007) Transcriptional Profiling of Cross Pathway Control in Neurospora crassa and Comparative Analysis of the Gcn 4 and CPC1 Regulons. Eukaryot Cell 6: 1018-1029. 
Tronchin, G., Bouchara, J.P., Larcher, G., Lissitzky, J.C., and Chabasse, D. (1993) Interaction between Aspergillus fumigatus and basement membrane laminin: binding and substrate degradation. Biol Cell 77: 201-208.

van Heusden, G.P., and Steensma, H.Y. (2006) Yeast 14-3-3 proteins. Yeast 23: 159-171. 


\section{Chapter 5}

\section{Conclusions \& Outlook}

\subsection{Conclusions \& Outlook}

Pathogenic organisms like the filamentous fungi $A$. fumigatus require pathways and mechanisms for adaptation within the host. A better understanding of these regulatory systems make easier to find new targets for antifungal drugs and pharmaceutica. One of these systems is the Cross-Pathway Control (CPC), which is the homologous to the well-studied General Control of amino acid biosynthesis (GC) (Deng et al., 2004; Hannig and Hinnebusch, 1988; Hannig et al., 1990; Hinnebusch, 2005). In its core, this global regulatory system contains a sensor kinase (CpcC) and a transcriptional regulator (CpcA) (Hoffmann et al., 2001), for which it was demonstrated that it is required for full virulence of A. fumigatus (Krappmann et $a l .$, 2004). Therefore, the main focus of this work was set on the CPC with its components $\mathrm{CpcC}$ and $\mathrm{CpcA}$. For sure a better understanding of this regulatory mechanism enables a more detail insight of a pathogenic filamentous fungus resulting in increasing knowledge that can give hints for new therapies against this pathogen.

\subsubsection{The CPC is a global network required for many different stress responses}

In the first part of this work it was the aim to find out what kind of genes are under the regulation of the transcriptional regulator $\mathrm{CpcA}$ and so are parts of the $\mathrm{CPC}$ regulatory network. Microarray hybridisation experiments were carried out as experimental approach to get a comprehensive overview. 523 putative CpcA targets with a regulated mRNA level of at least 1.5 fold were detected, with 377 genes being up-regulated in this data set and only 146 being repressed under the applied conditions (for an overview see fig. 5.2). By the finding that several transcriptional regulators are induced under starvation it becomes clear that the CrossPathway Control is a global regulatory system acting on additional factors and regulators to produce a fast and strong response to incoming environmental signals. It could also be confirmed that the CPC is involved in the regulation of different metabolism pathways as it has been shown for other fungi like yeast or N. crassa (Natarajan et al., 2001; Tian et al., 2007). In addition to many amino acid biosynthesis genes also a high number of transporters and permeases are up-regulated in our data set. Some of them are not required for metabolism but involved in pumping toxic molecules out of the fungus. This illustrated the requirement of 
the system for detoxification of different drugs by channelling them via transport mechanisms out of the organism and not only to react on starvation condition like amino acid limitation or glucose and purine starvation (Rolfes and Hinnebusch, 1993; Yang et al., 2000). The detection of putative stress response genes involved in neutralisation of oxidative reactive species and others, which confer the fungus a higher resistance to different kind of drugs e.g. itraconazole corroborates the suggestion that the CPC control is an important stress response system acting widely on the cellular level. Additionally, two genes encoding proteins of the AAA-ATPases family ( $c d a A$ and $c d a B$ ) could be identified on which our further experiments were based. The family of the AAA-ATPases (․ㅡPases associated with various cellular activities) can be found in all kingdoms and show a diversity of functions in every organism. The core of the AAA proteins is an ATP- binding domain, which contains about 200 to 250 amino acids. In earlier studies it was possible to localise the different parts of the AAA proteins by crystal structure analysis and to detect oligomers of AAA ATPases, which normally occurs in hexamers (Hanson and Whiteheart, 2005). In general the proteins have six different domains, which give them the typical characterisation. Most of these proteins undergo a conformation and or bind ATP for hydrolysation resulting in physical works. But the exactly mechanism of changing the conformation is still not completely understand. The functions of these proteins range from reactions during replication via protein folding and degradation to processes in which chaperones are involved. But till now the function of many AAA-ATPases is still unclear and more effort will be needed to get a better and more understandable overview of this group of proteins (Davey et al., 2002; Hanson and Whiteheart, 2005; Wang et al., 2004). Analysing genomic databases of different organisms showed that most of the eukaryotes contain 50-80 members of this family. Arabidopsis thaliana is an exception with a number of circa 140 AAA-ATPases. Resulting from this the interest was to find out how many of these proteins are exist in A. fumigatus via blasting the AAA-motif sequence against the genome of this fungus, by which only eleven putative proteins were retrieved.

After validation via Northern hybridisation that $\mathrm{CpcA}$ regulates both $c d a$ genes their cellular function was investigated by phenotypic characterisation of deletion mutant strains. From this, a putative involvement in resistance to conditions evoking cell wall stress was deduced. In addition, induction of $\mathrm{CpcA}$ by Calcofluor White treatment but not activation of the whole CPC system was evident. Therefore, it can be suggested that $\mathrm{CpcA}$ as the $\mathrm{CPC}$ transcriptional regulator can also be induced independently from this global system via different stress signals. Another hint for this suspicion is given by comparison of the amino acid starvation 
data set with the transcriptional profile of A. fumigatus under antifungal stress induced by voriconazole (da Silva Ferreira et al., 2006). After $4 \mathrm{~h}$ incubation with voriconazole the data set show an increased transcriptional level of CpcA as well as the two $c d a$-encoded AAAATPases but not of typical amino acid biosynthesis genes like $\arg B$, which would indicate an induction of the CPC. Interestingly, no induction of the other nine AAA-ATPases in either of these data sets was evident. Therefore, it can be assumed that these two AAA-ATPases (CdaA and $\mathrm{CdaB}$ ) are the only genes of this family regulated by $\mathrm{CpcA}$. It will be interesting for further studies to analyse the functions of these proteins in more detail. In addition, analysis of other putative stress genes from the amino acid starvation data would be very interesting, especially in relation to drug response and reaction to oxidative stress.

\subsubsection{The CPC is not triggered during the beginning of infection}

Previous works had supported a role of CpcA in A. fumigatus pathogenicity (Krappmann et $a l .$, 2004). Based on this finding it was the aim of this work to gain more information on this transcriptional activator during infection. A $g f p:: c p c A$ reporter strain was constructed to carry out in vitro confrontations with macrophages isolated from cell lines and mice. Interactions between these immune defence cells and conidia of A. fumigatus did not result in induction of CpcA expression, and additional in vivo experiments supported this observation. Accordingly, these experiments demonstrate that during the first period of infection the CPC system is not triggered, leading to the suggestion that starvation conditions for e.g. amino acid or glucose limitation do not exist in the murine lung or respectively in macrophage phagosomes (as overview see fig. 5.1). This result is supported by earlier studies in which proteomic experiments of the human pathogenic yeast $C$. albicans show an increased level of Gcn $4 p$ during ingestion by neutrophils but not by macrophages (Fernandez-Arenas et al., 2007).

Furthermore, the sensor kinase of A. fumigatus required for the regulation of $\mathrm{CpcA}$ expression was characterised in detail. The function and the mechanism of this protein were described in earlier works for S. cerevisiae, N. crassa and A. nidulans (Carsiotis et al., 1974; Davis, 2000; Hinnebusch, 1984, 2005; Hoffmann et al., 2001; Piotrowska, 1980; Sachs, 1996). It found out that the deletions of $\mathrm{CpcC}$ leads to an intermediate growth phenotype in comparison to the wild-type and the $c p c A$ deletion strain on medium inducing amino acid starvation and that $\mathrm{CpcC}$ is needed for induction of the CPC in A. fumigatus. Interestingly, a CpcC deletion strain is still able to phosphorylate the eIF2 $\alpha$ subunit under amino acid starvation. The conclusion on this is that there might be another kinase with the ability to phosphorylate the initiation factor. However, it could be demonstrated that this residual phosphorylation has no effect on 
$\mathrm{CPC}$ induction by increased $\mathrm{CpcA}$ translation. In further experiments the influence of $\mathrm{CpcC}$ on fungal pathogenicity was assessed in a murine model. The results made clearly demonstrated that $\mathrm{CpcC}$, in contrast to $\mathrm{CpcA}$, is not required for pathogenicity. Concluding from this and from the described microscopy experiments it can be suggested that the basal level of $\mathrm{CpcA}$ is sufficient for full virulence. Therefore it will be of interest to identify genes that depend in their transcription on the basal expression level of $\mathrm{CpcA}$ in order to get a better understanding for the infection mechanisms of this pathogenic fungus.

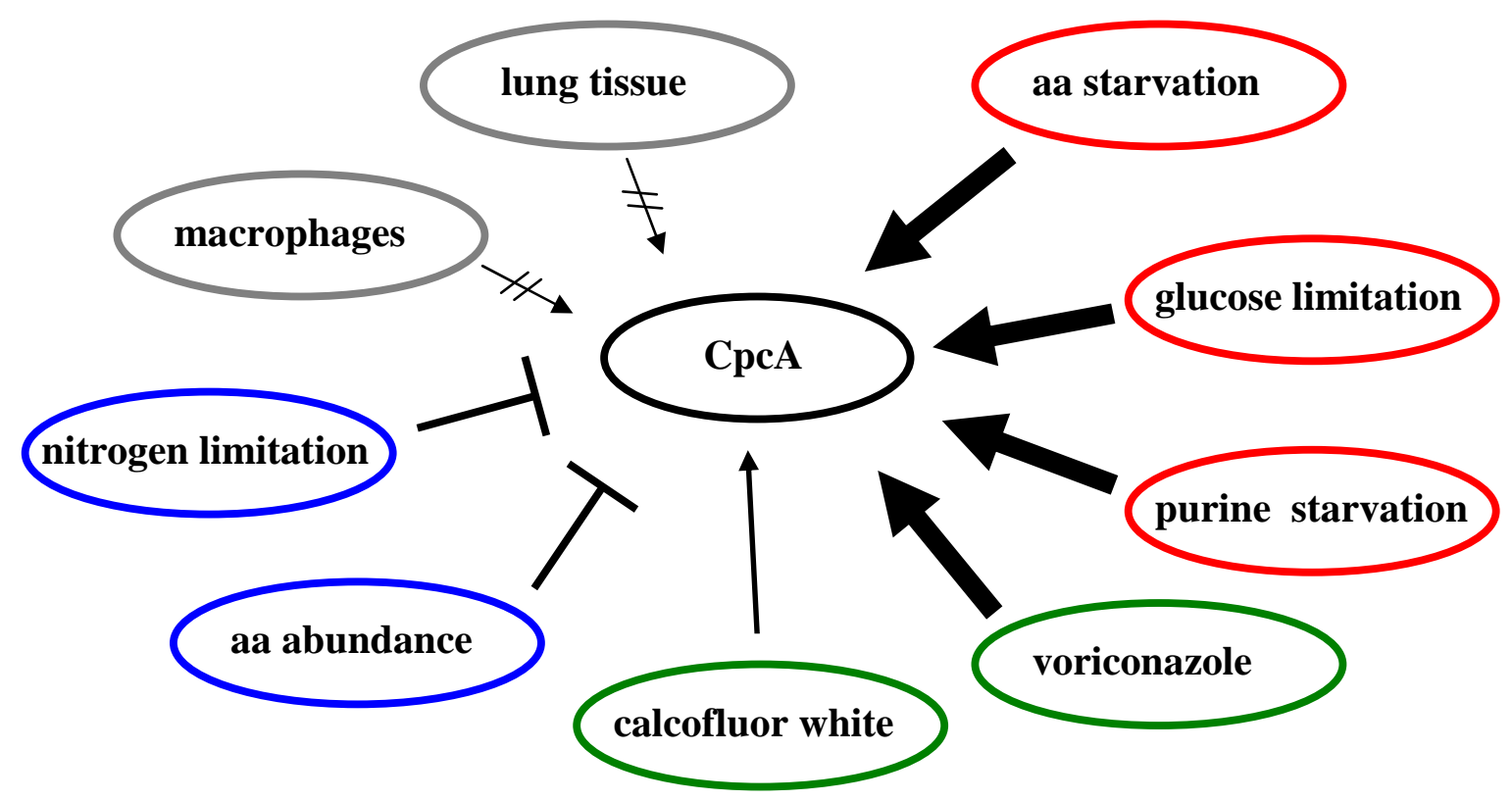

Figure 5.1. Different factors that influence the transcriptional activator $\mathrm{CpcA}$

The scheme displays all influences, which have more or less an influence on CpcA. Red-circled factors have an inductive effect on the transcriptional activator and on the CPC. In contrast to that are the green-circled influences that only induce $\mathrm{CpcA}$ but have no effect on the CPC. Big arrows present in the figure strong upregulation of $\mathrm{CpcA}$. Factors and influences that repress the induction (blue circled) or have no effect to CpcA (grey circled) are also shown in this figure.

\subsubsection{The basal level of CpcA regulates genes involved in pathogenicity}

Based on the experiments showing that in contrast to $\mathrm{CpcA} \mathrm{CpcC}$ has no influence to the virulence of A. fumigatus (Krappmann et al., 2005; Sasse et al., 2008) it was suggested that the basal expression level of transcriptional activator appears to be sufficient. In order to get a first overview on genes depending in their expression on the presence of CpcA under nonstarvation conditions, a proteomic approach was followed. 22 different spots could be detected and assigned to 22 proteins (fig. 5.2). Their functional categorisation displayed a strong diversity; nevertheless, it was possible to find three putative candidates that are likely to be involved in pathogenicity. We also discover a protein whose function is required for 
detoxification of oxidative reactive species. These results are perhaps a hint for an explanation of the A. fumigatus virulence in dependency of CpcA. In additional works we investigate the promoter regions of all 22 detected genes to find putative $\mathrm{CpcA}$ binding sites.

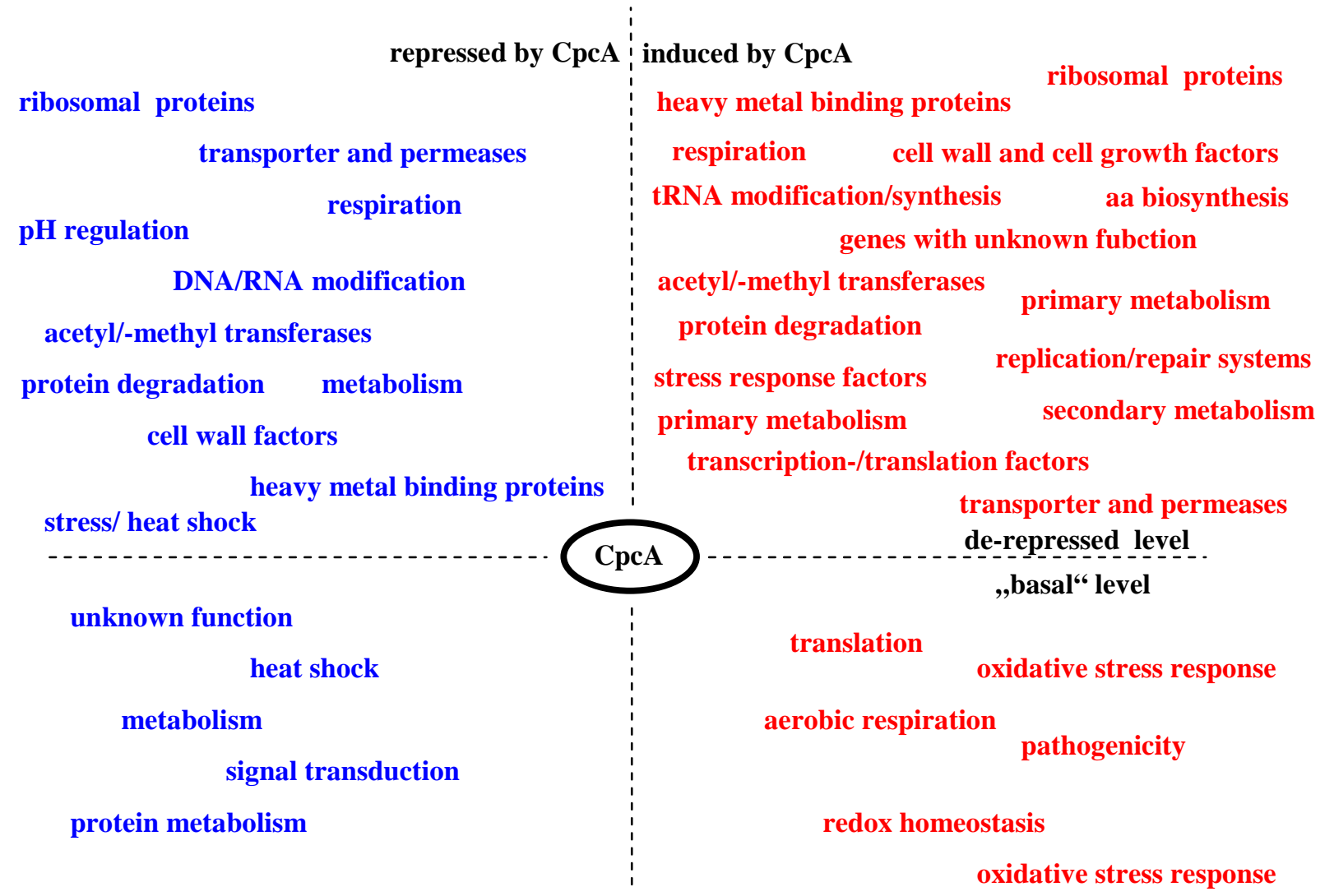

Figure 5.2. Groups of function that are regulated under starvation and non-starvation conditions in dependency of $\mathrm{CpcA}$

The figure shows all main groups that are regulated by $\mathrm{CpcA}$ directly and indirectly. Red marked groups are here the induced ones and blue the repressed genes. The lower section in this figure represents the genes regulated under non starvation conditions hereas the upper part shows the targets of $\mathrm{CpcA}$ under amino acid starvation conditions.

Although some of these genes have binding sequence for the transcriptional regulator, surprisingly just two of the regulated proteins are present in the transcriptome data set on CpcA-regulated genes (see Chapter 1), and these have a function in metabolic routes but not in virulence. It can also be concluded from these results that this kind of basal regulation of CpcA-dependent genes is not as significant as under starvation conditions and that small differences in the amounts of some proteins are required to support pathogenicity of this fungus. To find out to what extent $\mathrm{CpcA}$ regulates these genes, further experiments like promoter analysis should be performed. In addition, deletion of target genes of CpcA under non-starvation condition may be a possibility to address the role of $\mathrm{CpcA}$ in pathogenicity. 


\subsection{References}

Carsiotis, M., Jones, R.F., and Wesseling, A.C. (1974) Cross-pathway regulation: histidinemediated control of histidine, tryptophan, and arginine biosynthetic enzymes in Neurospora crassa. J Bacteriol 119: 893-898.

da Silva Ferreira, M.E., Malavazi, I., Savoldi, M., Brakhage, A.A., Goldman, M.H., Kim, H.S., Nierman, W.C., and Goldman, G.H. (2006) Transcriptome analysis of Aspergillus fumigatus exposed to voriconazole. Curr Genet 50: 32-44.

Davey, M.J., Jeruzalmi, D., Kuriyan, J. and O’Donnell, M. (2002) Motors and switches: AAA+ machines within the replisome. Nature Rev Mol Cell Biol 3: 826-835.

Davis, R.H. (2000) Neurospora: Contributions of a Model Organism. Oxford, England: Oxford University Press.

Deng, J., Lu, P.D., Zhang, Y., Scheuner, D., Kaufman, R.J. (2004) Translational repression mediates activation of nuclear factor kappa B by phosphorylated translation initiation factor 2. Mol Cell Biol 24: 10161-10168.

Fernandez-Arenas, E., Cabezon, V., Bermejo, C., Arroyo, J., Nombela, C., Diez-Orejas, R., and Gil, C. (2007) Integrated proteomics and genomics strategies bring new insight into Candida albicans response upon macrophage interaction. Mol Cell Proteomics 6: 460-478.

Hannig, E.M., and Hinnebusch, A.G. (1988) Molecular analysis of GCN3, a translational activator of GCN4: evidence for posttranslational control of GCN3 regulatory function. Mol Cell Biol 8: 4808-4820.

Hannig, E.M., Williams, N.P., Wek, R.C., and Hinnebusch, A.G. (1990) The translational activator GCN3 functions downstream from GCN1 and GCN2 in the regulatory pathway that couples GCN4 expression to amino acid availability in Saccharomyces cerevisiae. Genetics 126: 549-562.

Hanson, P.I., and Whiteheart, S.W. (2005) AAA+ proteins: have engine, will work. Nat Rev Mol Cell Biol 6: 519-529.

Hinnebusch, A.G. (1984) Evidence for translational regulation of the activator of general amino acid control in yeast. Proc Natl Acad Sci U S A 81: 6442-6446.

Hinnebusch, A.G. (2005) Translational regulation of GCN4 and the general amino acid control of yeast. Annu Rev Microbiol 59: 407-450.

Hoffmann, B., Valerius, O., Andermann, M., and Braus, G.H. (2001) Transcriptional autoregulation and inhibition of mRNA translation of amino acid regulator gene $c p c A$ of filamentous fungus Aspergillus nidulans. Mol Biol Cell 12: 2846-2857.

Krappmann, S., Bignell, E.M., Reichard, U., Rogers, T., Haynes, K., and Braus, G.H. (2004) The Aspergillus fumigatus transcriptional activator CpcA contributes significantly to the virulence of this fungal pathogen. Mol Microbiol 52: 785-799.

Krappmann, S., Bayram, O., and Braus, G.H. (2005) Deletion and allelic exchange of the Aspergillus fumigatus veA locus via a novel recyclable marker module. Eukaryot Cell 4: 1298-1307.

Natarajan, K., Meyer, M.R., Jackson, B.M., Slade, D., Roberts, C., Hinnebusch, A.G., and Marton, M.J. (2001) Transcriptional profiling shows that Gen4p is a master regulator of gene expression during amino acid starvation in yeast. Mol Cell Biol 21: 43474368.

Piotrowska, M. (1980) Cross-Pathway regulation of ornithine carbamoyltransferase synthesis in Aspergillus nidulans. J Gen Microbiol 116: 335-339.

Rolfes, R.J., and Hinnebusch, A.G. (1993) Translation of the yeast transcriptional activator GCN4 is stimulated by purine limitation: implications for activation of the protein kinase GCN2. Mol Cell Biol 13: 5099-5111. 
Sachs, M.S. (1996) General and cross-pathway controls of amino acid biosynthesis. In: The Mycota: Biochemistry and Molecular Biology, ed. R. Brambl and G.A., Marzluf Heidelberg, Germany: Springer Verlag III: 315-345.

Sasse, C., Bignell, E.M., Hasenberg, M., Haynes, K., Gunzer, M., Braus, G.H., and Krappmann, S. (2008) Basal expression of the Aspergillus fumigatus transcriptional activator CpcA is sufficient to support pulmonary aspergillosis. Fungal Genet Biol

Tian, C., Kasuga, T., Sachs, M.S., and Glass, N.L. (2007) Transcriptional Profiling of Cross Pathway Control in Neurospora crassa and Comparative Analysis of the Gen4 and CPC1 Regulons. Eukaryot Cell 6: 1018-1029.

Wang, Q., Song, C., and Li, C.C. (2004) Molecular perspectives on p97-VCP: progress in understanding its structure and diverse biological functions. J Struct Biol 146: 44-57.

Yang, R., Wek, S.A., and Wek, R.C. (2000) Glucose limitation induces GCN4 translation by activation of Gen2 protein kinase. Mol Cell Biol 20: 2706-2717. 


\section{Curriculum Vitae}

Christoph Sasse

Born on November the $13^{\text {th }}$, 1977 in Hess. Lichtenau, Germany

\section{Education:}

1984-1987 Primary Education in Sontra

1987-1990 Primary Education in Wolfenbüttel

1990-1997 Secondary Education at the Theodor-Heuss-Gymnasium in Wolfenbüttel

\section{Scientific Background:}

1999-2004 Study of biology at the Georg-August Universität Göttingen, Germany.

Graduation in biology with the diploma thesis "Etablierung der ChromatinImmunopräzipitations-Technik in dem Hyphenpilz Aspergillus fumigatus" in the Institut für Mikrobiologie und Genetik, Abteilung für Molekulare Mikrobiologie und Genetic of Prof. Dr. G.H. Braus.

2005-2008 Scientific assistant at the Georg-August Universität Göttingen (Germany), in the Institut für Mikrobiologie und Genetik, Abteilung für Molekulare Mikrobiologie und Genetik of Prof. Dr. G.H. Braus. 